\title{
Evolution of amide bond formation
}

\author{
Madeleine M. Joullié and Kenneth M. Lassen \\ Department of Chemistry, University of Pennsylvania, 231 South 34th Street, Philadelphia, \\ Pennsylvania 19104, U. S. A. \\ E-mail: mjoullie@sas.upenn.edu
}

This paper is dedicated to Drs Bruce E. Maryanoff and Cynthia A. Maryanoff for their outstanding contribution to organic chemistry as industrial chemists

DOI: $\underline{\text { http://dx.doi.org/10.3998/ark.5550190.0011.816 }}$

\begin{abstract}
The presence of carboxamide groups is found in a large array of biologically important compounds. Peptides and proteins play an important role in modern biology. A key step in peptide production is the formation of the peptide bond, which involves amide bond formation. Chemical synthesis of large peptides by intermolecular coupling of smaller peptides using conventional peptide bond-forming techniques did not realize its potential advantages until recently. Using an approach based on highly specific and efficient chemical ligation of two peptide segments, the two subunits were ligated to form a new peptide bond. Native chemical ligation extended the concept by creating a native peptide bond and providing new approaches to protein engineering.
\end{abstract}

Keywords: Amide bond formation, coupling reagent, peptide, chemical ligation, native chemical ligation

\section{Table of Contents}

Introduction

1. Amide Bond Formation

1.1. Approaches to amide bond formation

1.2. Racemization

2. Coupling Additives

3. Common Coupling Reagents

3.1. Azides

3.2. Active esters 
3.3. Acyl halides

3.4. Anhydrides

3.5. N-Carboxy- $\alpha$-aminoacid anhydrides (Leuchs' anhydrides)

3.6. Carbodiimides

3.7. Pyrocarbonates

3.8. Isoxazolium salts

3.9. Phosphonium and phosphonic salts

3.10. Immonium salts

3.11. Aminium salts

4. Other Coupling Reagents

4.1. Organophosphorus esters

4.2. Activated Phosphate

4.3. Triazoles

4.4. Triazines

4.5. Thiazolium Salts

4.6. Imidazolium and benzimidazolium salts

4.7. Thiouronium salts

4.8. (Chlorophenylthiomethylene)dimethylammonium chloride (CPMA)

4.9. 2,4-Bis(ethylsulfonyl)-1-napthol (BMSN)

4.10. Bis-[ $\alpha, \alpha$-(trifluoromethyl)benzyloxy]diphenylsulfur(IV)

4.11. Tantalum pentachloride

4.12. N-Alkylhydroxylamines and $\alpha$-keto acids

5. Chemical Ligation

5.1. Native chemical ligation (NCL) approaches

6. Discussion

7. Conclusions

8. Acknowledgements

9. References

\section{Introduction}

The presence of carboxamide groups is found in a large array of biologically important compounds. Peptides and proteins play an important role in modern biology. A key step in peptide production is the formation of the peptide bond, which involves amide bond formation. ${ }^{1}$ The process usually requires activation of a carboxylic acid moiety in the presence of coupling reagents. Activation consists of the replacement of the hydroxyl group of the carboxylic acid with a leaving group as the acid would otherwise simply form salts with the amine. The reaction of the activated intermediate and the amine is known as the coupling reaction and the activators are coupling reagents. ${ }^{2}$ The synthesis of peptides depends on the combination of twenty 
proteinogenic amino acids and a growing number of non-coded amino acids, thereby requiring efficient coupling reagents. Although new coupling reagents are always important, the success or failure of the coupling may not be ascribed solely to the reagent but rather to a mechanism that may be appropriate for one substrate but not for another depending on their stereochemistry and conformation of the precursors. Therefore, understanding the requirements of the substrate and the basic mechanisms of different coupling reagents are more useful to the success of a coupling reaction than the reactivity of the coupling reagent. It is this approach that led to the evolution of amide bond formation.

\section{Amide Bond Formation}

\subsection{Approaches to amide bond formation ${ }^{3}$}

There are three different ways of forming amide bonds although they are not always distinguishable: (1) the reactive acylating agent is formed in a separate step, prior to the reaction with the amine; (2) the acylating reagent is isolated and purified before the reaction; and (3) the reagent is generated in situ in the presence of the amino group by adding the activating agent to a mixture of the two reagents.

\subsection{Racemization 4}

A common problem in peptide synthesis is the racemization of the amino acid, usually through 5(4H)-oxazolone (azlactone) formation and other side reactions. Understanding the mechanisms of racemization is important for their prevention. Three pathways are recognized: elimination of the alpha-proton; racemization via reversible beta-elimination and racemization through azlactones. The most important mechanism of racemization involves the formation of azlactones, which in turn depends on the ease of removal of the most acidic proton abstracted by bases from the chiral center (Figure 1). ${ }^{5}$ Resonance stabilization of the carbanion generated in the process drives the reaction to the right and the stability of the anion is enhanced by electron-withdrawing effects of the acyl group. The influence of the $\mathrm{N}$-acyl group on the stability of the anion can range from stabilization (formyl and trifluoroacetyl groups) to destabilization (benzyloxycarbonyl, tert-butoxycarbonyl and other alkoxycarbonyl groups). Destabilization is due to the electron release effect of these groups. The role of the base is also important as it abstracts the most acidic proton. 

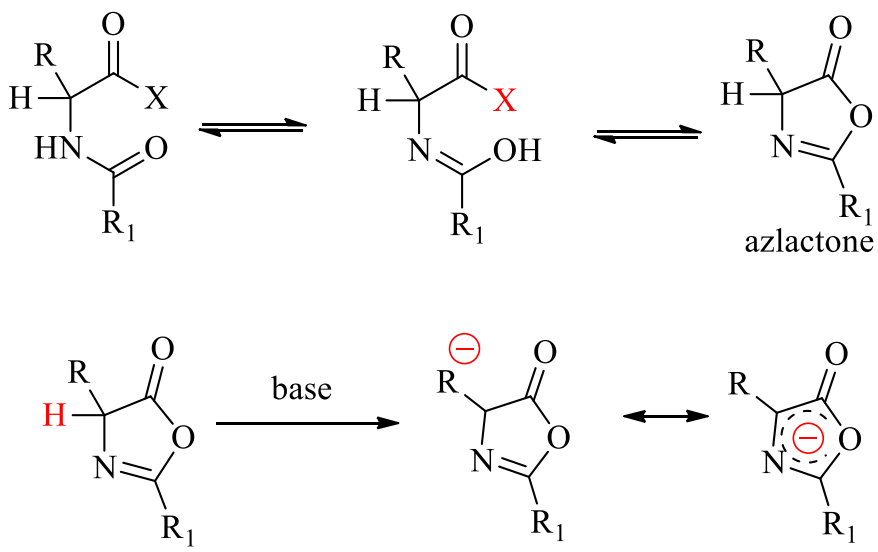

Figure 1. Formation of azlactones.

\section{Coupling Additives}

Ideally, peptide bond formation should be fast, quantitative and carried out under mild conditions without affecting adjacent stereogenic centers, without side reactions and with easily removable side products. Although much progress has been achieved, some problems still remain. A recent comprehensive review of all coupling reagents and their acronyms is available. ${ }^{1}$

While the cyclization of homo- or heterodetic peptides is not addressed directly in this report, it is an important application of coupling reagents. In such cases, it should be remembered that intramolecular cyclizations are best carried out in highly diluted media $\left(10^{-3}\right.$ to $\left.10^{-4} \mathrm{M}\right)$ and as a result the coupling reaction will be slow. Therefore, the activated intermediate should not undergo unimolecular or solvent-induced decomposition. The amino group is best protected as a carbamate and special conditions may be needed for sterically hindered and N-methylated amino acids. Tetramethyluronium derivatives have been reported to be used in end-capping the amino group involved in the macrocyclization. ${ }^{6}$

Additives are used in coupling reactions to inhibit side reactions and reduce racemization. The rate of racemization of an alpha-amino acid derivative depends on temperature, reaction conditions and its substituents. A considerable increase in ease of racemization is also due to the activating acyl substituent. An increase in acylating efficiency (measured by the rate constant) is achieved by thermodynamic activation of the acyl function and this activation increases the vulnerability to racemization. Epimerization occurs as an activated acyl derivative is formed or when it is reacted with the amino function. ${ }^{7}$

The reactivity of peptide coupling reagents may be enhanced by the addition of additives that may also serve to reduce the extent of racemization. For instance, active species can be captured

using a hydroxylamine derivative to give the corresponding active ester. Many additives have a $\mathrm{X}-\mathrm{OH}$ structure that can form active esters with the carboxylic acid. 
NOTE: It should be noted at this point that the coupling reagents field is replete of acronyms. ${ }^{1}$ While their use is unavoidable, practitioners should try to visualize the structure rather than using acronyms.

Several common additives are: $N$-hydroxysuccinimide (HOSu), $N$-hydroxy-5-norbornene2,3-dicarboximide (HONB), 1-hydroxybenzotriazole (HOBt), 6-chloro-1-hydroxybenzotriazole (6-Cl-HOBt), 1-hydroxy-7-azabenzotriazole (HOAt) and more recently 3-hydroxy-4-oxo-3,4dihydro-1,2,3-benzotriazine (HODhbt) and its aza derivative (HODhat) (Figure 2).<smiles>O=C1CCC(=O)N1O</smiles>

$\mathrm{HOSu}$<smiles>O=C1C2C3C=CC(C3)C2C(=O)N1O</smiles>

HONB<smiles>[X]c1ccc2nnn(O)c2c1</smiles>

HOBt $\quad \mathrm{X}=\mathrm{H}$

Cl-HOBt $\mathrm{X}=\mathrm{Cl}$<smiles>On1nnc2cccnc21</smiles>

HOAt<smiles>O=c1c2ccccc2nnn1O</smiles>

HODhbt<smiles>O=c1c2ncccc2nnn1O</smiles>

HODhat<smiles>O=c1c2ncccc2ncn1O</smiles>

HODhad<smiles>On1nncc1Cl</smiles>

HOCt<smiles>CCOC(=O)c1cn(O)nn1</smiles>

HOEt<smiles>On1nnc2ccc(-c3ccccc3)cc21</smiles>

Polymer-supported additives

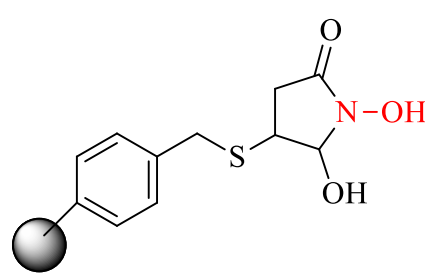

Figure 2. Common coupling additives.

Additives are also useful in solid-phase peptide synthesis (SPPS), and polymer-supported additives are available. The originally proposed additive, $N$-hydroxysuccinimide was tested in solid-phase synthesis. Polymer-supported $N$-hydroxysuccinimide (P-HOSu) has been prepared from a styrene/maleic anhydride co-polymer and used as an economically, simple, racemizationreducing additive for the peptide coupling of amino acid derivatives. The polymeric reagent promotes acylation, facilitates purification and is recovered by simple filtration. ${ }^{8} \mathrm{~A}$ more rigid structurally related additive, $N$-hydroxy-5-norbornene-2,3-dicarboximide (HONB) was reported to prevent side reactions but was not tested in solid-phase synthesis.

Systematic investigations by König and Geiger led to the introduction of 1hydroxybenzotriazole (HOBt) as an additive to dicyclohexylcarbodiimide (DCC) to reduce racemization. ${ }^{9}$ The active species in solution is the 1-hydroxybenzotriazole ester that undergoes aminolysis at a rate about $10^{3}$-fold faster than the ester formed from HOSu. DMF is always present to solubilize HOBt and it is often the exclusive solvent. Some investigators routinely add HOBt to all DCC-mediated couplings. The active species may be regarded as mixed anhydrides of a protected amino acid and a hydroxamic acid (Scheme 1). 


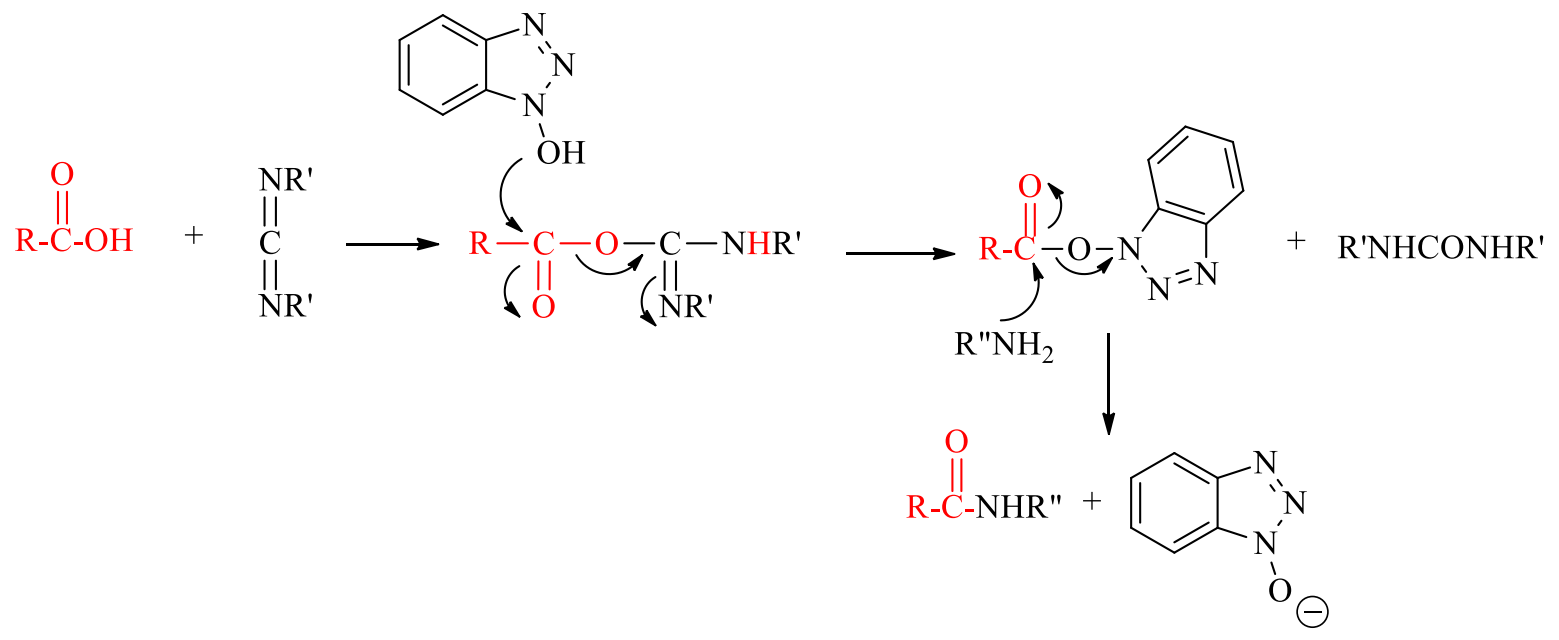

Scheme 1. Formation of 1-hydroxybenzotriazole ester.

Since the acceptance of HOBt, other benzotriazole derivatives were proposed such as 1 hydroxy-6-chlorobenzotriazole (Cl-HOBt) and 1-hydroxy-7-azabenzotriazole (HOAt). is reported to be a more efficient additive that speeds up the coupling process, reduces racemization, and provides a visual indication (yellow to colorless) of the reaction end point. It incorporates in a single molecule a 1:1 mixture of HOBt and a tertiary amine, which is a better catalyst than HOBt. A noticeable reactivity enhancement occurs. A possible reason for the enhancement is shown in Figure 3. For example, acylation of a hindered amine with 2phenylpropanoic acid was achieved in 22 hours in the presence of HOAt whereas HOBt provided only a trace of product. With HOAt the amide was formed within 30-50 minutes in $87 \%$ yield, whereas with $\mathrm{HOBt}$ the reaction was incomplete after 7.5 hours. ${ }^{10}$

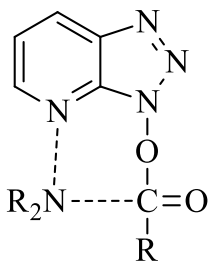

Figure 3. Enhanced reactivity due to 1-hydroxy-7-azabenzotriazole (HOAt).

The use of Cl-HOBt represents a good compromise between HOAt and HOBt in terms of reactivity and price. ${ }^{11}$ The esters of the aza derivative of HODhbt, HODhat, are reported to be more reactive than those of HOAt, which are considered the most reactive among these esters. Addition of benzotriazoles to carbodiimide-based coupling reagents form benzotriazole active esters that are less reactive than the O-acylisoureas formed from carbodiimides, thereby reducing racemization of the protected amino acid and avoiding the formation of undesired derivatives (Figure 4). 


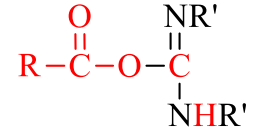

$O$-acylisourea

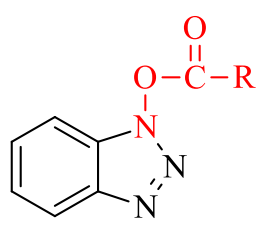

benzotriazole active ester

Figure 4. Active esters from carbodiimides and benzotriazoles.

6-Chloro-1-hydroxybenzotriazole is as reactive as 1-hydroxybenzotriazole but is more acidic $\left(\mathrm{pK}_{\mathrm{a}} 3.35\right.$ compared to $\left.\mathrm{pK}_{\mathrm{a}} 4.30\right)$ and therefore affords a better leaving group. Its active esters are more reactive than HOBt esters. The chlorine atom stabilizes the structure and makes it a less hazardous reagent. HOBt and HOAt are less stable and therefore more difficult to transport and store. The same additives may also be used for aminium salt-mediated coupling reactions, for instance with HBTU.

Although HODhat (3-hydroxy-4-oxo-3,4-dihydro-5-azabenzo-1,2,3-triazene) and HODhad (3-hydroxy-4-oxo-3,4-dihydro-5-azabenzo-1,3-diazene) are structurally similar, the reactivities of their esters are very different, which demonstrates the neighboring group effect of the nitrogen atom in the adjacent pyridine ring. Comparison between the esters of HOBt and HODhad shows the importance of the carbonyl group. In the case of benzylamine all reactions were rapid whereas for the more hindered $\mathrm{N}$-methyl derivatives, clear reactivity differences were noted and the reactivity order was: HODhat $>$ HOAt $>$ HODhbt $>$ HODhad $>$ HOBt. ${ }^{12}$ Coupling reagents degrade rapidly in the absence of a carboxylic acid function. Under these conditions aza derivatives are more labile than benzotriazole derivatives.

Although 1-hydroxybenzotriazole and its derivatives are widely used as peptide coupling reagents, researchers should be aware that such compounds show explosive properties when heated under closed vessels or when subjected to mechanical stimulus. ${ }^{13,14}$

\section{Common Coupling Reagents}

\subsection{Azides}

The azide method is the oldest method used to form peptide bonds. Its permanence is due to the fact that it was believed that protected peptides activated as acid azides could be coupled without racemization. ${ }^{15}$ While not usually racemized, peptide azides can lose optical purity especially in the presence of bases. One of the advantages of the azide method is that azides can be formed directly from esters. The ester is converted to a hydrazide then transformed to an azide with either nitrous acid or an alkyl nitrite (Scheme 2). The procedure obviates the need to remove an ester protecting group. An alternative to hydrazinolysis of esters is the use of protected hydrazides such as benzyloxycarbonylhydrazine whose protecting group can be removed later. 


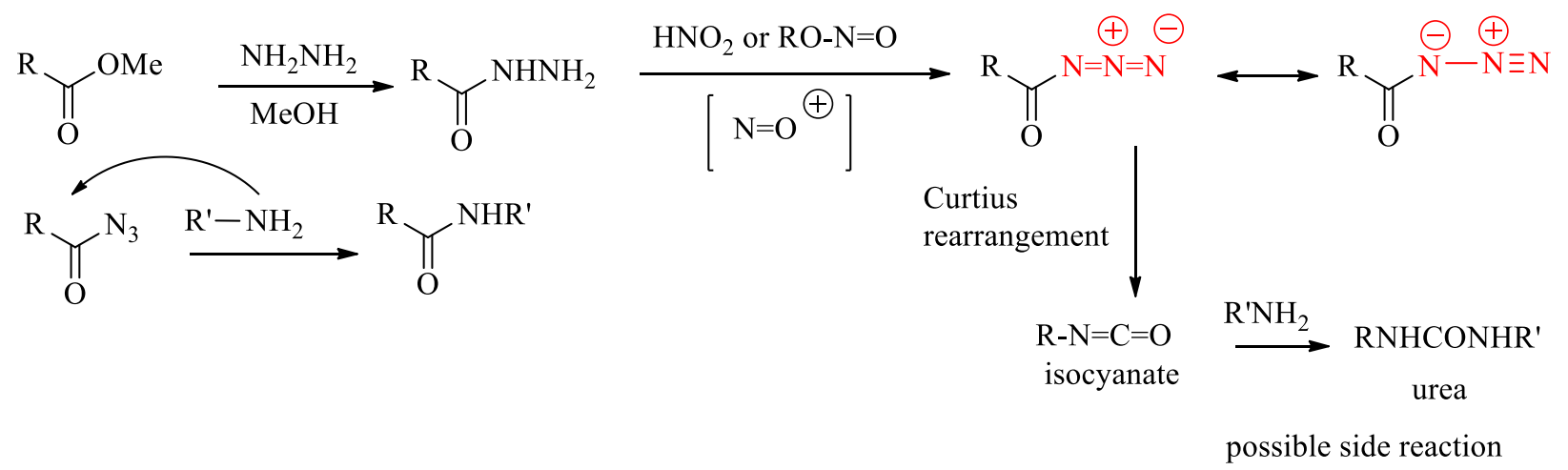

Scheme 2. Formation and reactions of azides.

Peptide azides are not very stable and prone to the Curtius rearrangement (Scheme 4) especially at elevated temperatures. The azide method is less prone to racemization than most other methods. It was suggested, although not proven, that this property might be due to intramolecular general base catalysis by the azide leaving group. Racemization may be avoided by carrying out the reaction at low temperature and high concentration of reactants. Racemization does occur with excess base in polar solvents. Another shortcoming of the process is the slow formation of the peptide bond. Coupling with azides takes considerable time, even several days especially if the reaction is carried out at low temperatures. The reaction is still useful when racemization is a serious problem.

An improved version of the azide method was found by using diphenylphosphoryl azide (DPPA) that can allow the coupling in high yield in the presence of a base in a single operation). ${ }^{16}$ This modified Curtius reaction is much simpler and less laborious than the classical version (Scheme 3).

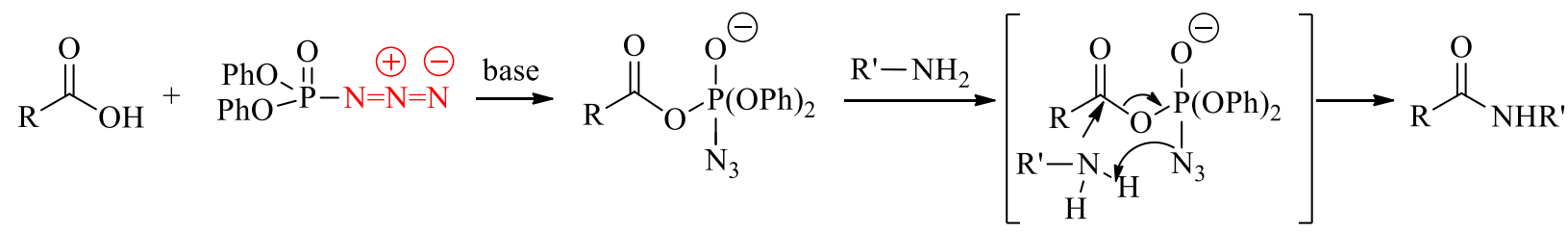

Scheme 3. Coupling with diphenylphosphorylazide.

The most important side reaction in the azide approach is the Curtius rearrangement (Scheme 4) that affords isocyanates, which do not react with their precursors. However under certain conditions azides may react with isocyanates. Photochemically aroyl azides afford aroyl nitrenes that do react with ethyl isocyanate to give 2-aryl-4-ethyl-1,3,4-oxadiazolin-5-ones. ${ }^{17}$ Reaction of alkyl azides with aryl isocyanates provided a convenient method to synthesize 1,4-disubstituted $1 H$-tetrazol-5-(4H)-one (Scheme 5). ${ }^{18}$ 


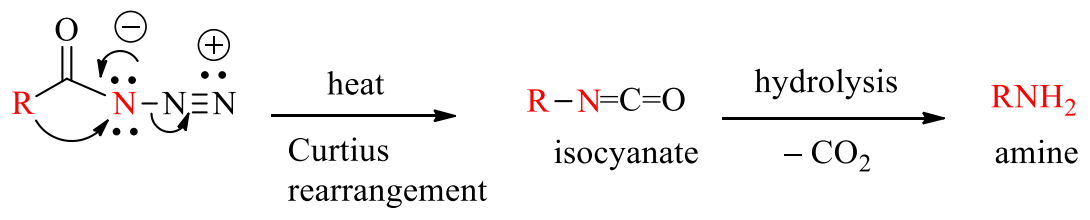

Scheme 4. Curtius rearrangement.

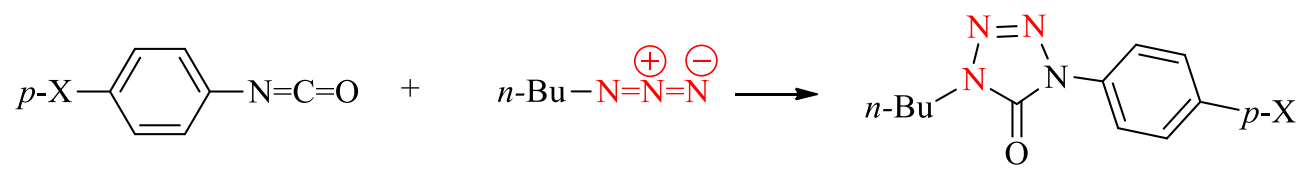

Scheme 5. Formation of 1,4-disubstituted $1 H$-tetrazol-5-(4H)-one.

Reaction of amino groups with isocyanates form urea derivatives. As the bio-isosteric replacement of the amide moiety with a disubstituted urea was shown to afford more potent and efficacious ACAT inhibitors, several ureas were prepared by reaction of amines and isocyanates (Scheme 6). ${ }^{19}$<smiles>[R12]NC(CN)c1ccccc1</smiles>

Scheme 6. Formation of biologically active ureas.

The most important reaction of azides is the Staudinger reaction. The Staudinger reaction involves the attack of trivalent phosphorus on the terminal nitrogen atom of the azide to give the corresponding phosphazide with retention of configuration at the phosphorous atom. The phosphazide undergoes a transformation to an iminophosphorane (aza-ylide) with a loss of nitrogen via a four-membered ring transition state. Hydrolysis of the iminophosphorane affords an amine and triphenylphosphine oxide (Scheme 7).

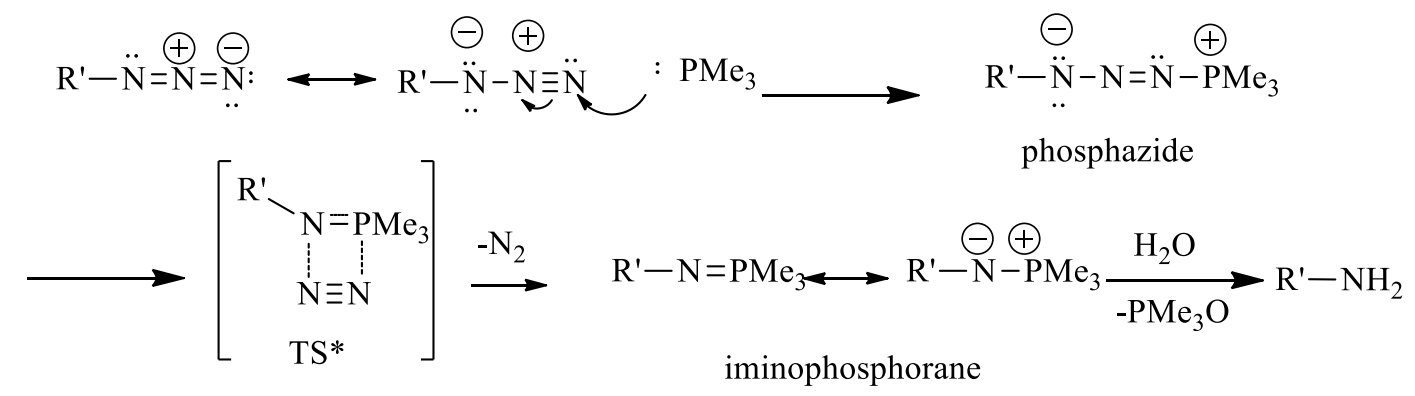

Scheme 7. The Staudinger reaction. 
A general strategy for amide formation involves the Staudinger ligation between a carboxylic phosphinothioester and an azide. ${ }^{20}$ The initial intermediate is an iminophosphorane that rearranges to an amido phosphonium salt which hydrolyses to the amide (Scheme 8). Azides are involved in the synthesis of proteins by native chemical ligation (NCL), the method of choice for the preparation of large peptides and proteins (see later).

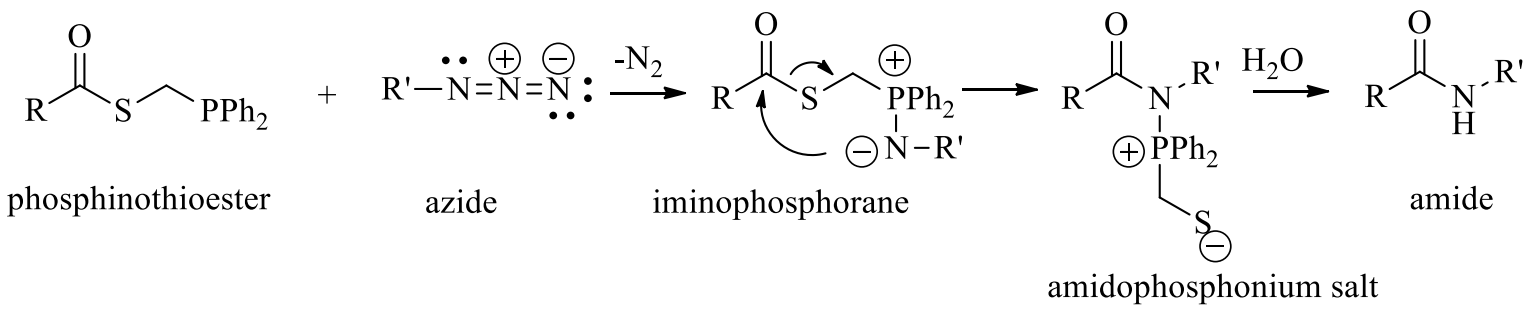

Scheme 8. The Staudinger strategy and formation of the intermediates.

\subsection{Active esters}

Ester aminolysis is the classical method for making amides and was therefore used in the early days of peptide synthesis. It was not realized until the early 1950's that the reaction could be facilitated by modifying the alkoxy leaving group. It was noted that substituted phenyl esters worked better than alkyl ester. The leaving group ability of the aryloxy group is the most important factor. The rate-limiting factor is the collapse of the tetrahedral adduct. Many esters have been investigated. These are often called "active esters." They are often crystalline stable materials and are prepared by DCC-mediated coupling or by mixed anhydride methods. Active esters are at a lower level of activation than some other intermediates previously described and they react selectively with the amine component. Active esters produce fewer side reactions during coupling, including racemization. However, when prepared directly racemization may occur if the carboxyl group is prone to epimerization, especially in the presence of bases. They may be used in the temporary carboxyl blockade, which allows access to protected peptide active esters.

Some active esters are shown in Figure 5. 

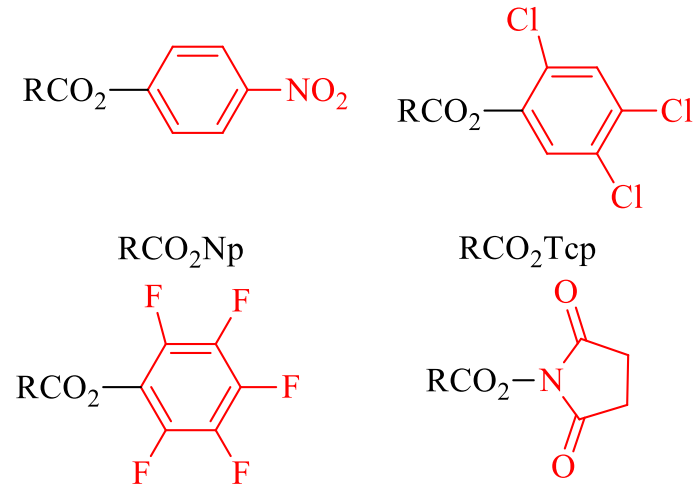

$\mathrm{RCO}_{2} \mathrm{Tfp}$

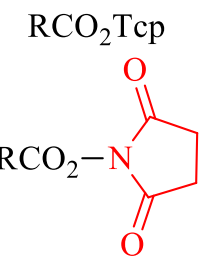

$\mathrm{RCO}_{2} \mathrm{Su}$

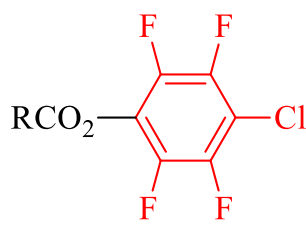

$\mathrm{PfpCl}$<smiles></smiles><smiles>O=P(Oc1c(F)c(F)c(F)c(F)c1F)(c1ccccc1)c1ccccc1</smiles>

FDPP

Figure 5. Some active esters and their abbreviations.

$N$-Hydroxysuccinimide esters are highly reactive and easily purified. They react cleanly and rapidly and the co-product is water-soluble. p-Nitro esters are affordable and crystalline but they are slow to react and the removal of the $p$-nitrophenol may be difficult. Pentachlorophenyl esters are easy to prepare but removal of the pentachlorophenol may be difficult. Pentafluorophenyl esters react within one hour. Active esters can be formed in situ during coupling reactions and are used to improve the purification of coupling reactions. Amides of high purity may be obtained.

A more recent application of active esters is the use of phosphorus esters. Pentafluorophenyl diphenylphosphinate (FDPP) (Figure 5) reported in 1991, can be used directly as a coupling reagent without preactivation. ${ }^{21}$ The use of FDPP for the synthesis of a variety of peptides was compared to other coupling reagents and found to give high yields and good enantiomeric purity except when Fmoc was used as the nitrogen protecting group. ${ }^{22,23}$ Mixed anhydride methods are also useful to prepare active esters (Scheme 9).

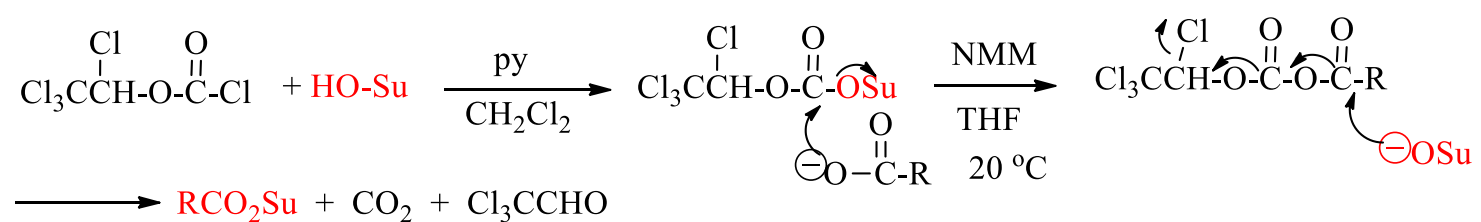

Scheme 9. Mixed anhydride method.

$\mathrm{N}$-Methylmorpholine (NMM) is one of the several tertiary amine bases used in peptide synthesis. Di-isopropylethylamine (DIPEA) or Hünig base and triethylamine (TEA) are also convenient and commonly used tertiary amines.

Active esters, such as the pentafluorophenyl ester have fewer side reactions during coupling (Scheme 10). 


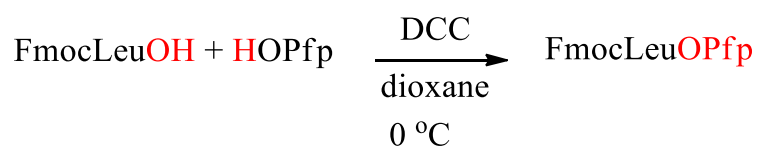

Scheme 10. Formation of active esters.

Active esters may be used in temporary carboxyl blockade, which allows access to protected peptide active esters (Scheme 11).

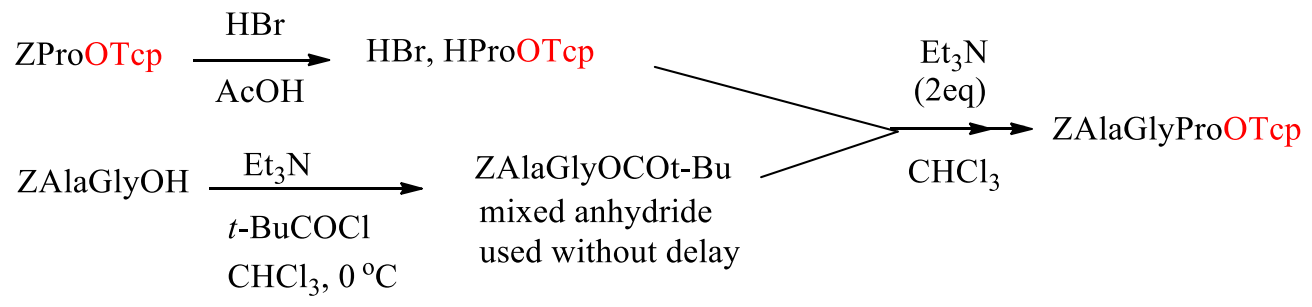

Scheme 11. Acylation of carboxylic acids.

Acylation must be rapid or self-condensation occurs. The reactivities of active esters correlate well with the acidity of the acid related to the ester moiety. Reactivity is important, but ease of removal of the side products is another consideration. For a water-insoluble peptide, a succinimide ester coupling is convenient because $N$-hydroxy succinimide is water-soluble. For a water-soluble product, a halophenyl ester may be best because halophenols are ether-soluble.

Reactive esters are $O$-acyl derivatives of relatively acidic hydroxyl groups. The reactivity of esters of weakly acidic phenols may be increased by providing intramolecular general base catalysis. Thus, monoesters of catechol are more reactive than unsubstituted phenyl esters (Scheme 12).

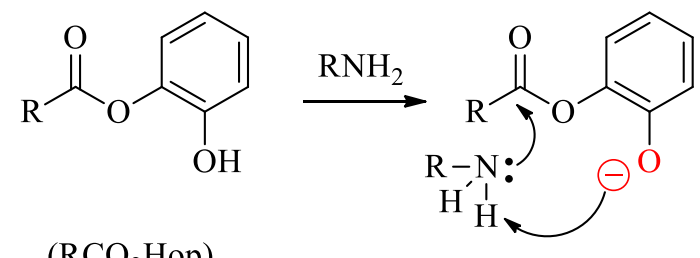

Scheme 12. Activation by intramolecular general base catalysis.

Pentafluorophenyl esters are preformed esters in coupling reactions. A number of pentafluorothiophenyl esters were shown to be useful acyl donors for amide bond formation (Scheme 13). ${ }^{24}$ 


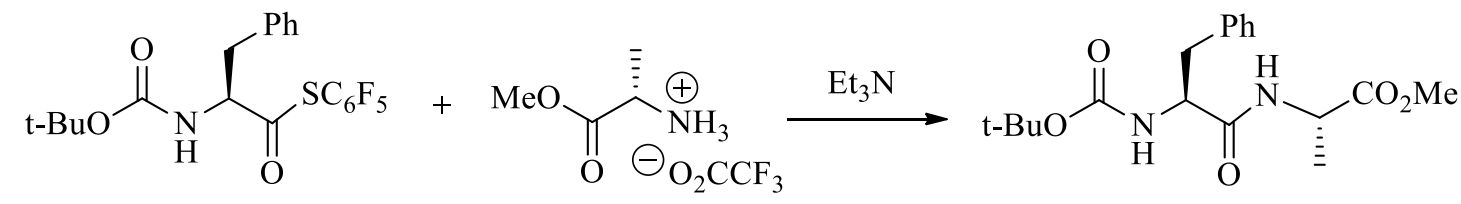

Scheme 13. Coupling with pentafluorophenyl esters.

\subsection{Acyl halides ${ }^{25}$}

The activation of acylamino acids by conversion to the corresponding acid halides is in principle the simplest approach to peptide synthesis but the reagents to prepare acid halides are not compatible with complex or sensitive substrates and simple acylamino acid chlorides cyclize spontaneously to oxazolones and therefore afford racemic peptides. Z-Aminoacid chlorides are isolable but unstable. They decompose to N-carboxy- anhydrides on warming (a) and cyclize under basic conditions to benzyloxyoxazolones (b) (Scheme 14). However, they may be generated in situ by treating the corresponding carboxylic acids with 1-chloro- $N, N, 2$-trimethyl-1propen-1-amine. ${ }^{26}$ Optically active peptides may be isolated after reaction with amino esters but this approach was not utilized until it was found that Fmoc-amino acid chlorides and also fluorides could be prepared easily and are rather stable convenient intermediates.

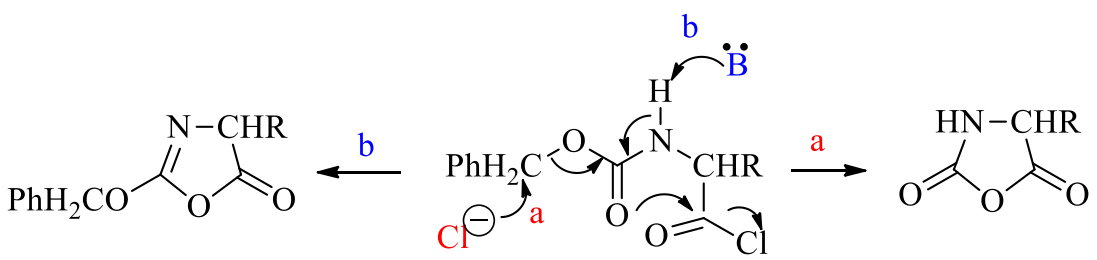

Scheme 14. Reactions of Z-aminoacid chlorides.

Stable Fmoc amino acid chlorides from trifunctional amino acids bearing side-chain protection that incorporates the tert-butyl moiety have been prepared. Cyclic anhydrides often result. As tert-butylfluoroformate was stable as compared to the chloro analog, acid fluorides were examined. Acid fluorides are stable, often crystalline and readily obtained by treating the acid with cyanuric fluoride (Figure 6).<smiles>Fc1nc(F)nc(F)n1</smiles>

cyanuric fluoride $(\mathrm{CFN})_{3}$

Figure 6. Cyanuric fluoride. 
Many sensitive protected Fmoc-amino acids have been prepared (Scheme 15) e.g. $N^{\varepsilon}$ Boc- $N^{\alpha}$-Fmoc-lysine, Fmoc-glutamic acid $\gamma$-tert-butyl ester, $O$-tert-butyl ether of serine using this method. Boc and $\mathrm{Z}$ protection has also been used at lower temperatures (- 30 to $-20{ }^{\circ} \mathrm{C}$ ). At higher temperatures, Boc-Asp and Boc-Glu are contaminated with traces of Leuch's anhydride and Z-Glu with pyroglutamate when activated as an acid fluoride (Scheme 16).

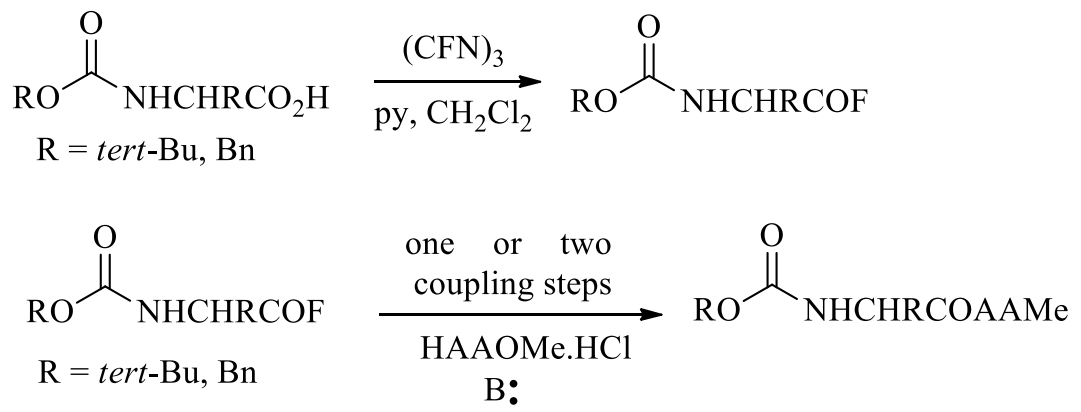

Scheme 15. Formation and use of acid fluorides.

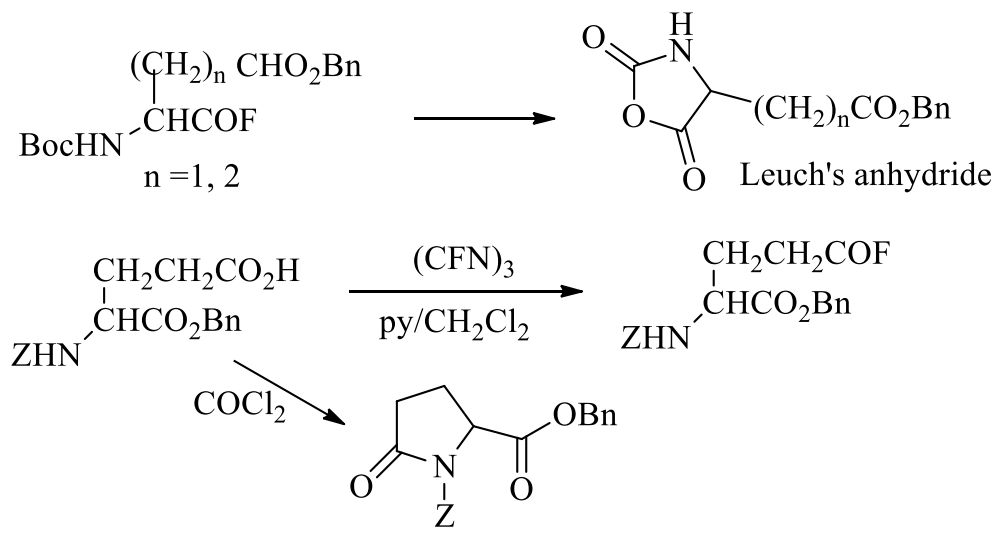

Scheme 16. Side reactions at higher temperature.

The activation of $N, N$-bis Boc or $N$-Boc, $N$-Z amino acids with $\mathrm{SOCl}_{2} / \mathrm{DMF}$ produces cyclic anhydrides. With cyanuric fluoride, acid fluorides are formed. The first examples of fully protected $\mathrm{N}$-acyl derivatives of pyrrole-2-carboxylic acid derivatives (amino acid pyrrolides) were produced in this way (Figure 7). ${ }^{27}$ 


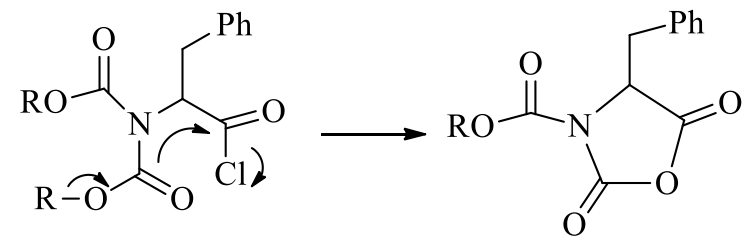

$\mathrm{R}=$ tert-Bu, $\mathrm{Bn}$

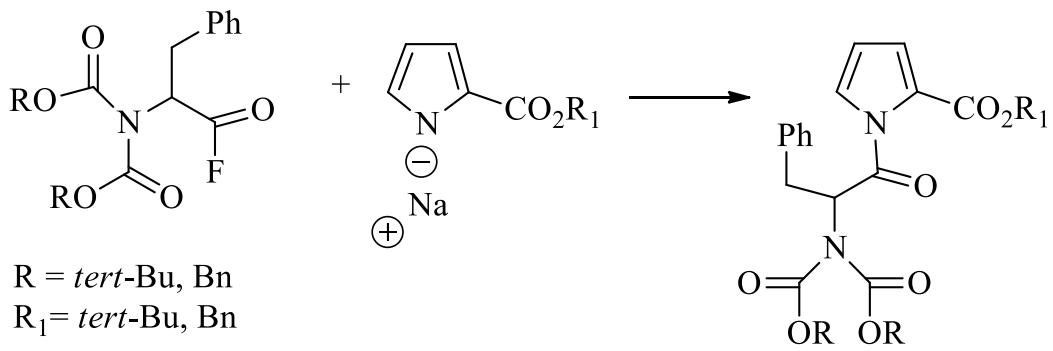

Figure 7. N-Acyl derivatives of pyrrole-2-carboxylic acid derivatives.

The C-F bond is more stable toward neutral nucleophiles $\left(\mathrm{H}_{2} \mathrm{O}\right.$ or $\left.\mathrm{MeOH}\right)$ compared to the $\mathrm{C}-\mathrm{Cl}$ bond, but has similar reactivity towards anionic nucleophiles and amines. Reaction rates are comparable to those of acid chlorides. They are stable to tertiary bases, so no oxazolone formation occurs. No significant loss of chirality occurs in either solution or solid-phase synthesis.

A method of taking advantage of the reactivity of amino acid fluorides without their isolation is to use a new reagent that effects in situ conversion of the acid to the acid fluoride. ${ }^{28}$ Tetramethylfluoroformamidinium hexafluorophosphate (TFFH) is a non-hygroscopic salt stable to handling under ordinary conditions. It is prepared by the reaction of tetramethylchloroformamidinium hexafluorophosphate with excess anhydrous potassium fluoride (Scheme 17).

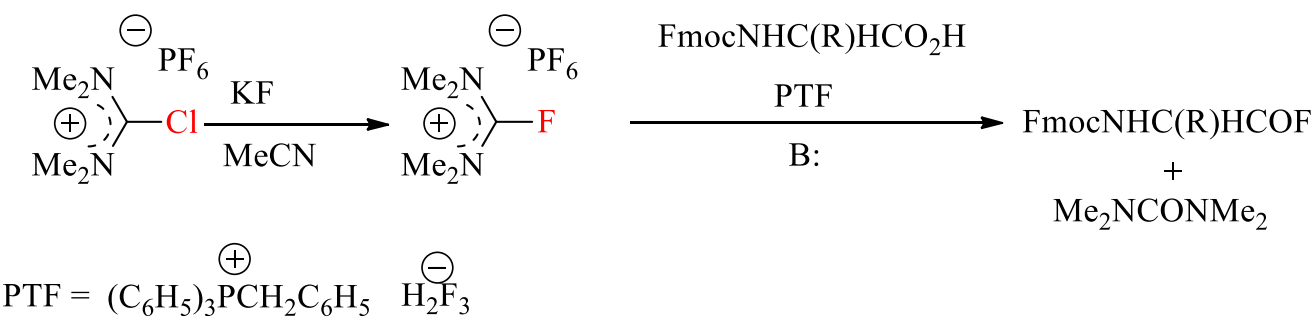

Scheme 17. Preparation and use of TFFH.

The use of TFFH with some amino acids gave results that were less satisfactory than those obtained with isolated amino acid fluorides. This problem was due to inefficient conversion to the acid fluoride, which under these conditions also afforded symmetrical anhydride and oxazolone. The addition of a fluoride additive, benzyltriphenylphosphonium dihydrogen 
trifluoride (PTF) during the activation step avoids the formation of these two undesired products and affords a maximum yield of acid fluoride. ${ }^{29}$

\subsection{Anhydrides}

This term may be applied generally to any activated carboxyl derivative in which the leaving group is the conjugate base of a reasonably strong acid since they result from the condensation of a carboxylic acid component with another acid species minus the elements of water.

When a carboxylic acid is stirred with dicyclohexylcarbodiimide (DCC), dicyclohexylurea is formed immediately along with a symmetrical anhydride. The procedure is clean and rapid. However, it is wasteful of acid, often an expensive protected unit. Mixed anhydrides solve the problem by having only one carbonyl group susceptible to attack by amine. Mixed anhydrides such as pivalic anhydrides are usually generated in situ (Scheme 18).

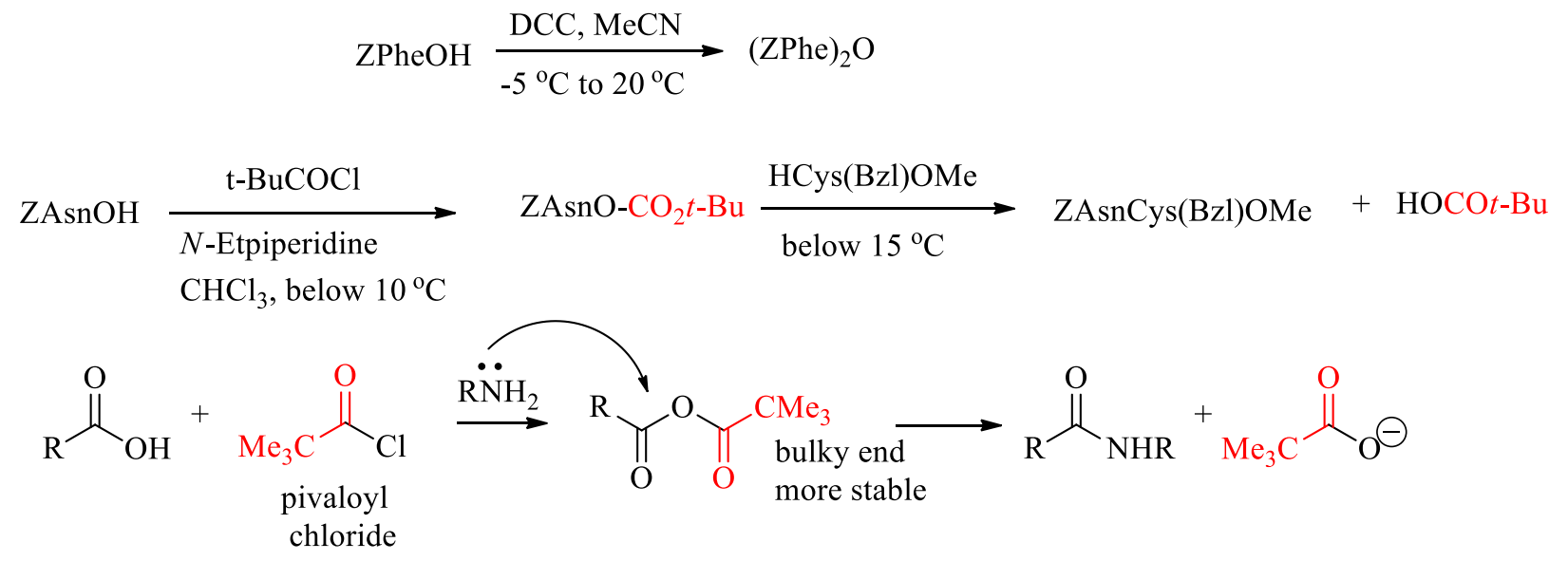

Scheme 18. Mixed anhydrides and their use.

Mixed anhydrides of carbonic acids are also very useful. Although they are transient species, they are isolable and last a few hours. They are used to prepare simple protected peptides in the tripeptide to hexapeptide range that have similar solubility to dicyclohexylurea. Low temperatures and minimal activation times are employed. The fact that two oxygen atoms flank one of the carbonyl group in the activated intermediate diminishes its reactivity so that nucleophilic attack is directed toward the carbonyl of the original carboxyl component (Figure 8). 

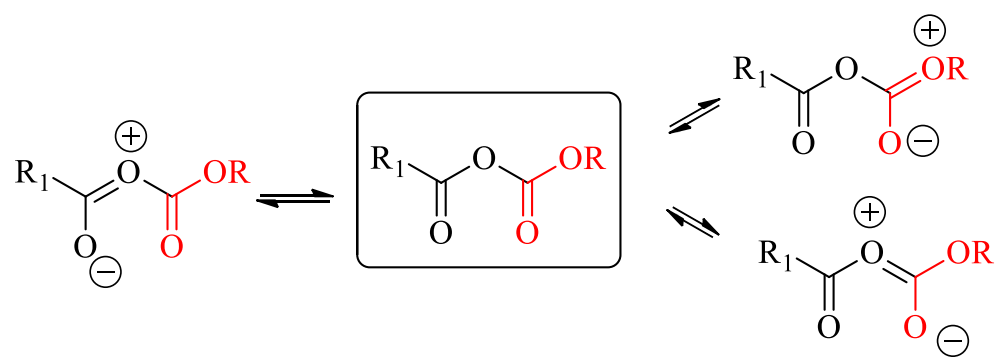

Figure 8. Delocalization in mixed anhydrides.

Alkoxycarbonyl derivatives of the amino group are not usually formed in more than trace amounts except with hindered components. The reaction is usually carried out with one equivalent of a tertiary base in an inert dry solvent at $-10{ }^{\circ} \mathrm{C}$ for a few minutes, followed by addition of the amino component (Scheme 19).

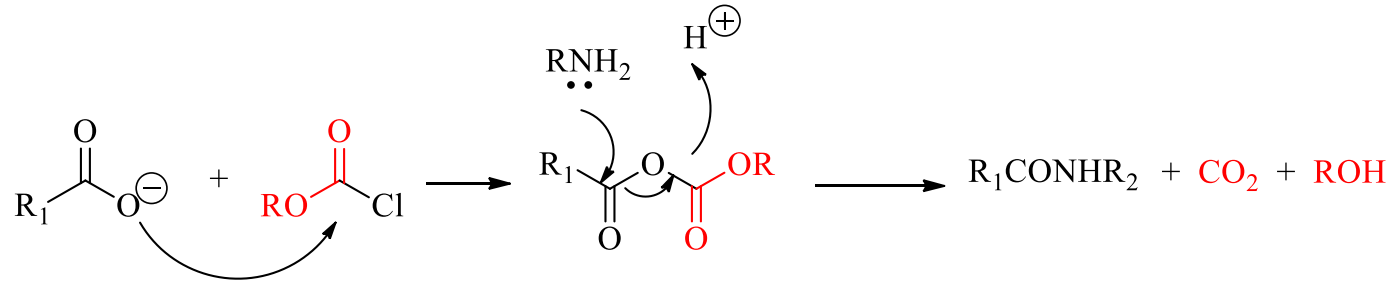

Scheme 19. Reaction with chloroformates.

Ethyl chloroformate or isobutyl chloroformate may be used. Isobutyl chloroformate is the reagent of choice for large-scale syntheses. The use of chloroformates involves separate activation and aminolysis steps but mixed ethyl carbonic anhydrides can also be generated by the reaction of carboxylic acids with 1-ethoxycarbonyl-2-ethoxy-1,2-dihydroquinoline (EEDQ) in the presence of the amino component. This procedure involves direct coupling and because the mixed carbonic anhydride is consumed by aminolysis as soon as it is formed, the side reactions are minimal and workup is simple (Scheme 20). ${ }^{30}$<smiles>[R]OC(=O)[n+]1c(OCC)ccc2ccccc21</smiles>

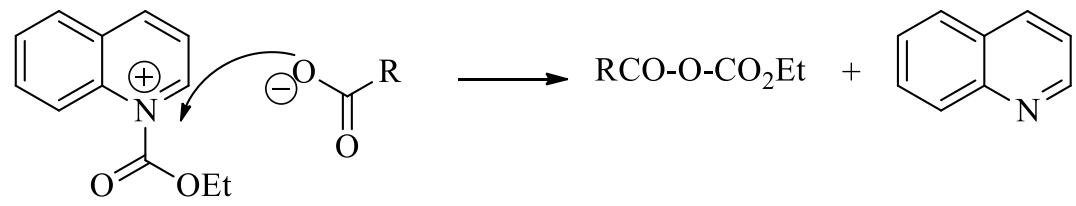

Scheme 20. Generation of mixed carbonic anhydride. 
1-Isobutoxycarbonyl-2-isobutoxy-1,2-dihydroquinoline (IIDQ) is an improved version of EEDQ. Both reagents are easily prepared, easily stored, and not prone to side reactions or racemization (Scheme 21). No tertiary amine bases are needed for these reactions. Since activation of the carboxyl component requires displacement of the alkoxy group from the 2position of the quinoline and intramolecular anhydride formation, practical rates require high concentration of the reactants. However, with properly protected peptides, IIDQ can be used in excess and give satisfactory results.

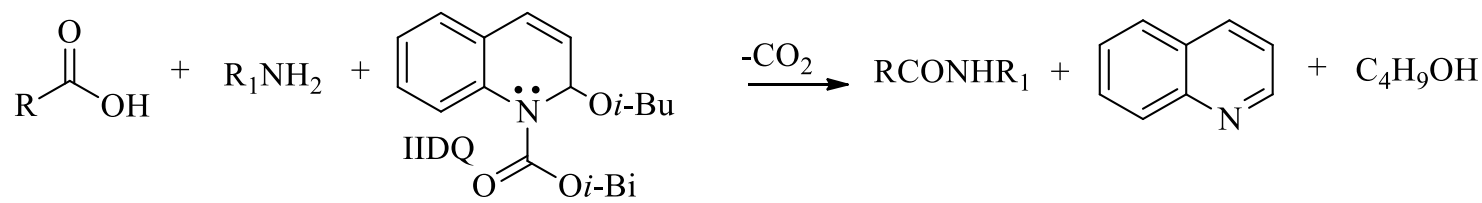

Scheme 21. Reaction with 1-isobutoxycarbonyl-2-isobutoxy-1,2-dihydro quinoline (IIDQ).

A drawback in the use of anhydrides is a side reaction that leads to the formation of a urethane by-product and liberation of the starting amino acid. The reason for urethane formation is the formation of the symmetrical anhydride in the first activation step of the amino acid with the chloroformate during the preparation of the mixed carboxylic-carbonic anhydride intermediate. A solution to the problem is to use an inverse addition of the $\mathrm{N}$-protected amino acid and the base to a solution of the alkyl chloroformate, followed by the addition of the amino component (Scheme 22).

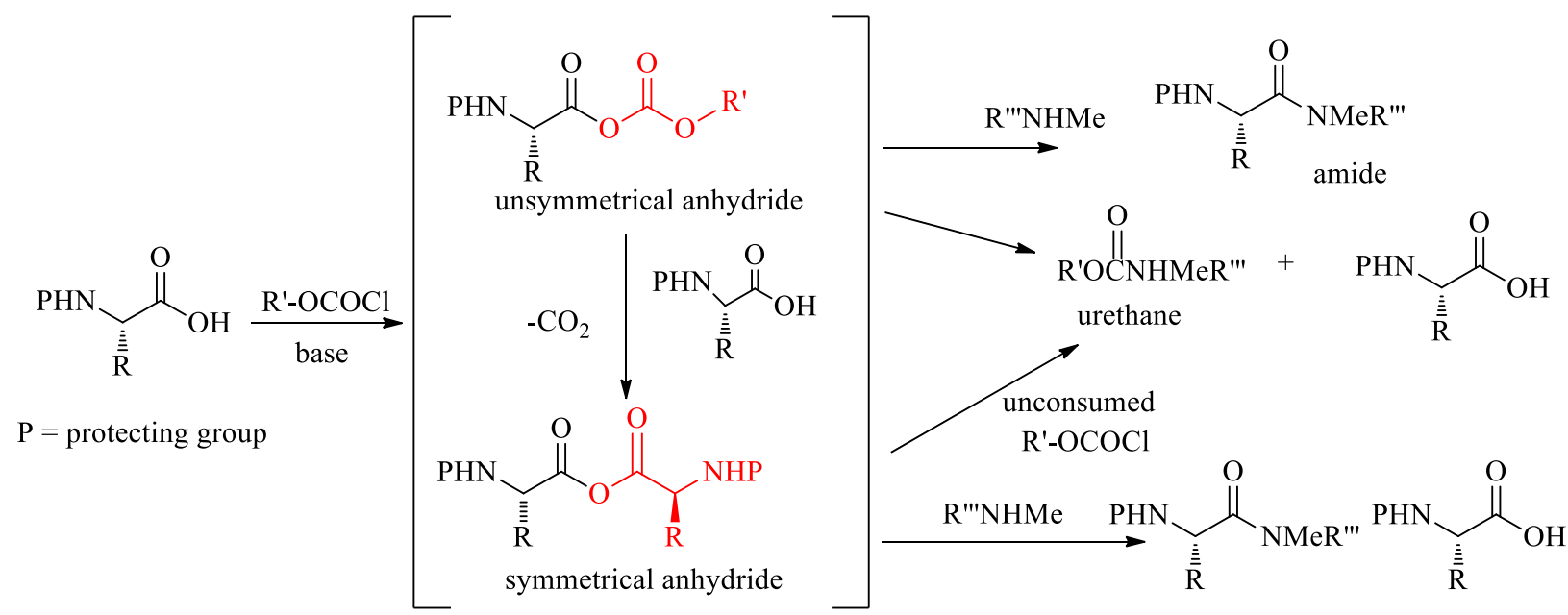

Scheme 22. Synthesis and reactions of mixed anhydrides.

Isopropenyl chloroformate is also an activating agent of $N$-protected amino acids using the mixed anhydride method. ${ }^{31}$ The reagent can be used at room temperature and the products are carbon dioxide and acetone, which are easily removed (Scheme 23). 


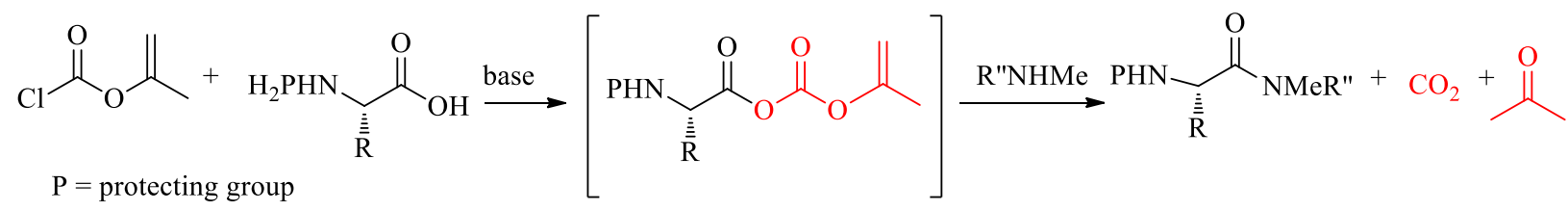

Scheme 23. Reaction with isopropenyl chloroformate.

Phosphite derivatives are another type of mixed anhydride (Scheme 24).

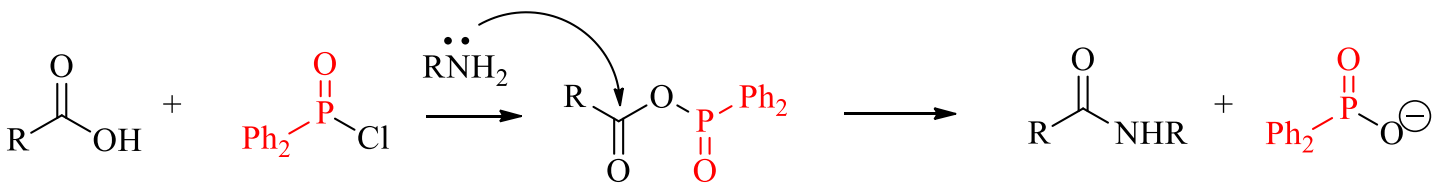

Scheme 24. Diphenylphosphinic mixed anhydrides.

In addition, symmetrical anhydrides may be the actual species in some reactions. Symmetrical anhydrides of Boc, Z, and Fmoc amino acids are generally crystalline. Diphenylphosphinic mixed anhydrides are more regioselective than the mixed carboxylic and carbonic anhydride methods as aminolysis occurs exclusively at the carbonyl of the activated amino acid even for sterically hindered systems. Coupling is clean and workup is simple (Scheme 25).

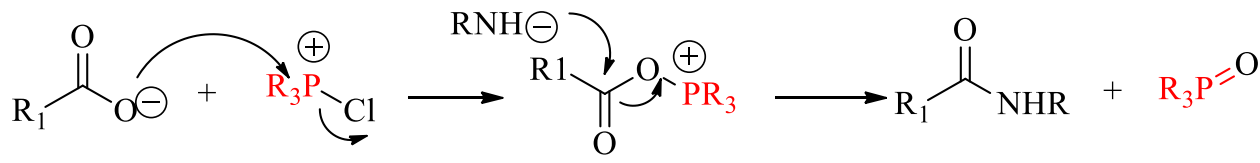

Scheme 25. Coupling with a phosphinic mixed anhydride.

1-Oxo-1-chlorophospholane was found to react with $\alpha$-amino acids to form intermediate mixed phospholanic-carboxylic anhydrides that were stable at $0{ }^{\circ} \mathrm{C}$ (Figure 9 ). The peptides were formed in high yields and were of high quality. The resulting cyclic phosphinic acid was readily removed from solution requiring minimum purification.<smiles>[R]C(=O)OP1(=O)CCCC1</smiles>

Figure 9. Mixed phospholanic-carboxyl anhydride. 
Anhydrides involving diesters of phosphorous acid produce only one acylation product because the second acylation product is reconverted to the mixed anhydride (Scheme 26).

$$
\begin{gathered}
\mathrm{ZNHCHRCO}_{2} \mathrm{P}(\mathrm{OR})_{2}+\mathrm{R}_{1} \mathrm{NH}_{2} \longrightarrow \mathrm{ZNHCHRCONHR}_{1}+(\mathrm{RO})_{2} \mathrm{PNHR}_{1} \\
(\mathrm{RO})_{2} \mathrm{PNHR}_{1}+\mathrm{ZNHCHRCO}_{2} \mathrm{H} \longrightarrow \mathrm{ZNHCHRCO}_{2} \mathrm{P}(\mathrm{OR})_{2}+\mathrm{R}_{1} \mathrm{NH}_{2}
\end{gathered}
$$

Scheme 26. Phosphorous acid diesters.

Another example of an efficient mixed anhydride coupling reagent is a carboxyl-sulfonic mixed anhydride with an $o$-hydroxyarenesulfonyl chloride. ${ }^{32}$ The coupling is a one pot two-step reaction involving a sulfoquinone intermediate, followed by an efficient six-membered acyl transfer reaction to the phenolic carboxyl-sulfonic acid intermediate (Figure 10).
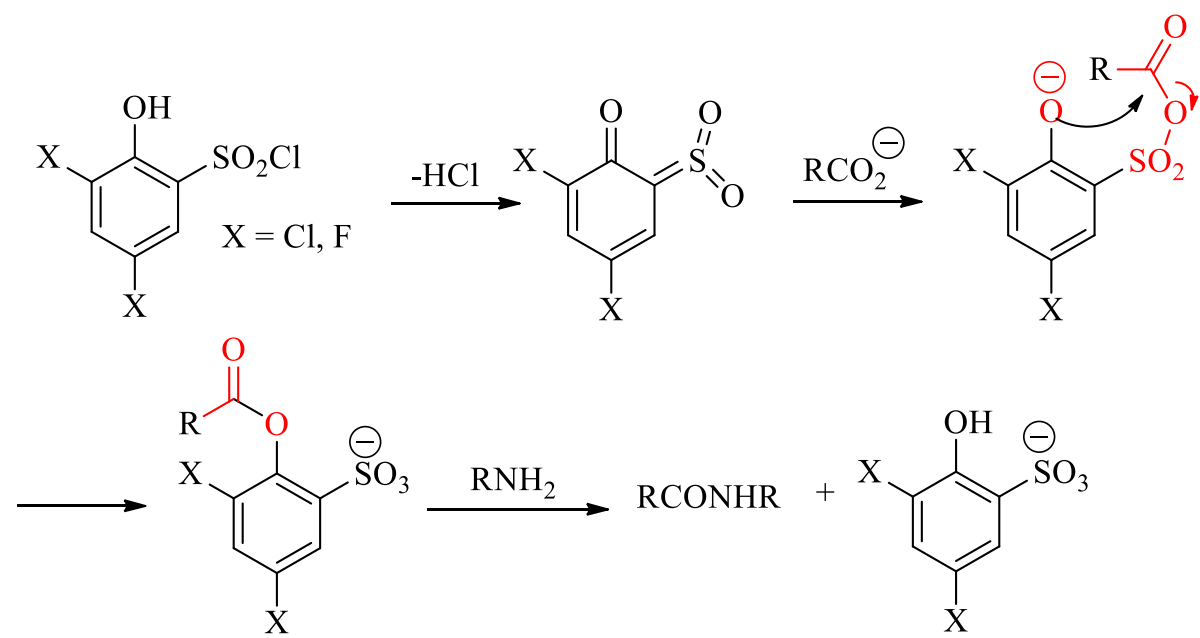

Figure 10. Mechanism for the coupling reaction of a carboxylic-sulfonic anhydride.

Activation of a carboxyl group as a mixed anhydride is also a convenient method to form a peptide bond when $\alpha$-azido esters and trialkylphosphines are used. ${ }^{33}$ Conversion of the carboxyl groups of $\mathrm{N}$-protected $\alpha$-amino acids to the 3,5-dinitrobenzoyl mixed anhydrides, followed by treatment with $\alpha$-azido esters and trialkyl phosphines gave good yields of peptides without appreciable epimerization. The attack of phosphatriazane intermediates to the mixed anhydride is regioselective and the medium essentially neutral. No special additives are needed.

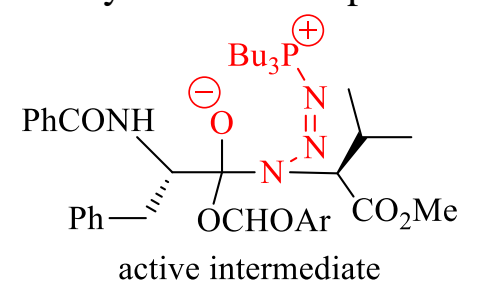

Figure 11. Phosphatriazane attack. 


\subsection{N-Carboxy- $\alpha$-aminoacid anhydrides (Leuchs' anhydrides)}

$\mathrm{N}$-Carboxy- $\alpha$-aminoacid anhydrides (4-alkyloxazolidine-2,5-diones) also known as Leuchs' anhydrides (NCA) (Figure 12) may be obtained by the action of phosgene on $\alpha$-amino acids or the decomposition of alkoxycarbonyl- $\alpha$-amino acid chlorides.

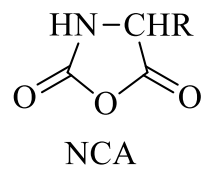

Figure 12. Leuchs' anhydride (NCA).

Treatment of a Leuchs' anhydride with a small amount of a nucleophilic initiator in an organic solvent opens the ring with loss of $\mathrm{CO}_{2}$ to give an amine. The resulting amine attacks another molecule of NCA and so on, forming eventually a homopolyamino acid (Scheme 27).

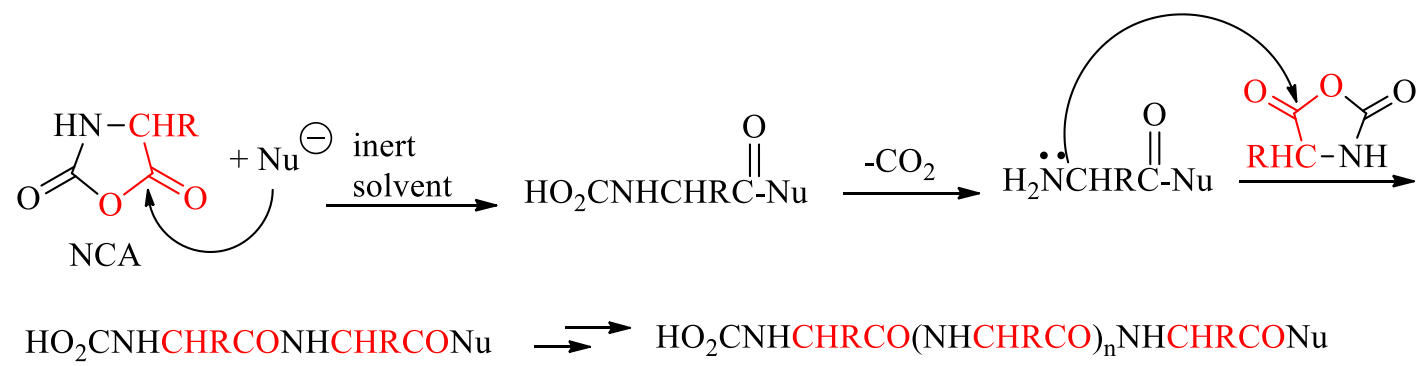

Scheme 27. Formation of homopolyamino acid.

Under carefully controlled conditions NCA can be used for rapid peptide synthesis in aqueous solutions (Scheme 28). However, carbamic acids produced by aminolysis may be unstable and decarboxylation may occur before the aminolysis is completed, giving an amino group that may react with remaining NCA. Therefore the reaction must be monitored.

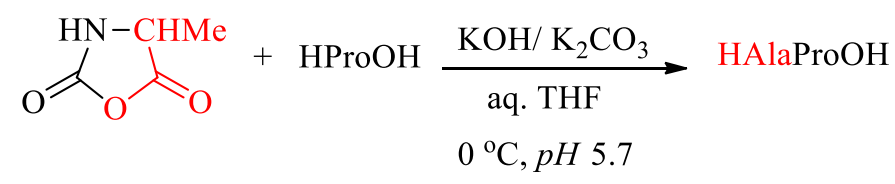

Scheme 28. Peptide formation with NCA.

The thio analogs of NCA (NTA) are preferred for glycine and histidine, but they are prone to racemization. 


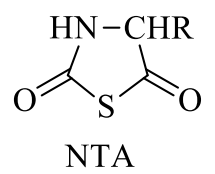

Figure 14. Structure of NTA.

$\mathrm{N}$-Carboxy- $\alpha$-aminoacid anhydrides may be $N$-alkoxycarbonylated in the presence of base to give urethane protected derivatives (UNCA) (Scheme 29). ${ }^{34}$ Fmoc derivatives are crystalline, with good shelf-life properties, that react rapidly with amines to give Fmoc acyl derivatives and carbon dioxide as the only products. Over-reaction cannot occur because deprotection is needed before another residue can be added.
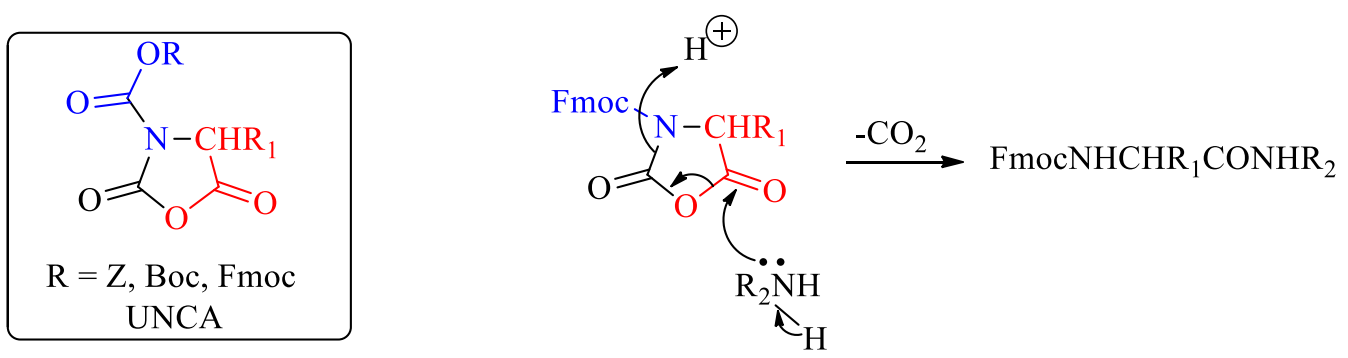

Scheme 29. Reaction of UNCA with amines.

\subsection{Carbodiimides}

Dicyclohexylcarbodiimide has been the single most important reagent for activating carboxyl groups in peptide synthesis for over fifty years. ${ }^{35}$ It has been used to activate symmetrical anhydrides, esters, or as a direct coupling agent. In all cases, the initial step is the addition of the carboxyl group to the carbodiimide function to provide an $O$-acylisourea, one of the most reactive acylating agents. Direct coupling involves mixing equimolar amounts of the amine, the carboxylic acid and dicyclohexylcarbodiimide in an organic solvent. The formation of Oacylisourea is rapid and it subsequently reacts with the amine to form a peptide bond and dicyclohexylurea, which precipitates immediately (Figure 15). 


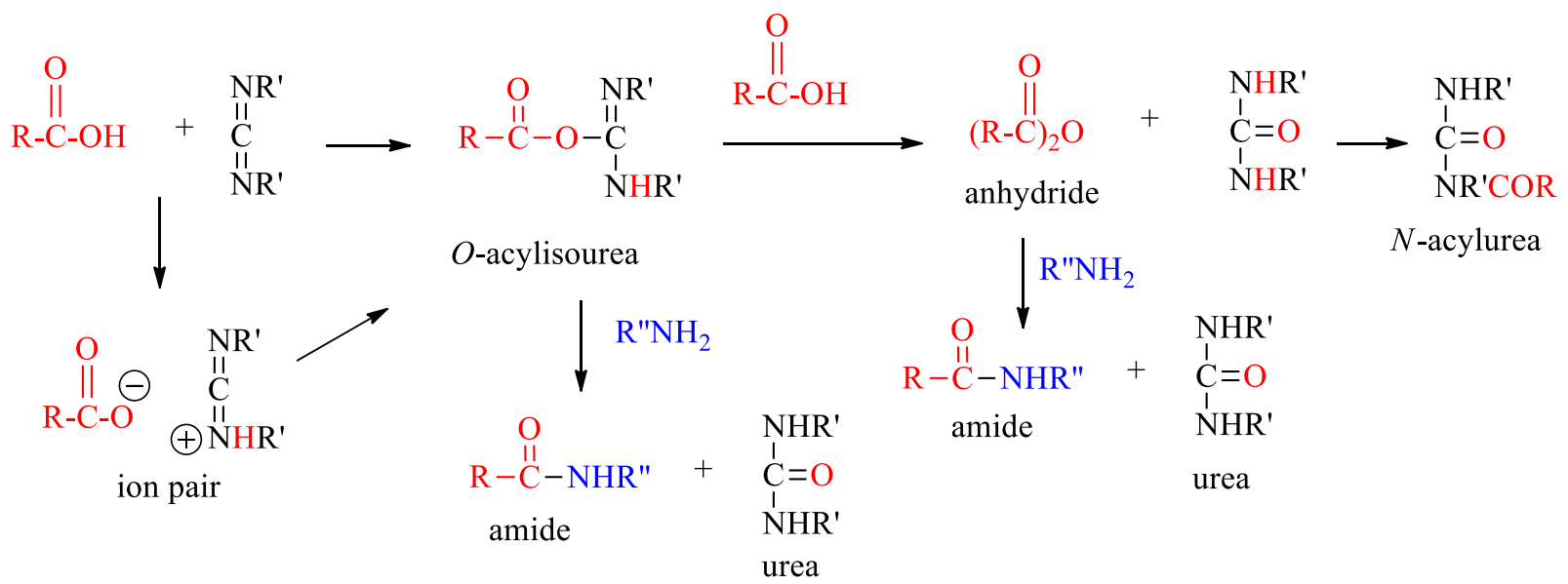

Figure 15. Activation of carboxylic acids by carbodiimides.

Since most dialkylureas are sparingly soluble, their separations are relatively straightforward. The intermediates are highly reactive and side reactions may intervene, especially if the reaction with the amine is delayed. Collapse of the O-acylisourea by intramolecular acyl transfer competes with the attack by nucleophiles. The reaction between urea and the symmetrical anhydride forms an $\mathrm{N}$-acylurea, which reduces the yield and makes purification difficult. The rearrangement occurs rapidly when DMF is used as the solvent (Figure 16).

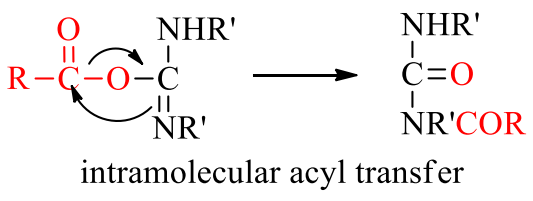

Figure 16. Intramolecular acyl transfer.

The mechanism of carbodiimide-mediated activation is more complex than it appears and strongly dependent on the solvent. The first step (Figure 15) is the proton transfer to form an ionpair intermediate, followed by addition of the carboxylic acid to form the O-acylisourea, a very reactive intermediate that is attacked by the amino group to form the amide. However, the Oacylisourea may rearrange to the unreactive $\mathrm{N}$-acylurea, or attack another carboxylic acid to form the symmetrical anhydride, which is also an excellent acylating agent.

Activated $\mathrm{N}$-acyl $\alpha$-amino acids and $\mathrm{N}$-alkoxycarbonyl $\alpha$-amino acids may cyclize to afford $5(4 H)$-oxazolones. N-Acyl derivatives are more prone to intramolecular cyclization than $\mathrm{N}$ alkoxycarbonyl derivatives because the oxygen carbonyl of amides is more nucleophilic than that of urethanes. 5(4H)-Oxazolones are also acylating agents albeit less powerful than the Oacylisourea or the symmetrical anhydrides. Tautomerization to the enol form results in racemization. Racemization is an important side reaction that may occur directly from the alphaamino acid or via the formation and enolization of the 5(4H)-oxazolone. Racemization and poor 
yields are due to the unreactive $\mathrm{N}$-acylurea. Solvents of low dielectric constants minimize both reactions (Scheme 30).

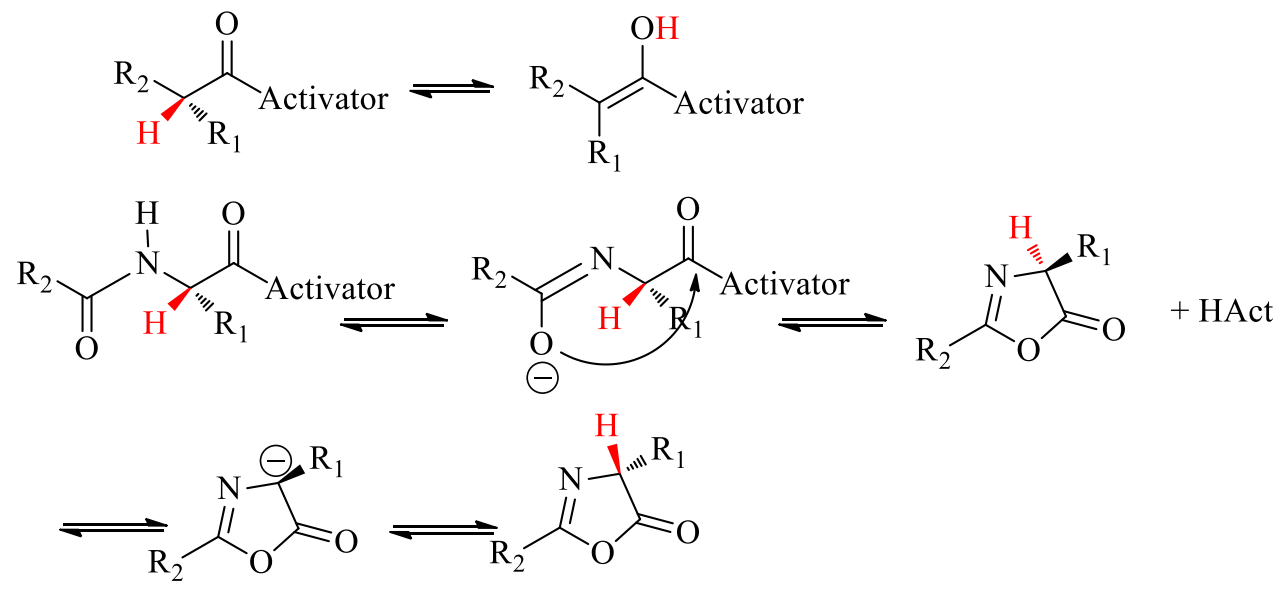

Scheme 30. Racemization under basic conditions.

The oxazolone reaction may be considered related to the Dakin-West reaction that involves the conversion of carboxylic acids to ketones. When amino acids are heated with symmetrical acid anhydrides in the presence of bases, $\alpha$-acyl amino ketones are formed and intramolecular acyl substitution affords oxazolones (Scheme 31).

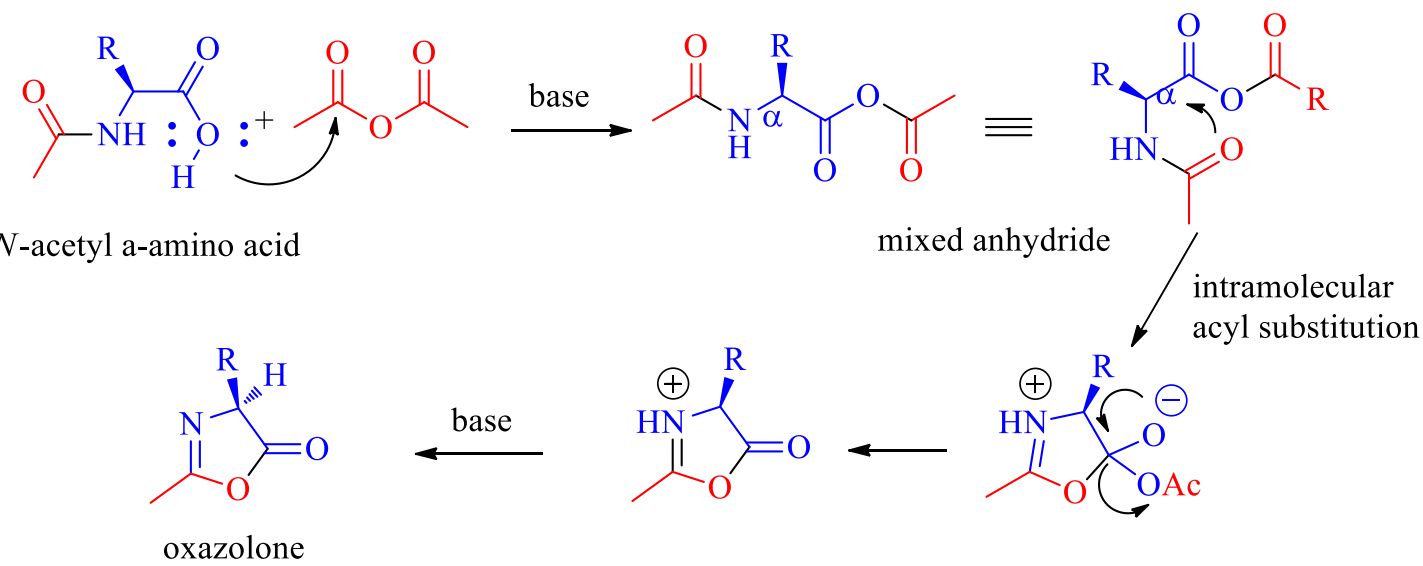

Scheme 31. Intermediate oxazolone in the Dakin-West reaction.

Oxazolones are activated towards aminolysis, and reaction with amino groups leads to amide bonds - but racemization via stabilized anions is fast compared to the rate of peptide bond formation. When the amino group of the activated residue is acylated with a simple acyl group or with a peptide chain, cyclization to the oxazolone occurs easily with most leaving groups. Oxazolone formation is not so easy when the acyl substituent is an alkoxycarbonyl protecting 
group. Alkoxyoxazolones (Figure 17) are less easily racemized, and undergo aminolysis more easily than oxazoles derived from simple acylamino acids. ${ }^{36}$

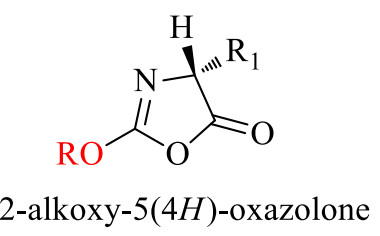

Figure 17. Alkoxyoxazolone structure.

Z-Boc-, and Fmoc- protected amino acids are less susceptible to racemization under normal conditions. The difference between these and simple acyl amino acids may be explained in terms of the lower $\mathrm{NH}$ acidity of the carbamates.

Racemization can be controlled by the rapid addition of a nucleophile to the $O$-acylisourea before side reactions occur. An acylating agent of lower potency, which reacts with amines but is more discriminating and does not undergo racemization, such as $\mathrm{N}$-hydroxysuccinimide or 1hydroxybenzotriazole, may also be used (Scheme 32).

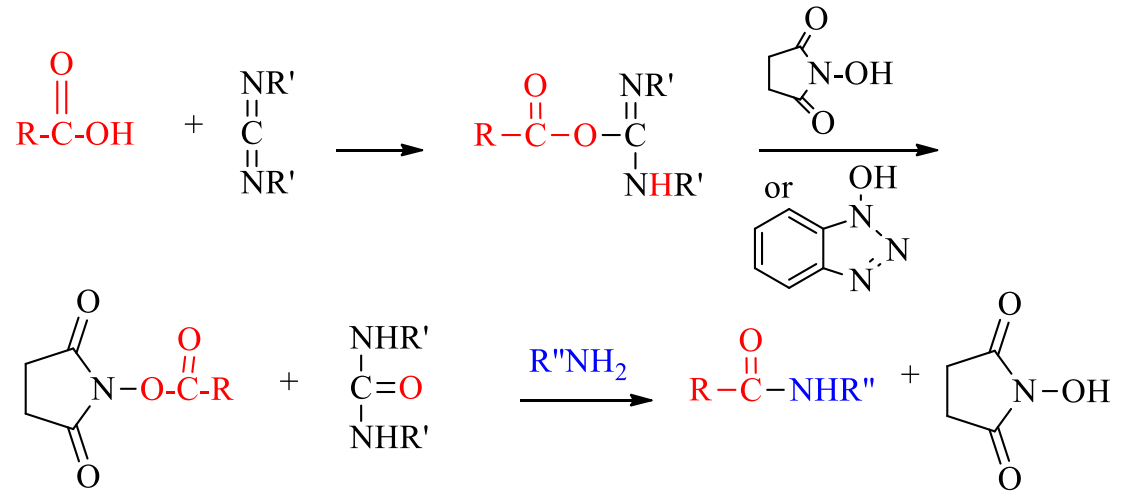

Conditions: $1: 1: 1: 1 \mathrm{RCO}_{2} \mathrm{H}: \mathrm{R}^{\prime \prime} \mathrm{NH}_{2}: \mathrm{DCC}: \mathrm{HOSu}$ or

$\mathrm{HOSu} / \mathrm{DMF} / 0{ }^{\circ} \mathrm{C}$ to $20{ }^{\circ} \mathrm{C}$

Scheme 32. Control of racemization by N-hydroxysuccinimide.

Formation of the ion-pair intermediate explains why the activation of the carboxylic acid is slower in the presence of base. Once the activated species is formed, base accelerates the coupling reaction. DCC activation is very dependent on solvents. It is rapid in solvents with low dielectric constants such as $\mathrm{CHCl}_{3}$ or $\mathrm{CH}_{2} \mathrm{Cl}_{2}$ where the formation of the $O$-acylisourea is instantaneous. Activation in dipolar aprotic solvents such as DMF, which may have to be used because of solubility problems, does not produce an immediate reaction and a complex mixture is formed. Activation is slower in a polar solvent. 
An equilibrium may be set up between the carboxylic acid and the $O$-acylisourea and, in the absence or presence of a second equivalent of acid, formation of the symmetrical anhydride occurs because reaction of the $O$-acylisourea with the unreacted carboxylic acid is faster than the addition of acid to the carbodiimide. The separate existence of acyclic $\mathrm{O}$-acylisoureas has not been confirmed. ${ }^{37}$

Although dicyclohexylurea is sparingly soluble, it may precipitate incompletely from the reaction mixture, and separation from the product may be difficult. Other carbodiimides are available, and they often have advantages over DCC — such as the water soluble $N$-ethyl- $N^{\prime}$ dimethylaminopropylcarbodiimide, (EDCI or EDC) $\mathrm{EtN}=\mathrm{C}=\mathrm{N}\left(\mathrm{CH}_{2}\right)_{3} \quad \mathrm{NMe}_{2} \bullet \mathrm{HCl}$. This carbodiimide is water soluble, and easily distillable, and it forms a more soluble urea, which can be separated from the protected peptide product by extraction in an acidic aqueous phase. The goal of many investigators is to find carbodiimides that afford ureas soluble in $\mathrm{CH}_{2} \mathrm{Cl}_{2}$, while retaining their original activity. $N, N^{\prime}$-diisopropylcarbodiimide, $\left(\mathrm{CH}_{3}\right)_{2} \mathrm{CH}-\mathrm{N}=\mathrm{C}=\mathrm{N}-\mathrm{CH}\left(\mathrm{CH}_{3}\right)_{2}$ (DIC), has been used in DMF, but it is not soluble in methylene chloride. This compound has been used in DMF and DMF/ $\mathrm{CH}_{2} \mathrm{Cl}_{2}$. N-Methyl-N'-tert-butylcarbodiimide, $\left(\mathrm{CH}_{3}\right)_{3} \mathrm{C}-\mathrm{N}=\mathrm{C}=\mathrm{N}$ $\mathrm{CH}_{3}$ and $N$-ethyl-N'-tert-butylcarbodiimide, $\left(\mathrm{CH}_{3}\right)_{3} \mathrm{C}-\mathrm{N}=\mathrm{C}=\mathrm{N}$-Et have also been suggested, but they are less effective and have a greater tendency to form $N$-acyl ureas.

Recently, $\quad N$-cyclohexyl-N'-tert-butylcarbodiimide, $\quad\left(\mathrm{CH}_{3}\right)_{3} \mathrm{C}-\mathrm{N}=\mathrm{C}=\mathrm{N}-\mathrm{C}_{6} \mathrm{H}_{11}$ has been proposed. Its urea is soluble in $\mathrm{CH}_{2} \mathrm{Cl}_{2}$. and its activity is comparable to, and even better than, DCC. A newer carbodiimide, 1,3-bis(2,2-dimethyl-1,3-dioxolan-4-ylmethyl)carbodiimide (BDDC) has been used for solution- phase peptide couplings with minimal epimerization (Figure $18)$.

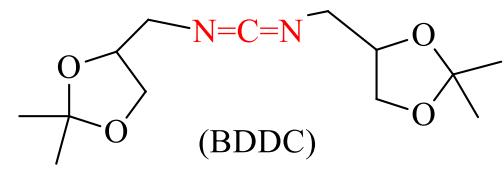

Figure 18. 1,3-Bis(2,2-dimethyl-1,3-dioxolan-4-ylmethyl)carbodiimide (BDDC).

During HODhbt/DCC-mediated peptide couplings, side products may be formed due to a competing ring-opening reaction. HODhbt $(2$ moles), and DCC ( $1 \mathrm{~mol})$ can react to form an $O$ azidobenzoyl active ester that reacts further with a free amino residue, leading to chain termination.

HODhat reacts similarly either with DCC or EDC.HCl. In both cases, the intermediate 3-(3'azidopicolinoyloxy)-4-oxo-3,4-dihydro-5-azabenzo-1,2,3-triazine was isolated (Figure 19). 
<smiles>Nc1cccnc1C(=O)On1nnc2ccccc2c1=O</smiles>

$o$-azidopicolinoyloxy active ester intermediate

Figure 19. 3-(3'-Azidopicolinoyloxy)-4-oxo-3,4-dihydro-5-azabenzo-1,2,3-triazine.

Coupling reactions may be carried out using the system diisopropylcarbodiimide/1-hydroxy7-azabenzotriazole. This system is of greater efficiency to preserve configuration during peptide segment coupling than the analogous DIC/HOBt system, in both dimethylformamide and dichloromethane.

The introduction of electron-withdrawing groups such as trifluoromethyl or nitro groups into the HOBt nucleus does not improve the reactivity.

The use of unsymmetrical alkyl arylcarbodiimides, or those bearing a tertiary amino substituent, whether protonated or quaternized, is less effective than that of neutral diisopropylcarbodiimide. In the case of stepwise peptide assembly, the efficiency of the system may be increased by carrying out the pre-activation step in the presence of 2,4,6-collidine $(2,4,6$ trimethylpyridine).

\subsection{Pyrocarbonates}

The stability and innocuous by-products of their reactions make pyrocarbonates superior to chloroformates (Figure 20). ${ }^{38}$

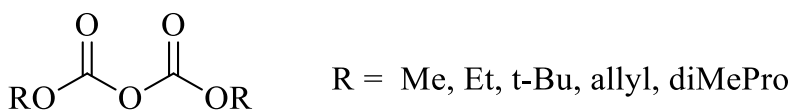

Figure 20. Pyrocarbonates.

Diethylpyrocarbonate (DEPC): Has been used for the derivatization of histidine under mild conditions (Scheme 33).

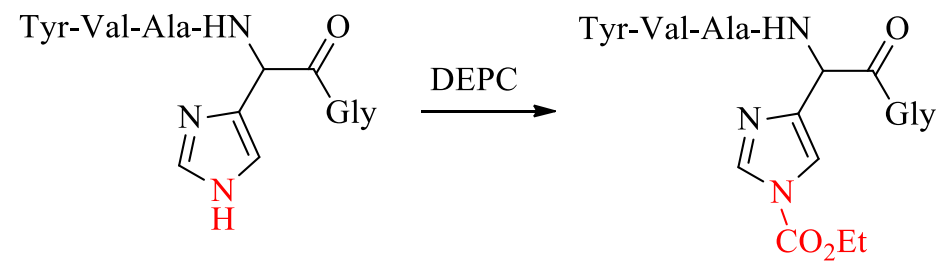

Scheme 33. Derivatization of histidine by DEPC. 
Di-t-Butyl dicarbonate (Boc anhydride) introduces Boc groups, which are easily removed under strong acid conditions. However, the $N$-Boc moiety functions not only as a protecting group, but it may react intra- and inter-molecularly with both nucleophiles and electrophiles. It has been used in efficient syntheses of various heterocycles. ${ }^{39}$

Diallyl pyrocarbonate introduces a common amino- protecting group, allyloxycarbonyl (Alloc), which can be removed selectively in the presence of other protecting groups (Scheme $34)$.

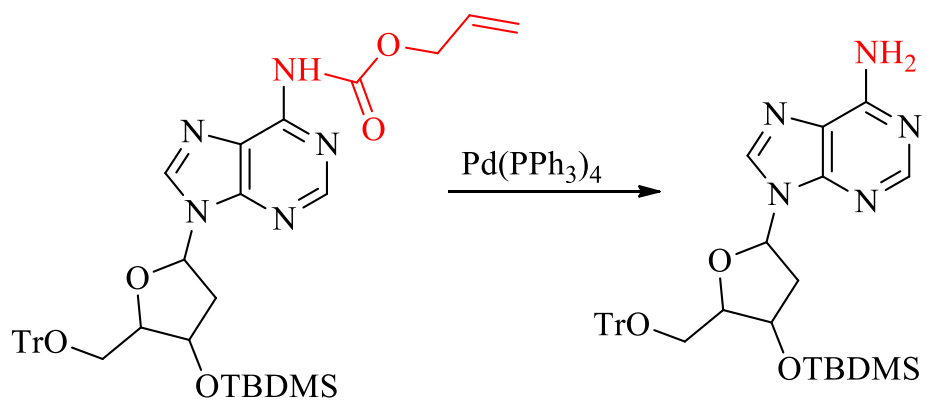

Scheme 34. Removal of the Alloc protecting group.

\subsection{Isoxazolium salts}

Isoxazolium salts have been known for many years, and used for carboxylic group modification in proteins. ${ }^{40}$ Isoxazolium salts are used as coupling reagents, usually in acetonitrile. Some wellknown isoxazolium salts are shown in Figure 21.

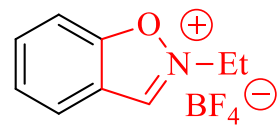

$N$-ethylbenzisoxazolium tetrafluoroborate

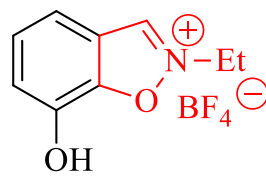

$N$-ethyl-7-hydroxybenzisoxazolium tetrafluoroborate

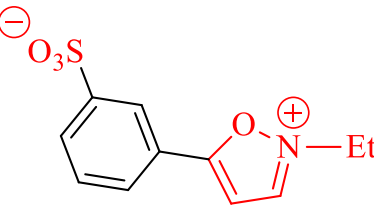

$N$-ethyl-5-phenylisoxazolium-3'-sulfonate (Woodward's reagent $\mathrm{K}$ )

Figure 21. Isoxazolium salts.

Woodward's reagent $K$ is commercially available. This reagent activates the carboxyl group of protected amino acids or peptides by producing an enol ester. This process gives high yields and, more importantly, the carboxyl groups of serine, threonine, and tyrosine can be activated without protection of the side- chain hydroxyls. Its disadvantages are the rearrangement of the enol ether to an imide, and the limited choice of solvents. The recommended solvents, acetonitrile or nitromethane, are not applicable to long polypeptide chains. It is also soluble in DMF, methylene chloride, and water. Appreciable racemization also occurs with the activation 
of acyl peptides.

Isoxazoles undergo ring opening with bases. The base-catalyzed degradation of isoxazolium salts is easy requiring only alkali metal carboxylates (Scheme 35).
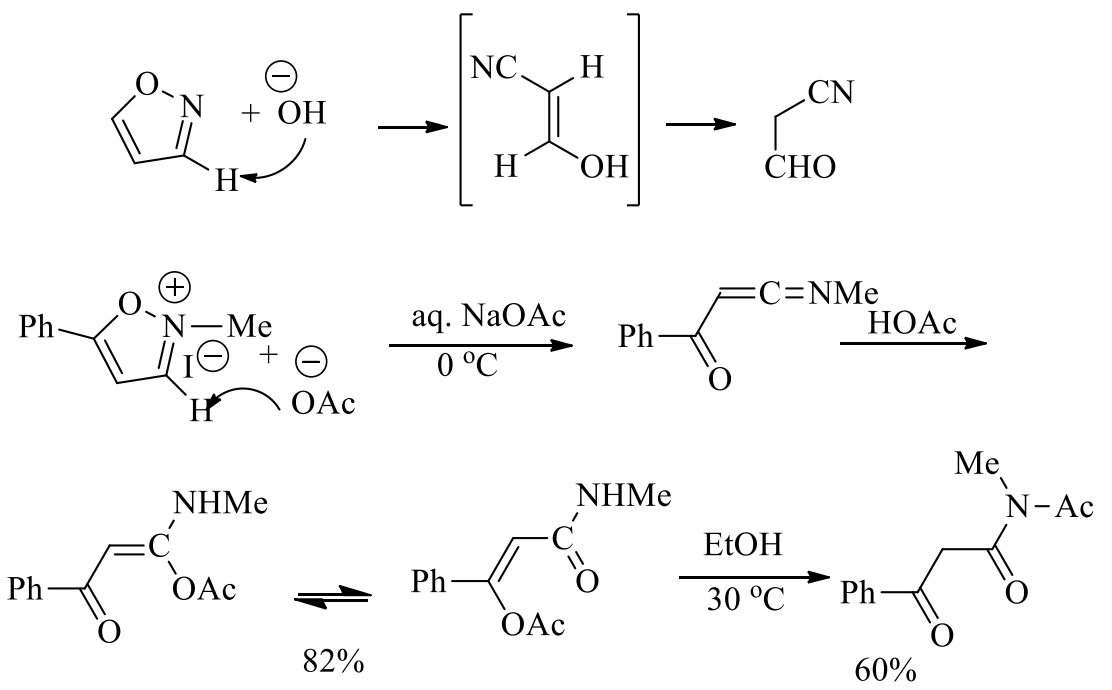

Scheme 35. Degradation of isoxazolium salts.

The coupling reaction is shown in Scheme 36. 


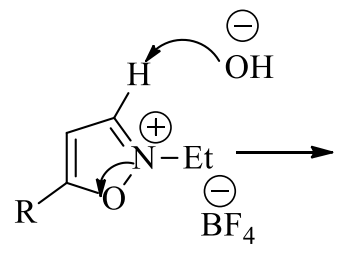<smiles>[R]C(=O)C(=C=NCC)NCC</smiles>

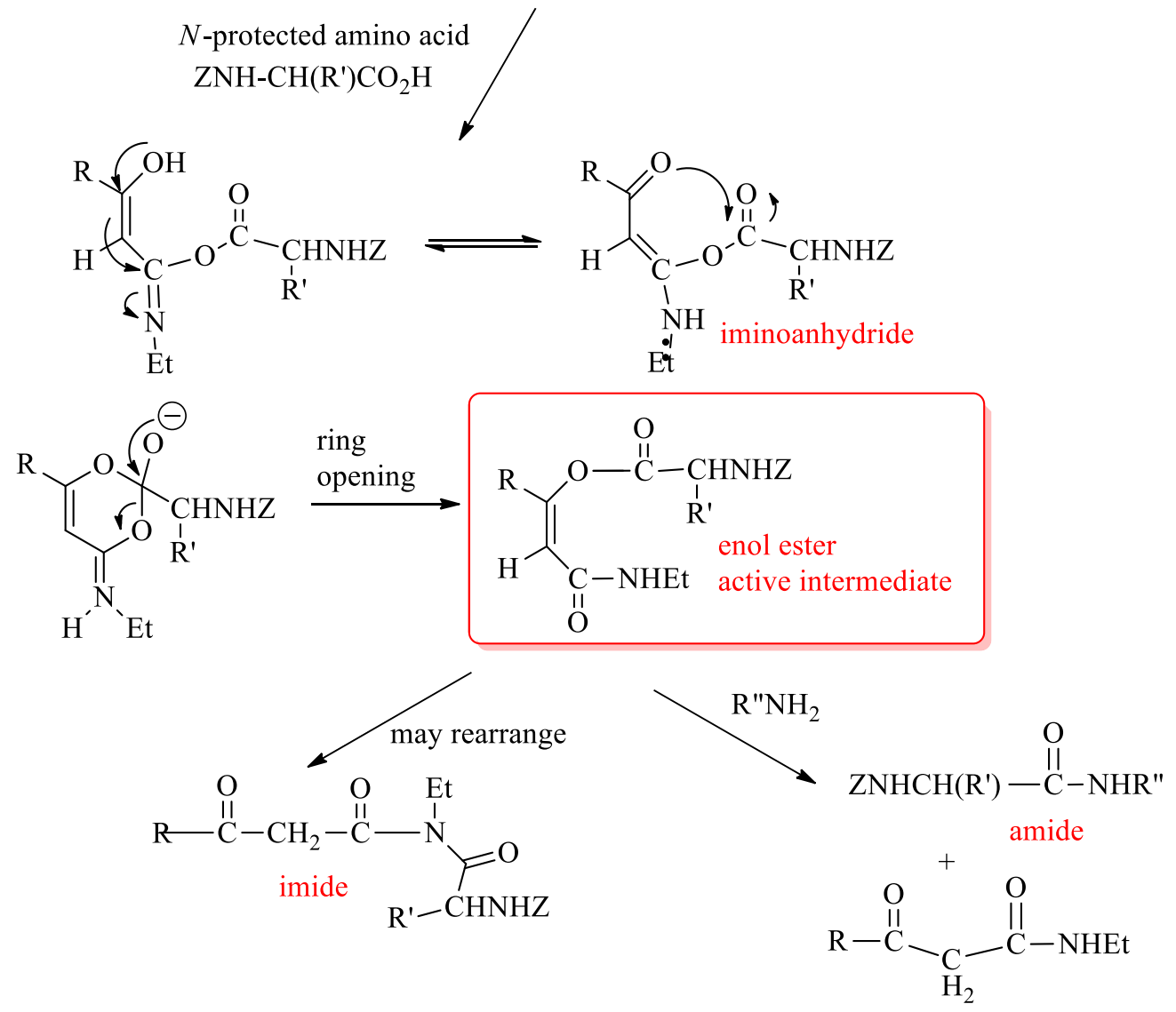

Scheme 36. Coupling reaction of isoxazolium salts.

\subsection{Phosphonium and phosphinic salts}

As noted previously, acyloxyphosphonium species are generated by the attack of carboxylate anions on phosphonium cations, and they react readily with nucleophiles. Since the early 1970s, ${ }^{41,42}$ large numbers of phosphonium salts have been developed for use as direct coupling reagents. They have the advantage of not yielding guanidinium by-products. The activation of carboxylic acids by phosphonium salts was pioneered by several investigators among them Kenner, Castro, Hruby, and Yamada. Treatment of hexamethylphosphorous triamide (HMPT) with various chlorides and anhydrides paved the way for the synthesis of phosphonium salts that were then used as acylating agents. Several phosphonium reagents and their abbreviations are shown in Figure 22. 


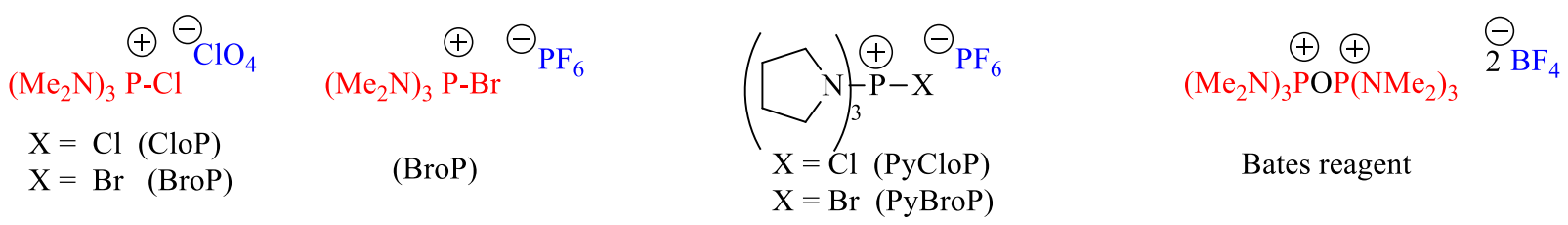<smiles>CN(C)[Po+]On1nnc2ccccc21</smiles>

(BOP)<smiles>CC(C)(C)P(On1nnc2ccccc21)P1CC2CCC1C2</smiles>

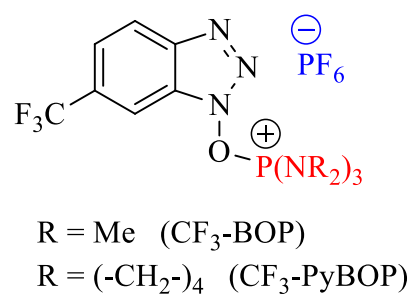<smiles>[R]C(=[R])CN([R16])On1nnc2ccc([N+](=O)[O-])cc21</smiles><smiles>[R16][PH2+]On1nnc2cccnc21</smiles>

$\mathrm{R}=\mathrm{Me}(\mathrm{AOP})$

$\mathrm{R}=\left(-\mathrm{CH}_{2}-\right)_{4} \quad($ PyAOP $)$<smiles>CC[Y](c1c(F)c(F)c(F)c(F)c1F)P(C)N1CC2CCC(C2)C1</smiles>

$\mathrm{X}=\mathrm{O} \quad$ (PyPOP)

$\mathrm{X}=\mathrm{S}$ (PyPSP)<smiles>CC(=O)N(C)CCN(C)P(=O)(N(C)C)N1CCC1</smiles><smiles>C[P+](C)(OSc1ccccn1)N1CC2CCC(C2)C1</smiles>

(PyTOP)<smiles>C[Pb](C)(On1nnc2ccccc2c1=O)N1CCCC1</smiles>
(PyDOP)

Figure 22. Phosphonium salts.

Benzotriazolyloxy-tris(dimethylamino)hexafluorophosphate (BOP) (Figure 23) is a stable crystalline solid. ${ }^{43}$ It led the way for the preparation of many other similar reagents.<smiles>CN(C)P(=N)(On1nnc2ccccc21)N(C)C</smiles>

Figure 23. Benzotriazolyloxy-tris(dimethylamino)hexafluorophosphate (BOP).

The carboxyl group must be in its anionic form, and for this reason a tertiary amine is added. An equivalent of the reagent is added to a 1:1 mixture of the amine and the carboxylic acid in a 
suitable inert solvent. Many pathways are possible but the main one is probably via the ester of HOBt (Scheme 37). Two possible modes of attack are possible, as shown in Scheme 38.

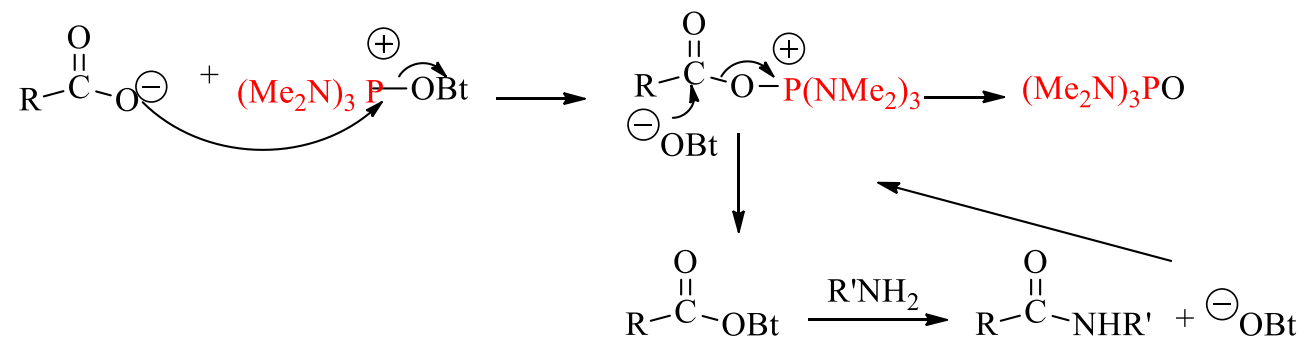

1:1:1:1 proportions of $\mathrm{RCO}_{2} \mathrm{H} / \mathrm{R}^{\prime} \mathrm{NH}_{2} / \mathrm{BOP} / \mathrm{DIPEA} /$ inert solvent $20^{\circ} \mathrm{C}$

Scheme 37. Activation by BOP.

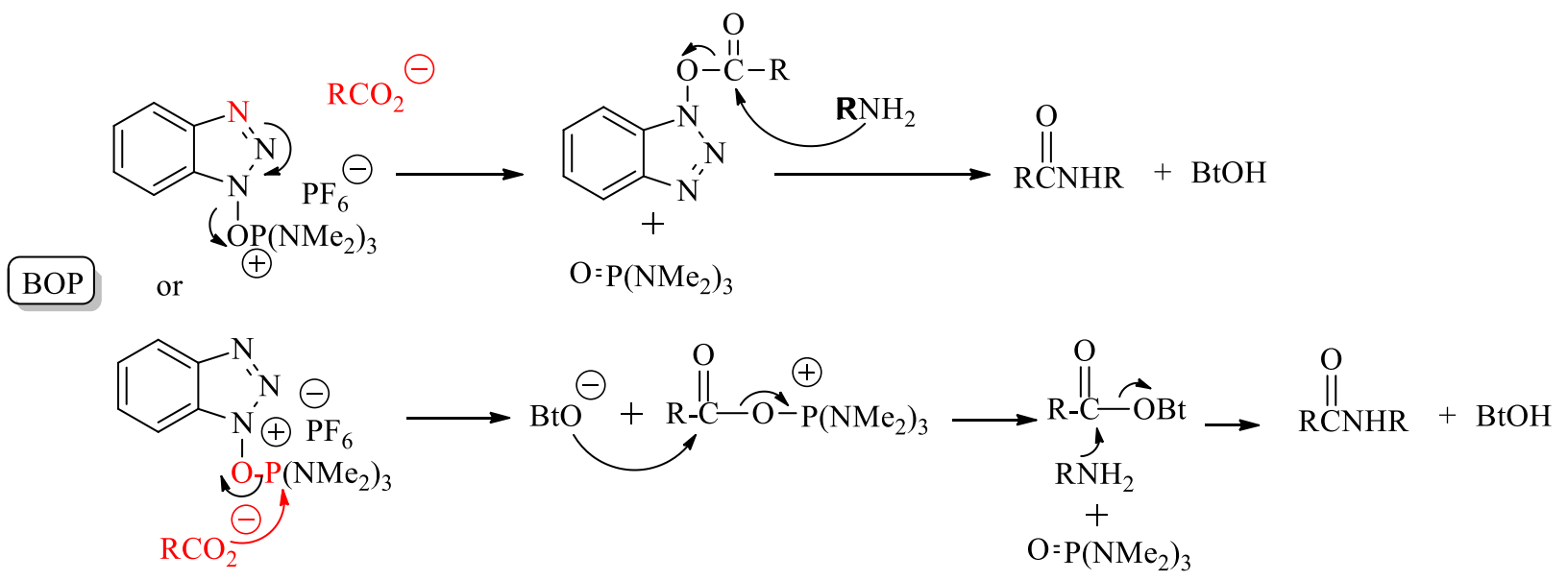

Scheme 38. Possible modes of attack on BOP.

All by-products are easily removed and the yields are usually good, with few side reactions. As the initial step is an anion-cation reaction, it is favored in non-polar solvents but any inert solvent may be used. There is, however, a safety concern with the use of BOP. The hexamethylphosphoric triamide (hexamethylphosphoramide), HMPA, by-product is a highly toxic substance. [One should not confuse HMPA with HMPT (hexamethylphosphorous triamide)] For this reason, other related salts that produce less toxic by-products have been developed. Some are more efficient than BOP in the formation of hindered peptides. Bromotris(dimethylamino)phosphonium hexafluorophosphate $(\mathrm{BrOP})$ exhibits superior reactivity in the coupling of $N$-methyl amino acids. It gives high yields, short reaction times, and no appreciable epimerization. Also, BrOP was found to be superior to BOP, bis- (2-oxo-3oxazolidinyl)phosphorodiamidic chloride (BOP-Cl) and diphenylphosphinic chloride (Dpp-Cl). 
The most reactive phosphonium salt, PyAOP, is expensive. The benzotriazole derivative PyBOP, is also more expensive than its analog, but it is especially useful for cyclization steps for the activation of hindered amino acid, ${ }^{44}$ while the use of aminium salts lead to the formation of guanidine derivatives. The PyBOP can be used in excess, and added during the coupling step. The derivatives may contain pyrrolidine as an impurity that can react with the carboxylic acid to give the corresponding pyrrolidine derivatives. ${ }^{45}$

\subsection{Immonium salts}

Immonium salts differ from uronium salts by the replacement of the central amino group with a hydrogen, alkyl or aryl group. Novel HOBt and HOAt- derived immonium type coupling reagents BDPMP, BPMP, and AOMP were shown to be highly reactive, and very efficient for peptide synthesis, affording low racemization and good yields. ${ }^{46} \mathrm{~N}$-(1H-Benzotriazol-1ylmethylene)- $N$-methylmethanaminium hexachloro antimonate (BOMI) was shown to exist as the $N$-substituted form rather than as the $O$-substituted form. The reagents are readily prepared from condensation of the corresponding amides with bis-(trichloromethyl)carbonate to yield immonium chlorides which are stabilized with antimonium pentachloride, and subsequently reacted with the potassium salt of 1-hydroxybenzotriazole or 1-hydroxy-7-azabenzotriazole in the presence of a tertiary amine as shown in Scheme 39.

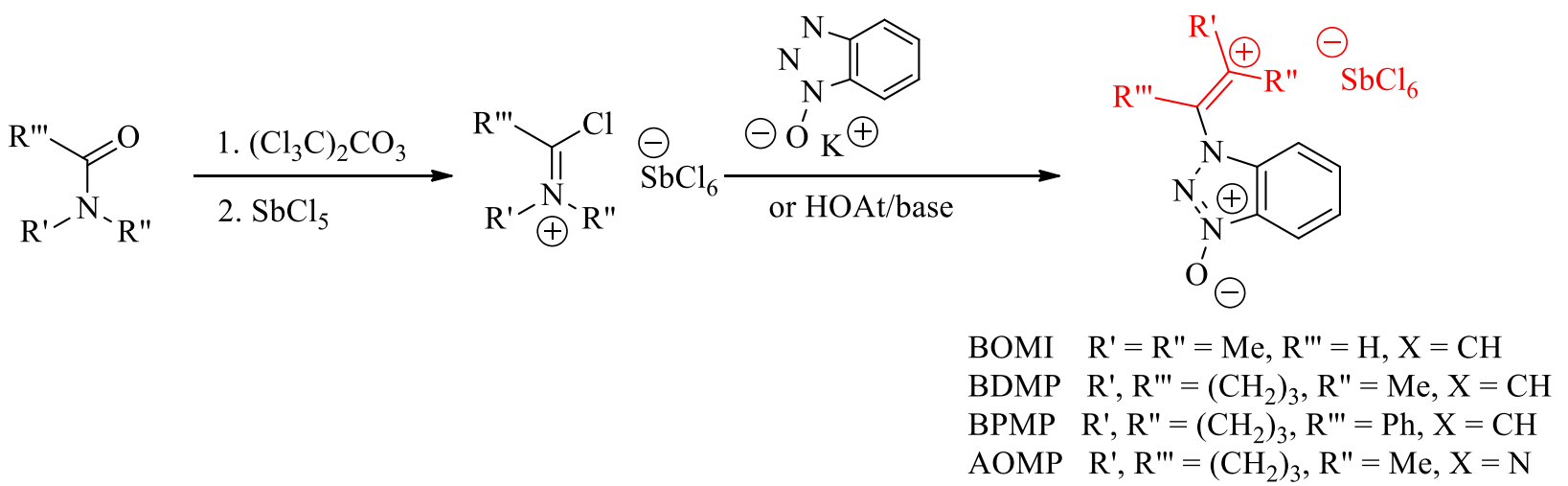

Scheme 39. Synthesis of immonium salts.

BDMP was used successfully in the synthesis of cyclosporin $\mathrm{O} \cdot{ }^{47}$ Immonium-type reagents exhibit higher reactivity and better racemization-suppressing ability than the corresponding uronium-or phosphonium-type reagents. BOMI, BDMP and BMP were more efficient than the uronium/aminium reagent HAPyU. BDMP was the best reagent in terms of high efficiency, low racemization and good yields. ${ }^{46}$

The higher reactivity of BDMP over that of a preformed active ester Z-G-F-OBt is probably due to the O-form isomer of the benzotriazolyl ester intermediate II, which is more reactive than the corresponding N-oxide isomer III (k4>k5) (Figure 32). Under mild reaction conditions, the initially generated active ester from the BDMP- mediated reaction exists as the O-acylated 
isomer II, and reacts rapidly with the amino component to afford product $(\mathrm{k} 4>\mathrm{k} 3)$, while in the case of the preformed Z-G-F-OBt, a certain amount of the less reactive $\mathrm{N}$-acylated isomer III is involved.

The proposed mechanism was confirmed by spectroscopic data. The O-isomer is characterized by absorption at $1805-1825 \mathrm{~cm}^{-1}$ in the infrared region. Z-G-F-OBt exists to some extent in the $\mathrm{N}$-acylated form, shown by the UV absorption at 328 and $342 \mathrm{~nm}$, and this isomer appeared to a greater extent in acetonitrile rather than in chloroform. The benzotriazolyl ester exists in two forms, the less polar $\mathrm{O}$-acylated isomer and the more polar $\mathrm{N}$-acylated isomer, and these can be separated. Either of the pure isolated isomers gradually equilibrates in solution into a mixture of the two forms (Scheme 40).

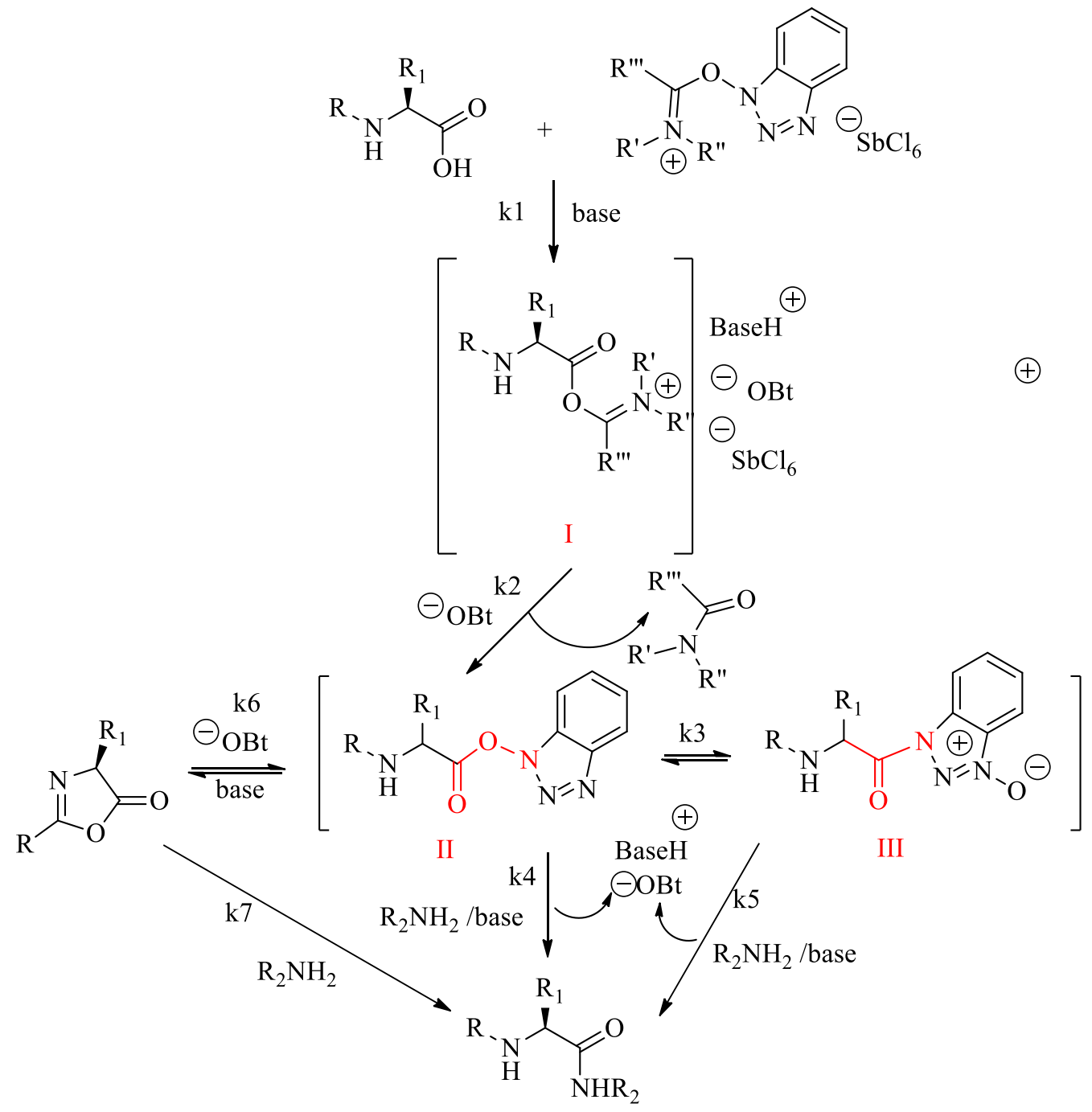

Scheme 40. Proposed mechanism for the HOBt-derived immonium salt coupling reaction. 


\subsection{Phosphonic acid derivatives}

A wide variety of phosphonic acid derivatives is available. Diphenylphosphoryl azide (diphenylphosphorazide, diphenylazidophosphonate) (DPPA) and diethylphosphoryl cyanide were the first phosphonic acid derivatives to be used in peptide synthesis. DPPAS reacts with free carboxyl groups to form acid azides. ${ }^{16}$ Other related phosphonic acid derivatives are shown (Figure 24).
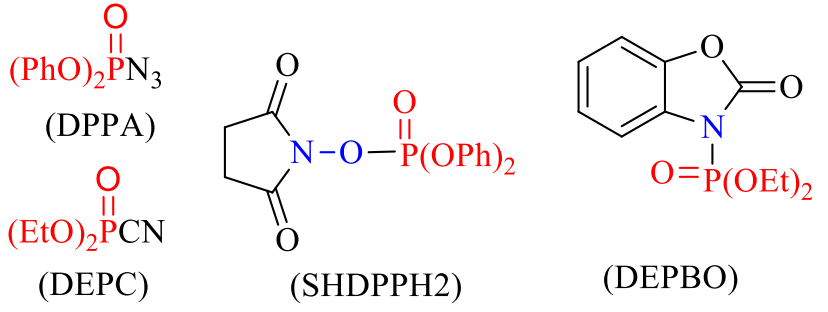<smiles>O=c1oc2ccccc2n1P1(=O)OCCCO1</smiles>

(DEPBO)

(DOPBO)<smiles>O=c1c2ccccc2nnn1OP1(=O)OCCO1</smiles>

(DOPBT)<smiles>CCOP(=O)(OCC)On1nnc2ccccc2c1=O</smiles>

(DEPBT)<smiles>CCOP(=O)(OCC)ON1C(=O)C2C3C=CC(O3)C2C1=O</smiles>

(ENDPP)

Figure 24. Coupling reagents derived from phosphonic acids.

These include $N$-diethoxyphosphorylbenzoxazolone (DEPBO), and $N$-(2-oxo-1,3,2dioxaphosphorinanyl)benzoxazolone (DOPBO) that have N-P links. Related organophosphorus compounds that have a N-O-P link are 3-[O-(2-oxo-1,3,2-dioxaphosphorinanyl)oxy]-1,2,3benzotriazin-4-(3H)-one (DOPTB), $N$-succinimidyl diphenylphosphate (SDPP), ${ }^{48} \quad 3-$ (diethoxyphosphoryloxy)-1,2,3-benzotriazin-4-(3H)-one (DEPBT) (Figure 25). ${ }^{49-51}$ These reagents are similar to benzotriazole derivatives such as benzotriazol-1-yl diethyl (ENDPP) and its diphenyl analog.

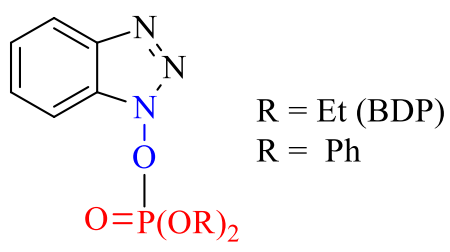

Figure 25. 3-(Diethoxyphosphoryloxy)-1,2,3-benzotriazin-4-(3H)-one (DEPBT).

The new crystalline phosphonate reagent DEPBT mediates amide bond formation with a remarkable resistance to racemization. ${ }^{51}$ A tertiary amine is required to generate the carboxylate of the N-protected amino acid. The transient intermediate rearranges with loss of diethyl 
phosphite to form the activated ester, which is then attacked by the amine to form the amide product (Scheme 41).
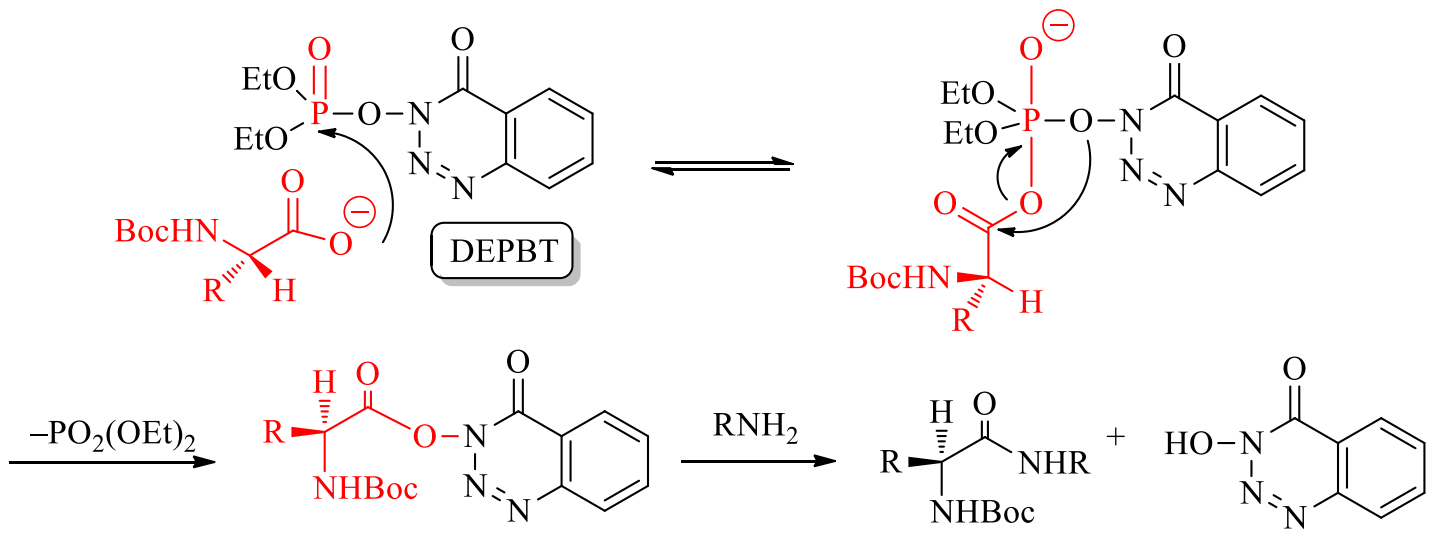

Scheme 41. Coupling mechanism of DEPBT reagent.

Pentafluorophenyl diphenylphosphinate (FDPP) is an efficient coupling reagent for the racemization-free synthesis of peptides (Figure 26). Before this reagent, the use of diphenylphosphonic mixed anhydrides, and pentafluorophenyl esters in peptide coupling was known. However, the need to pre-activate amino acids or to prepare active esters was unattractive. FDPP is used directly as a coupling reagent without pre-activation, the coupling procedure is easy, there is no insoluble product, the reaction yield is high, and the coupling is fast.

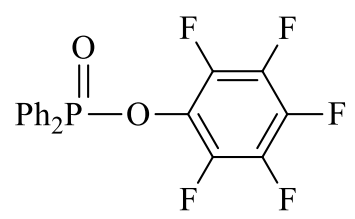

(FDPP)

Figure 26. Pentafluorophenyl diphenylphosphinate (FDPP).

A phosphorodiamidic chloride, bis-(2-oxo 3-oxazolidinyl)phosphorodiamidic chloride (BOP$\mathrm{Cl}$ ) is another peptide coupling reagent that is widely used especially for coupling of $\mathrm{N}$ methylamino acids. ${ }^{52}$ Its effectiveness is attributed to intramolecular base-catalysis by the oxazolidinone carbonyl of the mixed anhydride active species (Figure 27). 


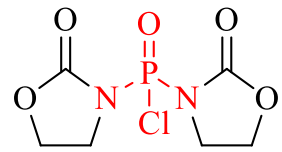

(BOP-Cl)

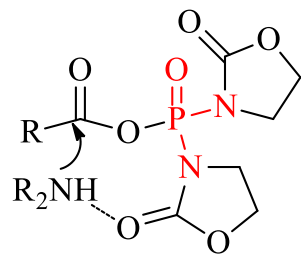

activated species

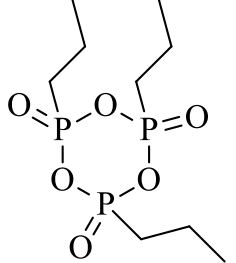

(PPAA)

Figure 27. Activation by bis-(2-oxo 3-oxazolidinyl)phosphorodiamidic chloride (BOP-Cl), and the structure of PPAA.

2-Propanephosphonic acid anhydride (PPAA) is a commercially available trimeric reagent that has been used in solution- phase peptide synthesis and cyclization reactions (Figure 27). It gave better results than BOP-Cl, and was found superior to HATU and HAPyU for cyclization reactions, especially for sterically hindered peptides. ${ }^{53-55}$

\subsection{Aminium salts}

Peptide coupling via uronium or phosphonium salts first proceeds by reaction of the $N$-protected carboxylic acid with the coupling reagent to form an active ester, which then reacts with the amino component to give the amide bond. The latter step is rate-limiting, and responsible for loss of configuration. The reactivity of the active ester intermediate is a critical factor in the evaluation of an individual uronium or phosphonium salt.

Couplings with aminium/uronium salts in the presence of base have proved to be more effective than those carried out with phosphonium reagents or carbodiimides in the presence of hydroxylamine derivatives. ${ }^{56}$ However, reagents based on phosphonium salts may be preferred to their aminium/uronium counterparts for cyclization reactions because the latter compounds can give guanilidation reactions on the amino group.

Aminium salts were initially assigned an uronium type structure in analogy with the related phosphonium salts. X-Ray structure determinations of HBTU and HATU have been reported. They have a positive carbon instead of phosphorus, and are actually guanidinium derivatives. They crystallize as aminium salts, as the guanidinium $\mathrm{N}$-oxide isomers. ${ }^{57}$ In solution the two forms could co-exist, since either could react with a carboxyl group to give the activated ester intermediate. $N$-[(dimethylamino)-1H-1,2,3-triazole[4,5-b]pyridin-1ylmethylene]$N$-methylmethanaminium hexafluorophosphate (HATU) and O-(benzotriazol-1-yl)-1,13,3tetramethyluronium hexafluorophosphate (TBTU) (Figure 28). 


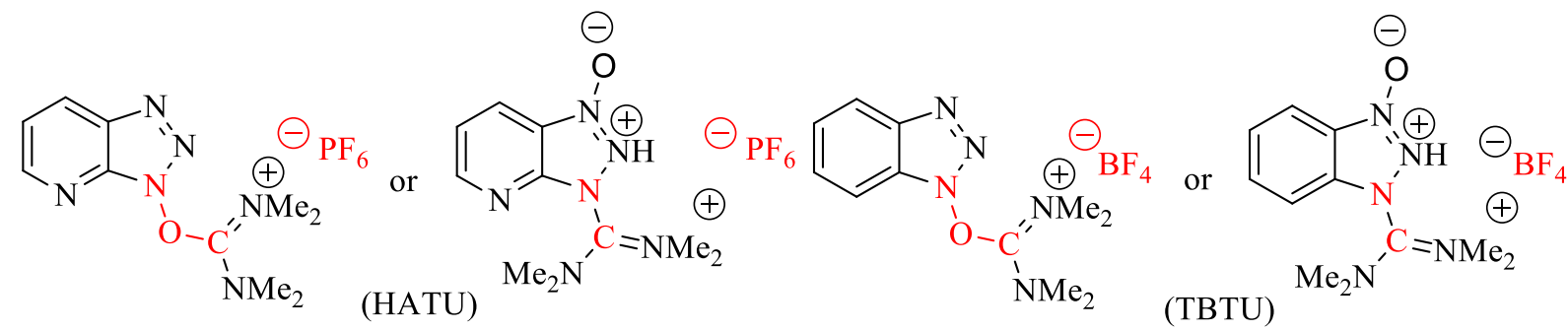

Figure 28. Aminium salts.

These reagents are useful in hindered couplings and give minimum racemization. HATU appears to involve the formation of 7-azabenzotriazol-1-yl esters that are highly active probably because of intramolecular general base-catalysis (Figure 29).

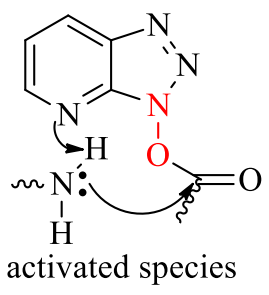

Figure 29. Activation by HATU.

Phosphonium salts are less stable than the corresponding aminium/uronium derivatives in the absence of base. In the presence of a base, phosphonium salts are somewhat more stable, suggesting that aminium/uronium salts are somewhat more reactive. ${ }^{58}$

The other reagents, O-(benzotriazol-1-yl)-1,1,3,3-tetramethyleneuronium hexafluorophosphate (HApyU) and O-(benzotriazol-1-yl)-1,13,3-pentamethyluronium hexafluorophosphate (TAPipU) are also excellent reagents. Using HAPyU and TAPipU in only $10 \%$ excess, a quantitative ring closure of decapeptide occurred within $30 \mathrm{~min}$, at high concentration $(0.1 M)$. Similar results were obtained with 3,4,5, and 7-membered residues. A 6membered ring- closure with all- L- residues took $30 \mathrm{~min}$ and gave a $55 \%$ yield $(<0.5 \%$ racemization).

The reactivity of various salts (aminium/uronium and phosphonium) derived from HOBt and HOAt was correlated to their structures. As seen earlier, the aza derivatives are more reactive than the parent benzotriazole derivatives in both activation and coupling. The structure of the carbon skeleton is an important element in determining the efficiency of the reagent for the activation step. The pyrrolidino derivatives (HAPyU) are superior to the piperidino derivatives (HAPipU) and those derived from trialkyl derivatives (HATU). The dihydroimidazole derivatives HAMDU and HBMDU, as well as HAMTU, are inefficient because they are unstable in DMF in the presence of base (DIEA), so they decompose before activation of the carboxylic acid. Calculations were carried out on simplified systems to explain the behavior of the activated 
species. The reaction examined was the addition of water to these carbocation models and the examination of the corresponding transition states. The results revealed that the lone pairs associated with each nitrogen atom were delocalized within the atoms N-C-N, giving an allyl radical- anion character to the molecules. The differences found in the energy barriers for the transition states were ascribed to the pyramidalization or planarity of the nitrogens. The reactivity of the aminium/uronium salts was found to depend on two opposing effects: the pielectron delocalization between the N-C-N atoms and the strain within the carbon skeleton. Increased $p i$-electron delocalization disfavors nucleophilic attack. If the structure of the carbon skeleton reduces this electronic delocalization, nucleophilic attack becomes more favorable. ${ }^{56}$

The structures of the reagents used in the study are shown in Figure 30.

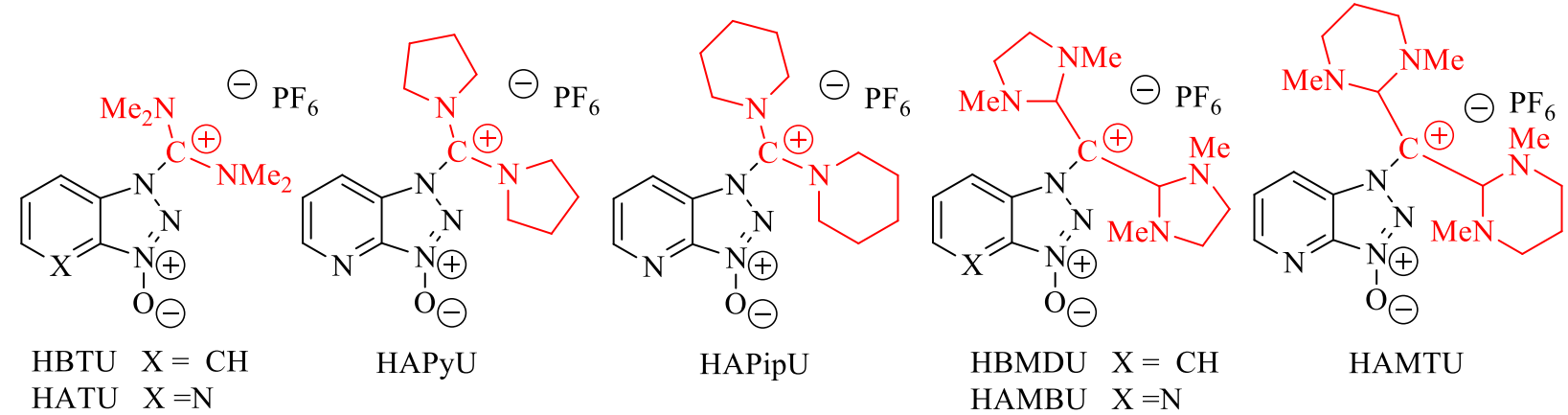

Figure 30. Aminium salts used in reactivity studies.

The most reactive aminium salt, HATU, is expensive and an alternative is to use HBTU/TBTU in the presence of $\mathrm{Cl}-\mathrm{OBt}$ that makes these reagents more reactive. The tetrafluoroborate counterion forms more soluble salts than the hexafluorophosphate, and allows for the preparation of more concentrated solutions. The racemization of HBTU and TBTU was investigated. TPTU/HOBt couplings were obtained virtually free of racemization and are the reagents of choice for segment condensations. TBDTU showed the lowest racemization in sensitive couplings, but it is subject to side reactions. Salts derived from dimethylamine are the most stable, and the pyrrolidino derivatives are of intermediate stability.

$O$-(3,4-Dihydro-4-oxo-1,2,3-benzotriazin-3-yl)-1,1,3,3-tetramethyluronium hexafluorophosphate (HTDU) is an excellent activating agent but the resulting active ester is less reactive that the corresponding HOAt and HOBt esters (Figure 31). ${ }^{59,60}$

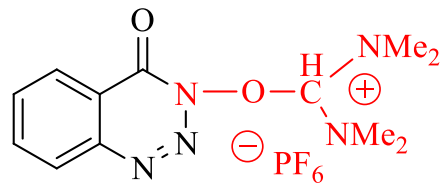

HDTU

Figure 31. Traditional structure of HTDU. 
All coupling reagents were more stable when the DMF solutions were kept under nitrogen. HBTU and TDBTU may decompose violently if dried at elevated temperatures. ${ }^{60}$

Correlation of the reactivity of different 'onium salts showed that aza- derivatives are more reactive than the parent benzotriazole derivatives in both activation and coupling, and that the activation step was determined by the structure of the carbon skeleton. Pyrrolidino derivatives were found superior to the piperidino and trialkylamino analogs.

Aminium salts can react with amino groups to form a guanidino derivative, a process that terminates peptide chain elongation. During the much slower activation of hindered amino acids, protected peptide segments, or carboxylic acids involved in cyclizations, the aminium salt may undergo reaction with the amino component. Phosphonium salts are not involved in such reactions and therefore are better reagents for activation of hindered species.

\section{Other Coupling Reagents}

\subsection{Organophosphorus esters}

The organophosphorus esters dimethoxyphosphinyloxy-7-azabenzotriazole (DepOAt), diphenoxyphosphinyloxy-7-azabenzotriazole (DpopOAt), 1-[di(o-tolyl)-phosphinyloxy]benzotriazole (DtpOBt), and 2,8-dimethylphenoxaphosphinyloxy-7-aza-benzotriazole (DmppOAt) were found to be excellent coupling reagents for forming peptide bonds, and to be generally superior to the uronium/guanidinium analogs and HOBt and HODhbt-derived phosphate ester counterparts in minimizing loss of configuration during segment coupling (Figure 32). The phosphinyl analogs are more shelf-stable than the phosphoryl derivatives. ${ }^{61}$

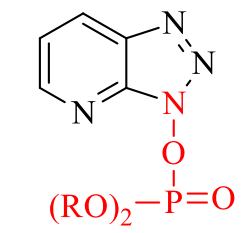

$\mathrm{R}=\mathrm{Et} \quad$ DepOAt $\mathrm{R}=\mathrm{C}_{6} \mathrm{H}_{5} \quad$ DpopOAt

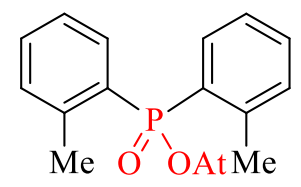

DtpOAt

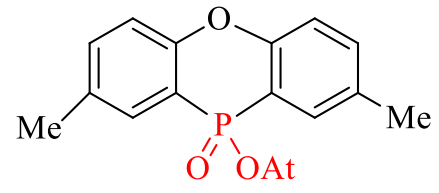

DmppOAt

Figure 32. Organophosphorous esters.

The phosphinyl esters were generally as efficient as the phosphate analogs. Their extended shelf- life stability makes them practical for general use. The speed of formation of the active ester is an important factor in evaluating the efficiency of a coupling reagent. The reagents were tested on the sterically hindered carbonyl group of $N$-benzyloxycarbonyl- $\alpha$-amino-isobutyric acid (Z-Aib-OH) and the corresponding active ester in both $\mathrm{DMF}$ and $\mathrm{CDCl}_{3}$. In DMF all tested reagents except for DtpOBt and DepODhbt, led to rapid formation of active esters. In the less polar solvent $\mathrm{CDCl}_{3}$, differences in reactivity were noted especially for DtpOBt and HBTU. The 
rapid activation observed for HDTU is not matched by its coupling activity, presumably due to the low reactivity of the active ester. Coupling reactions are completed more rapidly for the OAt systems than for the ODhbt or OBt systems because of the ease of formation and high reactivity of the OAt ester. The new phosphorus-based OAt derivatives are more effective in preserving configuration than other tested reagents including HATU. HAPyU sometimes achieved the same results as those obtained for the new phosphorus esters although DepOAt and DpopOAt gave lower epimerization levels.

Esters of sulfonic acids and HOBt related to those described for the phosphorus series have been used for peptide coupling. The methodology has not been applied widely because it depends on the basicity or reactivity of the amino component of the coupling process. The sulfonate esters for in situ coupling are often accompanied by formation of a sulfonamide by product. It is not known whether sulfonate esters are involved in an equilibrium similar to the $O$ acyl/ $\mathrm{N}$-acyl type. The neighboring- group effect observed for the OAt derivatives did not appear as important for the sulfonyl esters of HOAt and HOBt, as there was not a clear difference in their reactivity. Of the various sulfonate esters of HOAt that were examined, only the nitrosubstituted benzenesulfonyl esters reduced epimerization levels as observed for HATU. ${ }^{62}$

\subsection{Activated phosphate}

Utilization of a phosphate derivative as a component of a mixed anhydride is an accepted approach to an efficient coupling reagent when appropriate leaving groups such as chloro-, cyano-, heterocyclic-, and phosphate- groups are appended. A more powerful but less nucleophilic leaving group should provide an even more efficient coupling reagent. Trifluoromethanesulfonanilide was chosen for this purpose as this group was already known as a selective and rapid alkoxycarbonylation reagent (Figure 33). ${ }^{63}$ The reagent performed well in the coupling of bulky amines and carboxylic acids, in the presence of diisopropylethylamine and HOAt, in DMF. Coupling of various amines in methylene chloride and in the presence of proton sponge gave excellent yields of amides. A peptide coupling reaction in the presence of proton sponge and in DMF at room temperature, gave an $85 \%$ yield of product and with low racemization $(2 \%) .{ }^{64}$

$$
\mathrm{Ph}-\mathrm{N}_{\mathrm{PO}(\mathrm{OEt})_{2}}^{\mathrm{SO}_{2} \mathrm{CF}_{3}}
$$

activated diethyl phosphate

Figure 33. Structure of activated phosphate.

\subsection{Triazoles}

Arenesulfonyl-1,2,4-triazoles have also been used as coupling reagents. 1-(Mesitylenesulfonyl)3-nitro-1,2,4-triazole (TPNST) and 1-(2,4,6-tri-isopropylbenzenesulfonyl)-3-nitro-1,2,4-triazole (TPSNT) are examples of such reagents, as seen in Figure 34. It was reported that 
mesitylenesulfonic-carboxylate mixed anhydrides, symmetrical anhydrides, and carbonyl azolides are the active intermediates in the coupling reaction.

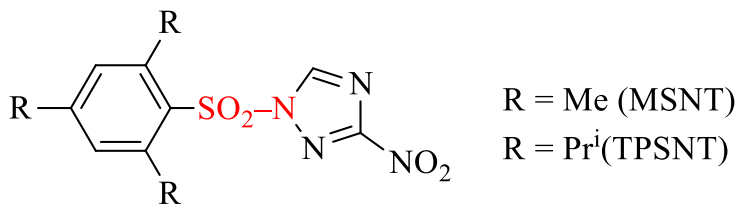

Figure 34. Coupling reagents derived from triazoles.

A mixture of 2-trifluoroacetylthiopyridine-1-hydroxybenzotriazole, the sodium salt of 1hydroxybenzotriazole, and an acylamino acid, was found to acylate amino acid esters in high yields with minimal racemization. ${ }^{65}$ The mixture is a highly effective coupling reagent. Addition of the acylamino acid to the mixture, and an activation period of a few minutes between $-20{ }^{\circ} \mathrm{C}$ and room temperature, is followed by allowing the mixture to warm for about 10 hours at room temperature. The yield of peptide is usually $80-85 \%$ and racemization between 0 and $1 \%$

\subsection{Triazines}

1,3,5-Triazines have also been used as coupling reagents. They are shown in Figure 35.<smiles>COc1nc(Cl)nc(OC)n1</smiles>

(CDMT)<smiles>CCCCOc1nc(OCCCCCC(F)(F)F)nc(OC(F)(F)C(F)(F)F)n1</smiles>

(TPfT)<smiles>COc1nc(OC)nc([N+]2(C)CCOCC2)n1</smiles>

(DMTMM)

Figure 35. Coupling reagents derived from triazines.

2-Chloro-4,6-dimethoxy-1,3,5-triazine (CDMT) is a commercially available crystalline compound. 2,4,6-tris-(Pentafluorophenoxy)-1,3,5-triazine (TPfT) may be prepared from cyanuric chloride and potassium pentafluorophenolate. Activation of carboxylic groups by means of 2chloro-4,6-disubstituted-1,3,5-triazines was found to be a multi-step process, with participation of quaternary triazinylammonium salts as intermediates, and with the rate depending strongly on the structure of the tertiary amine. The stereo-differentiating effects of amines prompted investigation of the activation stages of the carboxylic function in the process involving the substitution of chlorine in the triazine ring with the carboxylate anion. The mechanism involves two subsequent substitution reactions in the triazine ring. Substitution of the chlorine by a tertiary amine, leading to a quaternary ammonium salt, is sensitive to steric hindrance by the amine substituents. The second reaction involves substitution of the quaternary ammonium group 
by the carboxylate ion, affording the triazine activated ester. An addition-elimination mechanism was suggested for the addition of the amine to the chloro derivative.

The related triazine, 4-(4,6-dimethoxy-1,3,5-triazin-2-yl)-4-methylmorpholinium chloride (DMTMM), is an alcohol- and water- soluble condensing agent. It is a colorless air- and waterstable solid. ${ }^{66,67}$ It is a very efficient agent for condensing a variety of carboxylic acids and amides: both primary and secondary amines may be used. Because the reaction of carboxylate anions is faster, pre-mixing the carboxylic acid and the amine forms an ammonium carboxylate salt. The carboxylate anion reacts with the triazine to generate the active species (an acyloxytriazine) and $\mathrm{N}$-methylmorpholine. This prevents the direct addition of the amine to the reagent. The amine is regenerated from the ammonium cation by $N$-methylmorpholine that is liberated in the activation step. The amine then reacts with the activated ester to form the amide and the triazinone (Scheme 42).

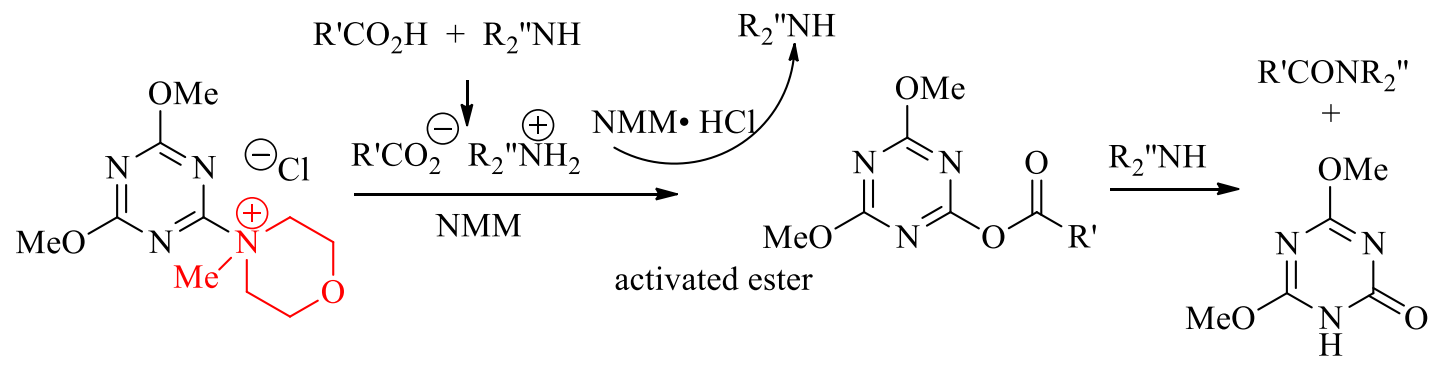

Scheme 42. Coupling mechanism of DMTMM.

The by-products are water-soluble and easily removed during the workup. No additives are required. DMTMM is susceptible to demethylation at the morpholinium nitrogen, to give chloromethane and 4-(4,6-dimethoxy-1,3,5-triazin-2-yl)morpholine (DMTM) when it is suspended in chloroform or dichloromethane. A small amount of DMTM is formed in tetrahydrofuran but is negligible in methanol. When DMTMM is dissolved in polar protic solvents such as methanol or water, the ions are well solvated; therefore the possible attack of the solvated chloride on the methyl group at the solvated morpholinium nitrogen should be very slow ,and unlikely to occur.

\subsection{Thiazolium Salts}

2-Bromo-3-ethyl-4-methylthiazolinium tetrafluoroborate (BEMT), a crystalline solid, is reported to be an efficient coupling reagent for hindered amino acids. It was used for the coupling of $N$ alkyl or $\alpha, \alpha$-dialkyl amino acids (Scheme 43). ${ }^{68}$ 


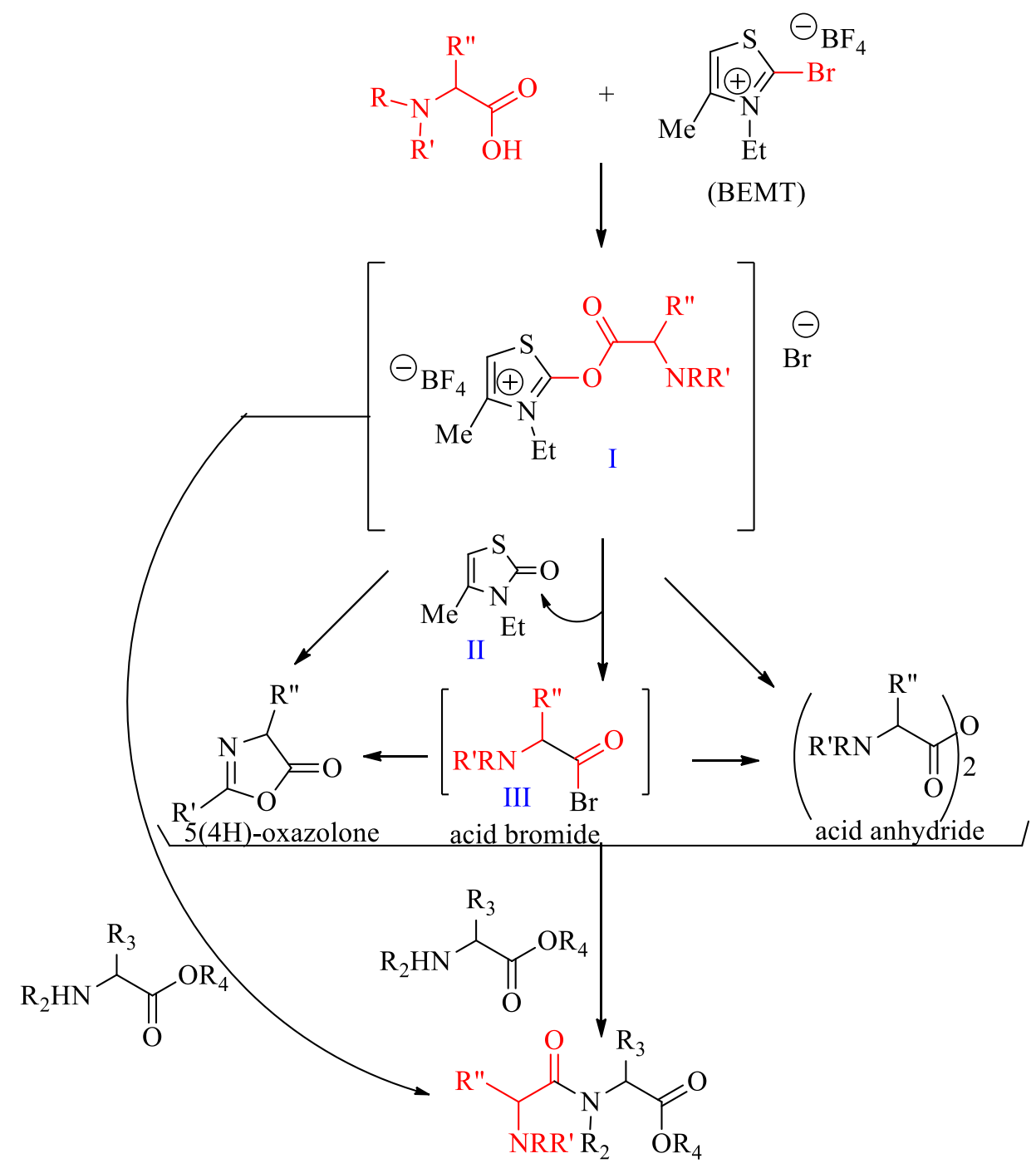

Scheme 43. Activation and coupling with BEMT.

A probable coupling mechanism involves the formation of an unstable acyloxythiazolium salt I that reacts with the amino- component directly, or is competitively converted into the acid bromide III, which is subsequently converted into the dipeptide by aminolysis. Small amounts of the corresponding acid anhydride and 5(4H)-oxazolone are also formed from the acyloxythiazolium salt or acid bromide. The main by-product of the reaction is $N$-ethyl-4-methyl thiazolidone II, which was isolated and characterized from the reaction mixture.

\subsection{Imidazolium and benzimidazolium salts}

$\alpha, \alpha$-Dimethyl amino acids are especially difficult to couple. A new coupling reagent was developed, 2-chloro-1,3-dimethylimidazolium hexafluorophosphate (CIP). This coupling reagent was compared to PyBOP. Additives for the CIP coupling were also examined (Figure 36). The 
order of enhancement was 1-hydroxy-7-azabenzotriazole 3-hydroxy-3,4-dihydro-4-oxo-1,2,3benzotriazine $>$ DMAP $>$ HOBt $(\mathrm{HODhbt}){ }^{69-71}$

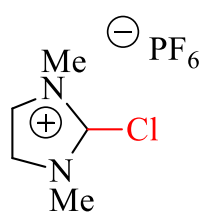

CIP<smiles>On1nnc2cccnc21</smiles>

HOAt<smiles>CN(C)c1ccncc1</smiles>

DMAP

Figure 36. 2-Chloro-1,3-dimethylimidazolium hexafluorophosphate (CIP) and additives.

A related reagent, 1,1'-carbonyl-bis-(3-methylimidazolium) triflate (CBMIT) (Figure 37), which is readily prepared by bis- alkylation of carbonyldiimidazole with methyl triflate, was shown to react with acids to form highly reactive acyl imidazolium intermediates that couple with amines and alcohols. ${ }^{72}$ The reagent may be used in the preparation of complex enantiomerically pure esters and peptides in high yields under remarkably mild conditions. The activation and coupling with CBMIT is shown in Scheme 44.

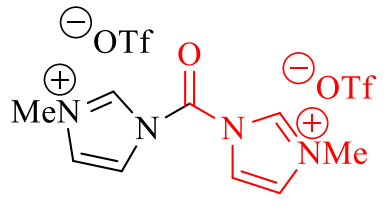

CBMIT

Figure 37. 1,1'-Carbonylbis(3-methylimidazolium) triflate (CBMIT).

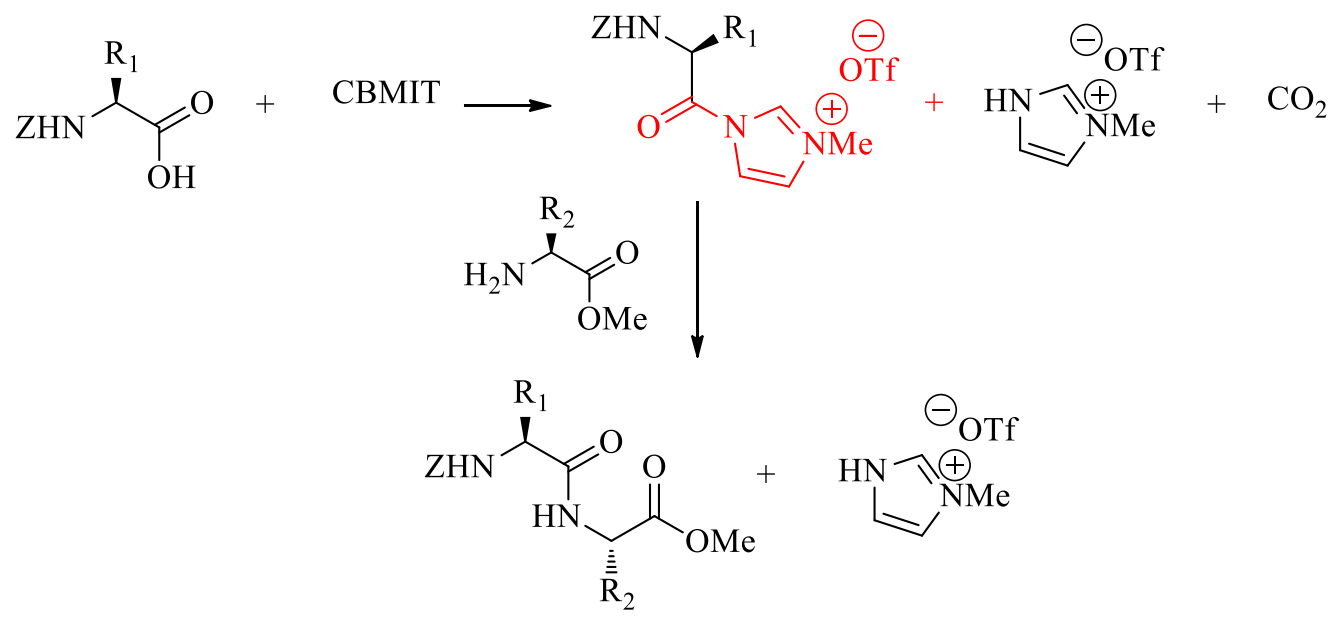

Scheme 44. Activation and coupling with CBMIT. 
A novel 1H-benzimidazolium- type peptide- coupling reagent, 2-chloro-1,3-dimethyl-1Hbenzimidazolium hexafluorophosphate (CMBI) was designed, synthesized, and shown to be efficient for the formation of sterically hindered amide and ester bonds. The mechanism of the CMDI mediated coupling is similar to the one described in Scheme 43. The carboxylate anion attacks the carbocation of the CMBI molecule to form an unstable acyloxybenzimidazolium intermediate, which in turn reacts with the amino component to give the product with the release of 1,3-dimethyl-2-benzimidazolidinone (Scheme 45).

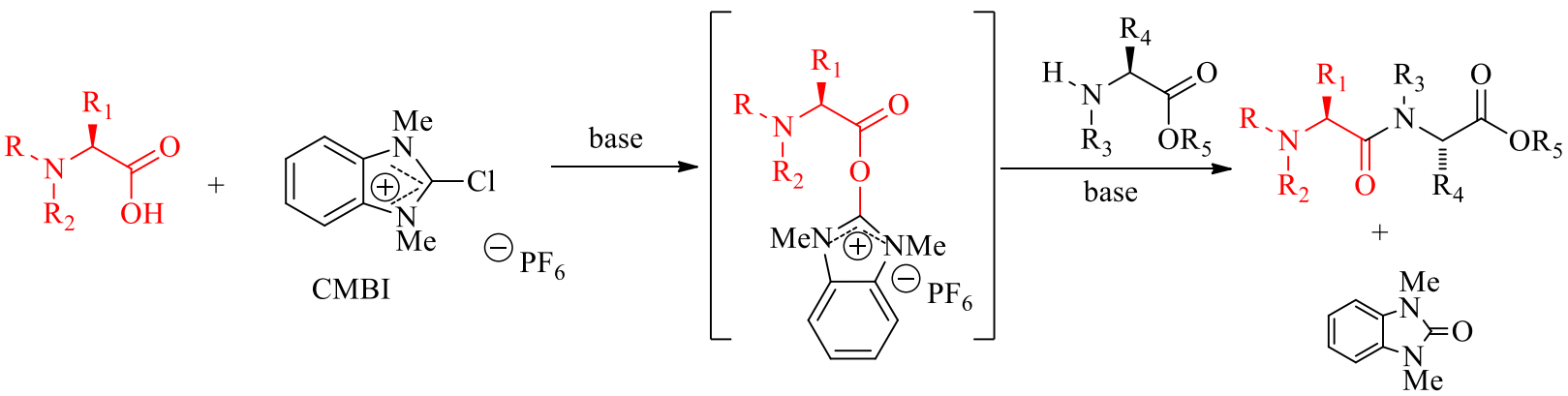

Scheme 45. CMBI mediated coupling.

Alternatively, the intermediate acyloxybenzimidazolium may be converted into the acid chloride, which could be converted into the desired peptide by aminolysis. Small amounts of a symmetric anhydride and 5(4H)-oxazolone derived from the $\alpha$-amino acid could also be generated and act as less reactive intermediates during coupling. The 5(4H)-oxazolone is prone to epimerization due to tautomerization or enolization under basic reaction conditions.

\subsection{Thiouronium salts}

A number of thio-uronium salts was prepared as reagents for peptide synthesis in solution and in the solid-phase. ${ }^{73,53}$ S-(1-oxido-2-pyridinyl)-1,1,3,3-tetramethylthiouronium hexafluorophosphate (HOTT) and tetrafluoroborate (TOTT) and S-(1-oxido-2-pyridinyl)-1,3-dimethyl-1,3trimethylenethiouronium hexafluorophosphate (HODT) and tetrafluoroborate (DODT) were prepared from 2-mercaptopyridine-1-oxide and 1,1,3,3-tetramethylurea (TMU) or 1,3dimethylpropyleneurea (DMPU). Their structures are shown in Figure 38.
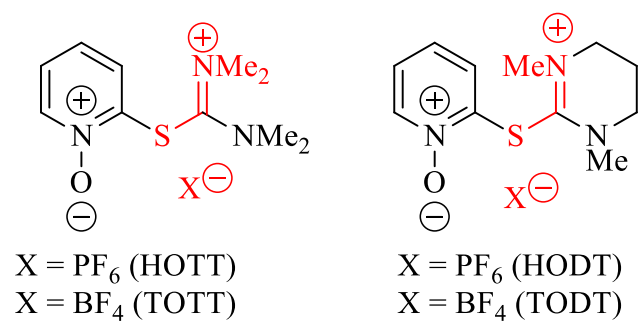

Figure 38. Structures of thiouronium salts derived from 2-mercaptopyridine-1-oxide. 
The thiouronium tetrafluoroborate (TODT) afforded higher yields than the thiouronium hexafluorophosphate (HODT). Racemization was higher with the DMPU- derived reagents than with the corresponding TMU- derivatives. The addition of 2-mercaptopyridine-1-oxide to the thiouronium salts improves the final yields and lowers the extent of racemization.

\section{8. (Chlorophenylthiomethylene)dimethylammonium chloride (CPMA)}

The reagent (chlorophenylthiomethylene)dimethylammonium chloride (CPMA) was first described to be used for the selective chlorination of primary hydroxyl groups. It was later found to be an efficient agent for the formation of esters and amides from carboxylic acids. ${ }^{74}$ It is compatible with substrates that are sensitive to basic conditions, or oxophilic reagents. The reaction proceeds without racemization of chiral centers in carboxylic acids. The reaction may be carried out at $0{ }^{\circ} \mathrm{C}$ in methylene chloride and in the presence of DIEA (Scheme 46).
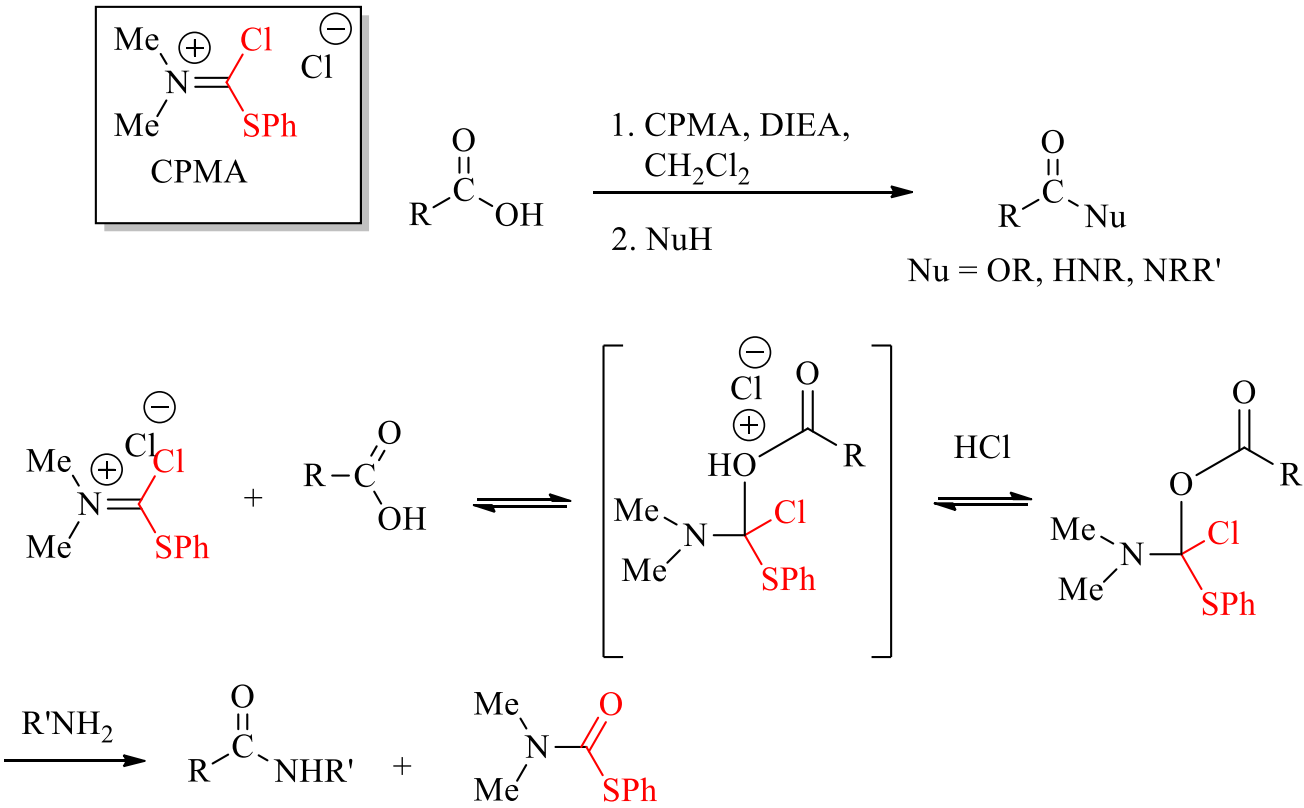

Scheme 46. Use and reactivity of CPMA.

\subsection{2,4-Bis(ethylsulfonyl)-1-naphthol (BMSN)}

2,4-Bis-(ethylsulfonyl)-1-naphthol (BMSN) (Figure 39) was tested as a carboxyl- activating agent for peptide bond formation. The N-protected amino acid esters of BMSN in the presence of triethylamine were suitable O-acylating agents for the formation of peptide bonds. The ultraviolet absorption allows the reaction to be followed by chromatography, and the by-product of the reaction, the disulfone, is easily removed by extraction with dilute sodium hydroxide. ${ }^{75}$ 
<smiles>[R]C(=O)Oc1c(OC)cc([S+]([O-])(O)OC)c2ccccc12</smiles>

BMSN active ester intermediate

Figure 39. 2,4-Bis(ethylsulfonyl)-1-naphthol (BMSN) intermediate.

\subsection{Bis $\alpha, \alpha-[$ (Trifluoromethyl)benzyloxy]diphenylsulfur (BTBDS)}

Investigations of sulfurane- based coupling agents showed that bis- $\alpha, \alpha-$ [(trifluoromethyl)benzyloxy]diphenylsulfur (BTBDS) facilitated rapid amide bond formation between $\mathrm{N}^{\alpha}$-urethane protected L-amino acids and L-phenylalanine ethyl ester, in the absence of bases, and in excellent yields with no detectable racemization. ${ }^{76}$ This research showed that sulfuranes can be useful in the coupling of racemization-sensitive systems. When compared with the uronium salts HBTU and HATU, it was shown that in the absence of bases, BTBDSmediated coupling gave better results (Scheme 47).

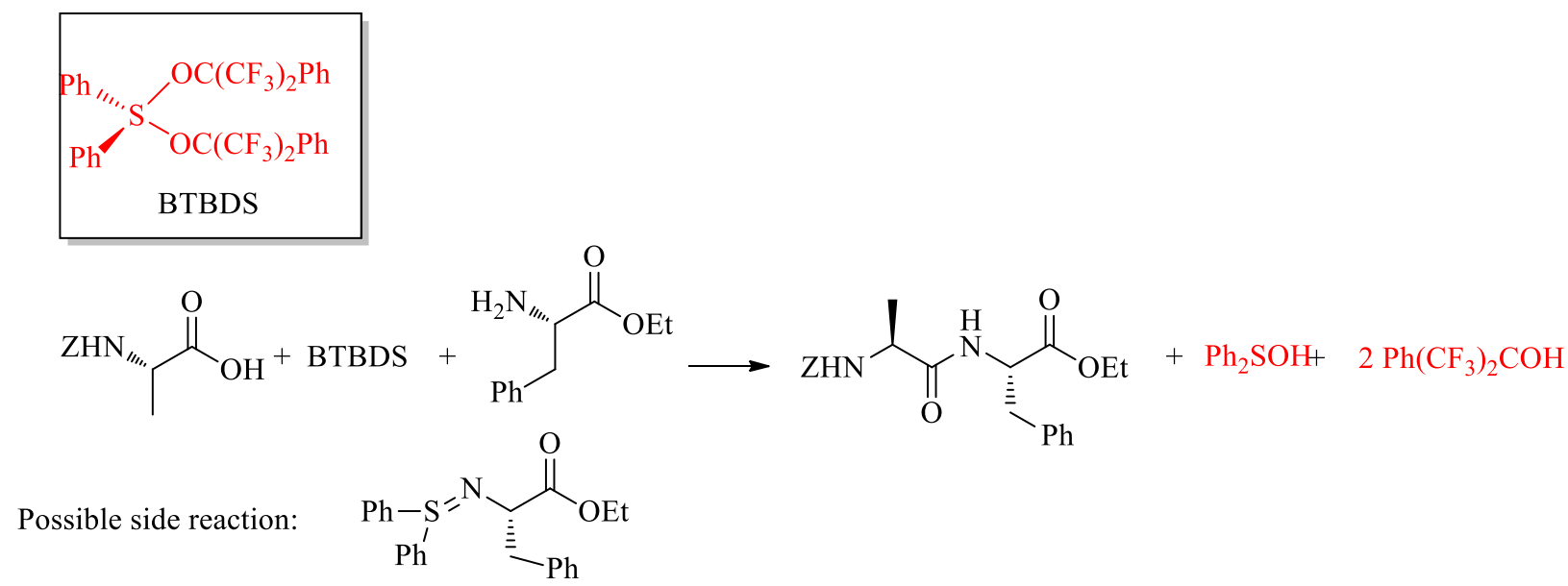

Scheme 47. Coupling with BTBDS.

\subsection{Tantalum pentachloride}

The observation that metal carboxylates may be considered formal analogs of organic esters led to the possibility of using metal carboxylates as acylating agents. The high positive charge of the metal center should promote nucleophilic attack at the carbonyl group. $\pi$-Acidic, oxophilic metal centers should favor attack on the carbonyl group. It was found that carboxylates of $\operatorname{Zr}(\mathrm{IV})$, $\mathrm{Ti}(\mathrm{IV})$, and $\mathrm{Ta}(\mathrm{V})$ did react readily with amines. The most active carboxylates were those of $\mathrm{Ta}(\mathrm{V})$. When chiral carboxylates were bound to $\mathrm{CpTa}(\mathrm{V}) \mathrm{Cl}_{3}$, their reactions with chiral amines at low temperature resulted in no racemization. ${ }^{77}$ As $N$-methylated amino acids represent one of 
the most difficult substrates in coupling reactions, the high reactivity of $\mathrm{Ta}(\mathrm{V})$ carboxylate might mediate substitution with hindered nucleophiles or hindered carboxylates under moderate conditions such as in toluene at room temperature. $\mathrm{TaCl}_{5}$ appears to have significant promise as an acylating agent for the synthesis of bulky amides.

This method is simple and convenient and utilizes a commercially available, inexpensive reagent with high retention of stereochemistry (Scheme 48).

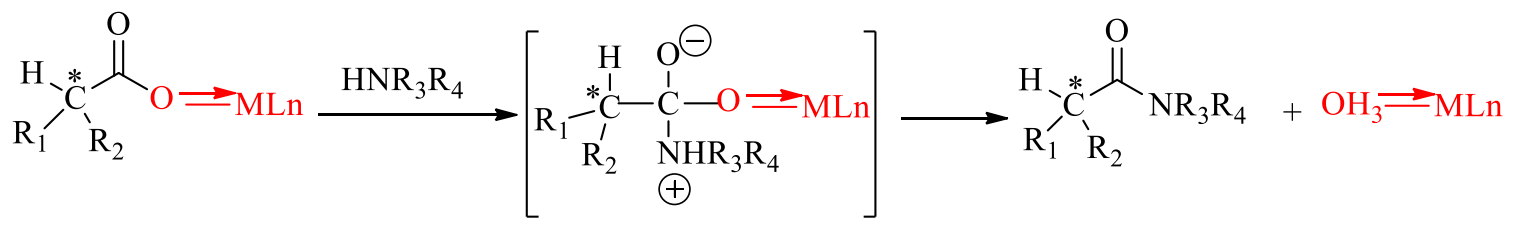

Scheme 48. Amide formation with metal carboxylates.

\subsection{2. $\mathrm{N}$-Alkylhydroxylamines and $\alpha$-keto acids}

Although most intermolecular amide syntheses involve addition-elimination reactions of activated carboxylates, a novel approach to amide synthesis is the decarboxylative condensation of $\mathrm{N}$-alkylhydroxylamines and $\alpha$-keto carboxylic acids (Scheme 49). The process occurs in protic or aprotic solvents, tolerates unprotected functional groups, and produces only water and carbon dioxide as byproducts. ${ }^{78,79}$ Other alternatives have been reported. ${ }^{80,81}$

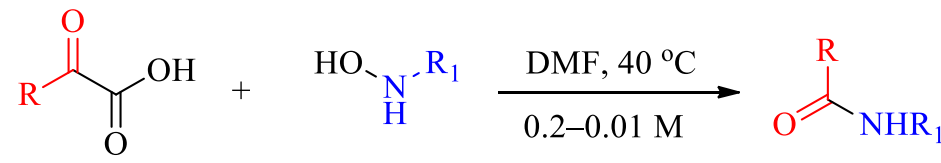

Scheme 49. Reaction of $\alpha$-keto acids with $\mathrm{N}$-alkylhydroxylamines.

\section{Chemical Ligation}

Total chemical synthesis of a protein overcomes the limitations of molecular biology and allows to effect any desired change in the structure of the protein. Chemical synthesis of proteins is crucial to studies of the protein function, and as a result has been the subject of many investigations. Effective chemical synthesis of proteins must satisfy many requirements. Modern chemical protein synthesis requires a convergent strategy and unprotected peptide segments. The chemoselective condensation of unprotected peptides in the total synthesis of protein molecules was first reported in $1992 .^{82}$

Chemical ligation is the chemoselective condensation of two unprotected peptides to give a unique covalent polypeptide product. The initial studies of ligation chemistry employed a peptide thiocarbonate and a bromoacetyl peptide, in aqueous solution and low $\mathrm{pH}$, to give a thioester- 
containing peptide. Another useful ligation was the oxime- forming ligation. Other ligation reactions forming non-native covalent links between two unprotected peptides are known.

The use of synthetic proteins containing non-native structures stimulated an extension of the original ligation chemistry. In this modification (Figure 40), the nucleophilic thioester-forming ligation reaction would use a peptide in which an additional methylene group was introduced in the bromoacyl moiety of the peptide that also contained an amino group. The initial thioesterlinked product would be set up for an intramolecular nucleophilic attack on the newly formed thioester functionality by the amino group, and the product resulting from this $S$ - to $N$ - acyl shift would be an amide-linked peptide (native peptide bond).

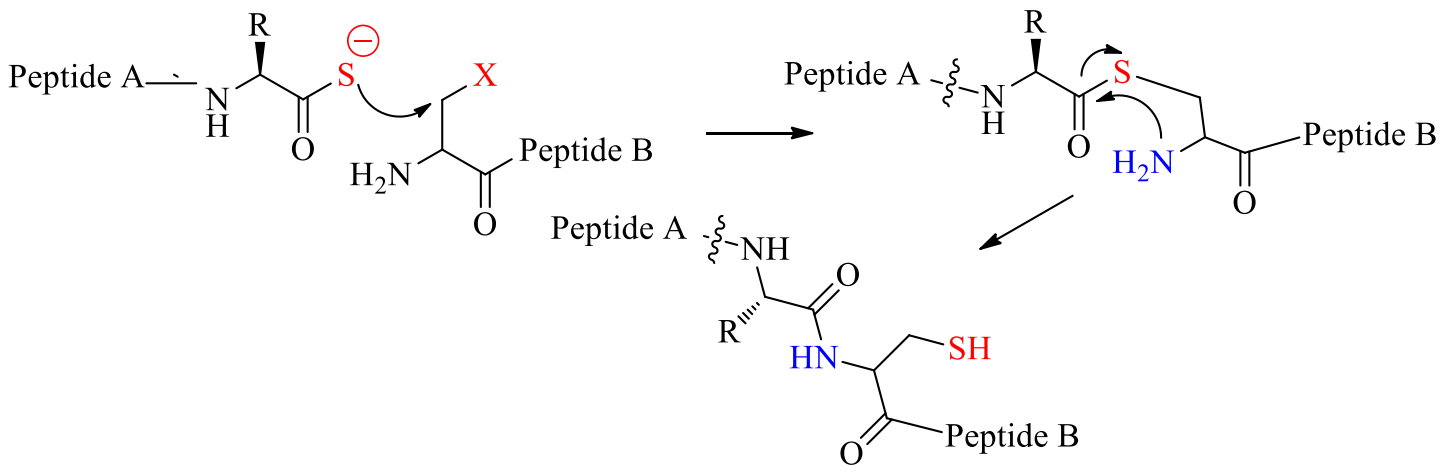

Figure 40. Modification of the original ligation chemistry (native chemical ligation).

\subsection{Native chemical ligation (NCL) approaches}

The chemistry of native chemical ligation (NCL) is now well established, and has recently been reviewed. ${ }^{83-86}$ While a review of the field is beyond the scope of this report, the importance of the process merits a brief discussion of the chemistry involved. NCL is one of the few nonenzymatic reactions that can join two unprotected peptide segments to generate a ligated peptide/protein product with a native peptide bond at the reaction site. The selective formation of an amide bond in the presence of unprotected functional groups is a challenging problem in peptide chemistry.

The possibility of generating an amide bond in aqueous solution through an intramolecular acyl shift was first reported by Wieland. ${ }^{87}$ This principle was used much later to develop the first ligation methodology for coupling two peptide fragments and was described as "prior thiol capture strategy". ${ }^{88,89}$ Extensive research into the design of the best template led to a benzofuran derivative that was used to study the kinetics of the acyl transfer (Figure 41). ${ }^{90}$ Peptide bond formation was shown to be very sensitive to steric interactions. 


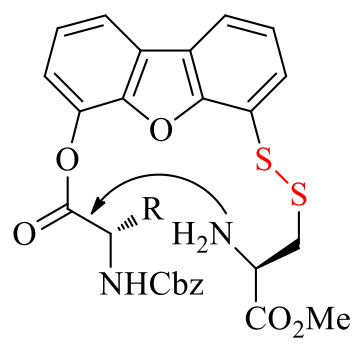

Figure 41. Kemp template.

The reaction involved in native chemical ligation is chemoselective, and involves a peptide fragment with a $\mathrm{N}$-terminal cysteine residue and a second peptide fragment containing a $\mathrm{C}$ terminal thioester group. The essence of the reaction is the trans-thio esterification step between the thioester of one peptide and the sulfhydryl group from the N-terminal cysteine residue in the other to generate a ligated thioester intermediate, which undergoes spontaneous $\mathrm{S}$ - to $\mathrm{N}$ - acyl rearrangement through a favorable five-membered ring to give the product (Scheme 50). ${ }^{91}$

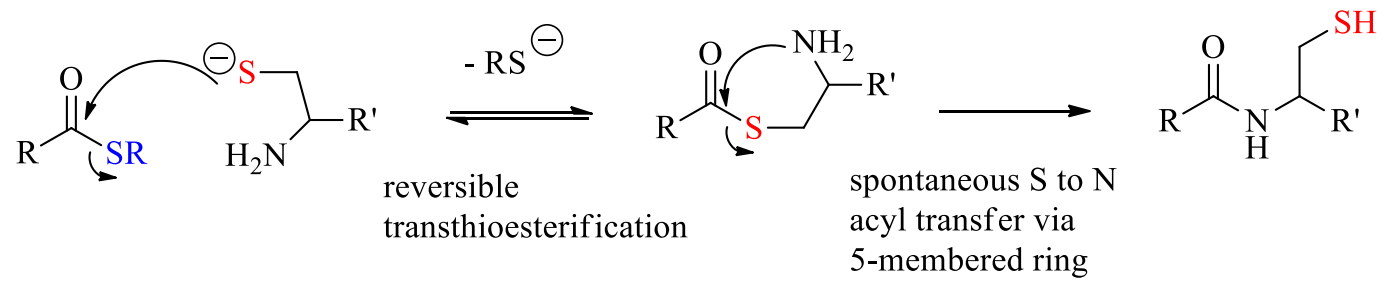

Scheme 50. Native chemical ligation.

Quinolinium thioester salts offer potential in peptide formation. ${ }^{92}$ The principle of native chemical ligation is to bring the coupling sites of two unprotected peptide fragments in close proximity. The ligated product undergoes acyl transfer to provide the peptide bond at the ligation junction. Templates for developing new amine capture strategies are desirable. To achieve in one single step the acyl transfer and release of the template is an improvement on other ligation processes. This strategy involves amine capture prior to peptide bond formation and is illustrated in Scheme 51. 


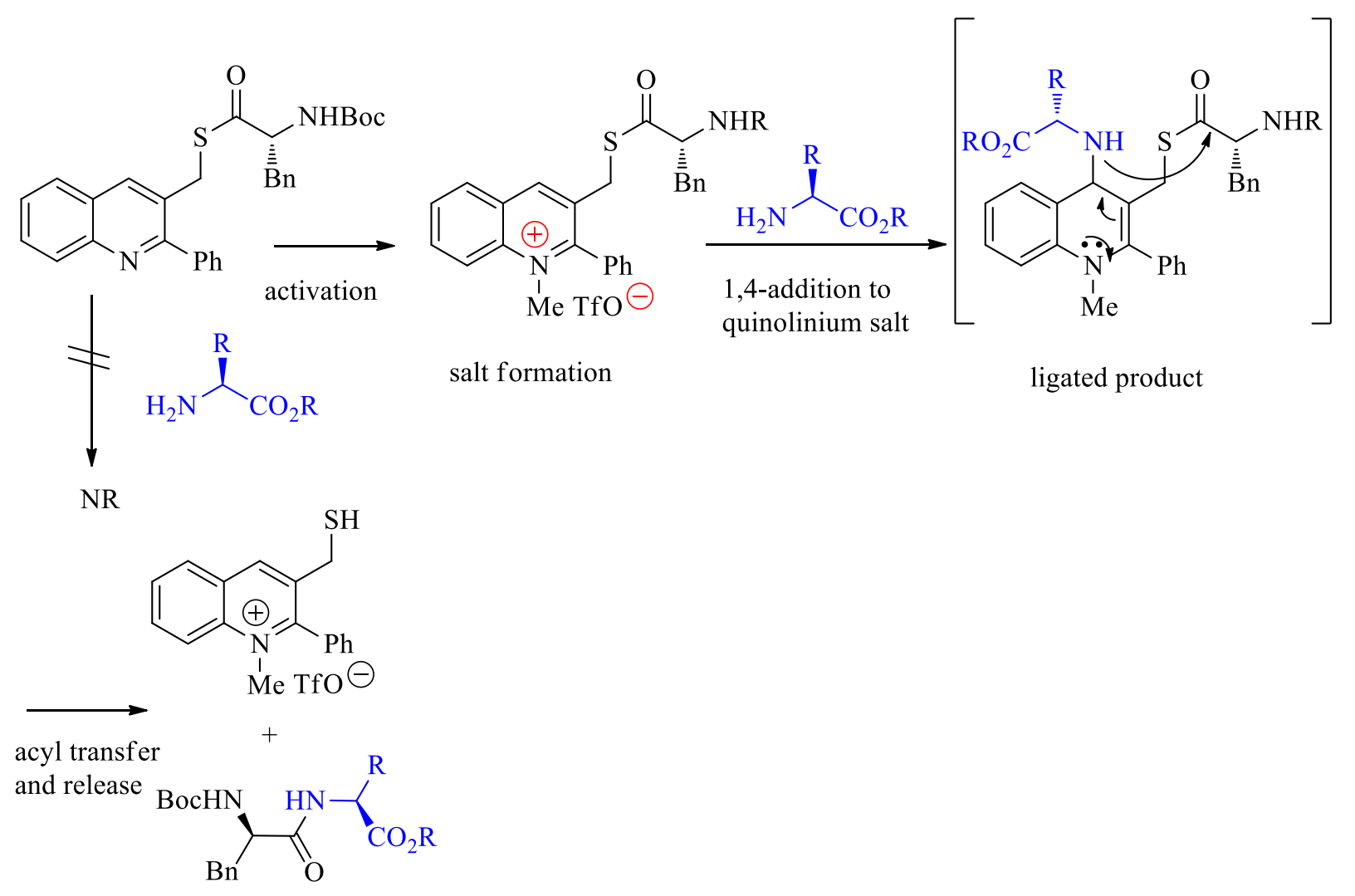

Scheme 51. Amine capture strategy by means of a quinolinium thioester salt.

In principle, reversible attachment of the functional equivalent of a cysteine side chain onto the N-terminus of a peptide would provide a universal ligation chemistry. This approach has been attempted using a cleavable amino-oxy- linked ethanol side chain but the slow rearrangement of the thioester intermediate through a less favorable six-membered ring onto an aminoxy group limited its application..$^{93}$

As the chemical ligation involves coupling of the $\mathrm{C}$-terminus of a thioester to the $\mathrm{N}$-terminal cysteine residue, the limiting phase of the interaction is the intermolecular trans-esterification reaction to generate a unimolecular intermediate that is positioned to undergo the subsequent $\mathrm{S}$ to $\mathrm{N}$ - acyl transfer. In addition, large protected peptides are hard to handle in solution, the cleavage of protecting groups often require harsh conditions and afford poor yields, and the activation of the desired peptide thio-ester is prone to epimerization at the C-terminal alphacarbon. Several strategies have evolved to broaden the scope of the reaction.

The synthesis of peptide and protein thio-esters that can take part in NCL reactions has been examined using the transient production of another thio-ester that can then be ligated to the desired peptide to effect the NCL reaction. ${ }^{94,95}$

The chemoselective coupling of two protein fragments to give a native peptide bond at the point of ligation in aqueous solution and in the absence of protecting groups is a powerful technology which is now at the forefront of protein synthesis. As expected, the methodology is 
rapidly being changed by evolving strategies that overcome some of the limitations of the process. 96

Several strategies have been tried, and based on their observations it was suggested that a $N$ linked 2-mercaptobenzyl group could form the basis of a new peptide ligation chemistry (Figure 42). ${ }^{97}$

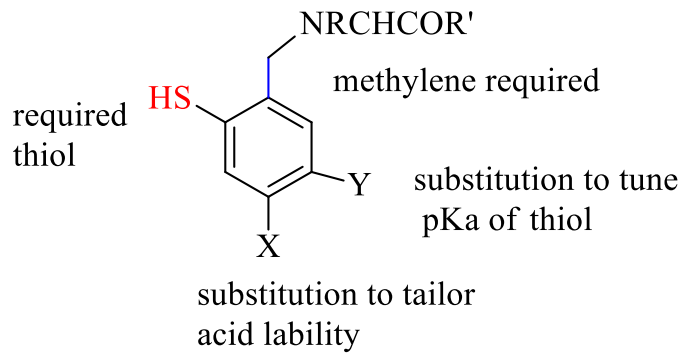

Figure 42. Design of a 2-mercaptobenzylamino tether with potential for extended chemical ligation.

The model requires tailoring the mercaptobenzyl skeleton for lability. A reversible group for assisting chemoselective ligation would deliver highly flexible routes for protein synthesis.

Nevertheless new techniques are promising, and offer rapid access to peptides that were previously inaccessible. ${ }^{84}$ The process has been applied to the total chemical synthesis of enzymes. A sortase-mediated ligation of proteins to both peptides and non- peptides was demonstrated in vitro to be a new method for protein engineering. ${ }^{98}$

As mentioned earlier (3.1), azides are involved in the synthesis of proteins by native chemical ligation (NCL). A modification produced the amide bond between the two coupling partners without an intervening triaryl phosphine oxide and is referred to as the "traceless" Staudinger reaction. ${ }^{99}$ Phosphines incorporating two elements were designed for this purpose. The acyl component destined for the amide bond was attached to the aryl ring via a cleavable linkage. The nucleophilic nitrogen atom of the aza-ylide attacks the carbonyl group, displacing the cleavable linkage and attached phosphonium group. Hydrolysis of the rearranged adduct produces the amide bond and liberates a phosphine oxide. A phenyl ester and $N$-acylimidazole phosphines were the optimal substrates. Two aromatic substituents were included to impart stability toward oxygen (Figure 43). 

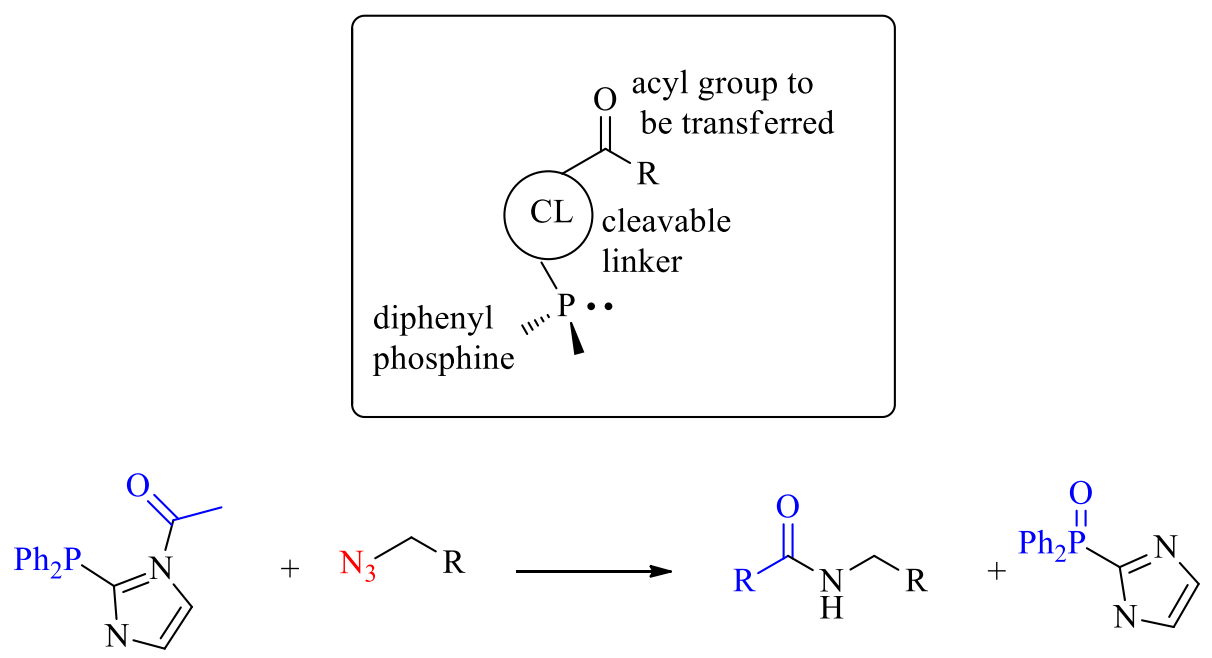

Figure 43. Design of a phosphine reagent for the selective formation of an amide.

A limitation of the NCL method was the requirement for an N-terminal cysteine, and as a result the need for multiple ligation and deprotection cycles to assemble long peptide sequences. This problem was addressed by the development of thiol auxiliaries which when attached to the $\mathrm{N}$-terminus of a synthetic peptide facilitated ligation and could easily be removed after completion of the coupling. Some of these auxiliaries are shown in Figure 44. ${ }^{97,}$ 100, 101<smiles>[R]C(NC(CS)c1ccc(OC)cc1OC)C(=O)O</smiles><smiles>[R]C(NCc1c(S)cc(OC)c(OC)c1OC)C(C)=O</smiles>

Figure 44. Examples of chiral auxiliaries.

Considerable reports have dealt with their scope, transition state and ease of cleavage. Although the thiol auxiliaries methodology was applicable to synthetic peptides, the introduction of an auxiliary to the $\mathrm{N}$-terminus of an expressed protein required a different approach.

Another limitation of the NCL method is that it is not totally convergent, and this issue has been addressed by several workers. The Kent group has focused on the differences in reactivity between alkyl- and aryl- thio-esters and showed that peptide components containing an $\mathrm{N}$ terminal cysteine residue and an alkyl thio-ester fragment reacted with an aryl thio-ester fragment, the ligation proceeded readily leaving the alkyl thio-ester intact. Aryl thio-esters may be prepared from alkyl thio-esters upon exposure to thiophenol at $\mathrm{pH} 6.8 .^{102}$

The Danishefsky group reported a major simplification in the field of native chemical ligation. ${ }^{103}$ In the Kent method, the rate- limiting phase of the sequence is the intermolecular 
trans- thio-esterification which generates a unimolecular intermediate well positioned to undergo sulfur- to nitrogen- acyl transfer. A variation of the native chemical ligation allowed for the merger of glycopeptide fragments through the use of a relatively inert C-terminal orthothiophenolic ester obtained from cleavage of the corresponding disulfide. ${ }^{104}$ Upon cleavage of the $\mathrm{S}-\mathrm{S}$ bond, the inert $\mathrm{C}$-terminal ortho-thiophenolic ester undergoes intramolecular $\mathrm{O}$ - to $\mathrm{S}$ transfer. The intermediate thio-ester is then trapped intermolecularly by the N-terminal cysteine residue of its coupling partner. A final $\mathrm{S}$ to $\mathrm{N}$ acyl transfer affords the desired product.

The limitation of this approach is that ligation at sterically hindered, beta-branched Cterminal amino acids is extremely slow. Therefore, improved procedures were devised using various approaches. As the rate-limiting $\mathrm{S}$ - to $\mathrm{S}$ - acyl transfer was dependent on the steric hindrance at the C-terminal amino acid, the possibility of finding a method that would avoid the thio-ester was sought. Using an active $p$-nitro-phenyl ester that has been used in direct peptide coupling, ${ }^{5}$ a major simplification in the field was accomplished showing that a thio-ester donor was not required. Proper balancing of acyl donor potential with substrate stability allowed the use of oxo-esters rather than thio- esters. ${ }^{103}$ A general picture of both processes is shown in Figure 45.

A
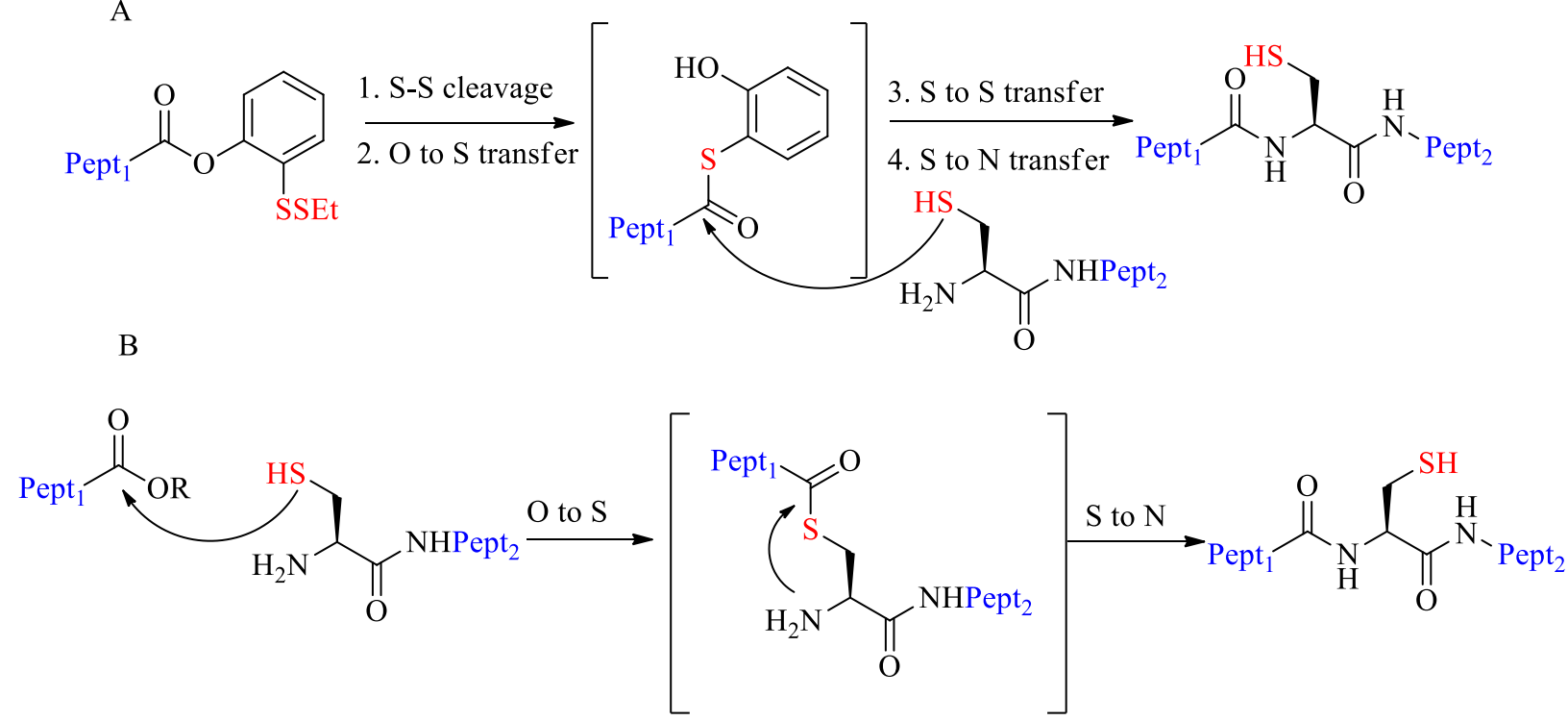

Figure 45. A. Phenolic ester variation of NCL. B. Proposed oxo-ester variation of NCL.

$\mathrm{N}$-Acyl activation has been accomplished with $\mathrm{N}$-acyl- ureas that can undergo rapid thiolysis to yield thio-ester peptides under standard native chemical ligation conditions. ${ }^{105}$

The direct synthesis of peptide thio-ester precursors assembles the target peptide sequence on a solid phase as an $o$-amino-anilide, whose functionality is then transformed into a mildly activated $\mathrm{N}$-acylbenzimidazolinone via cyclization through a carbonyl donor, $p$-nitrophenyl chloroformate. After global deprotection and cleavage from the resin by standard methods, the 
peptide $\mathrm{N}$-acylbenzimidazolinone is purified and subjected to thiolysis in the presence of thiol additives to yield the required peptide thio-ester. Thio-ester exchange with its reactive partner is followed with the intramolecular S- to N-acyl transfer to afford the desired product (Scheme 52). The C-terminus already activated as the $\mathrm{N}$-acylbenzimidazolinone obviates post-cleavage reaction procedures. ${ }^{105}$
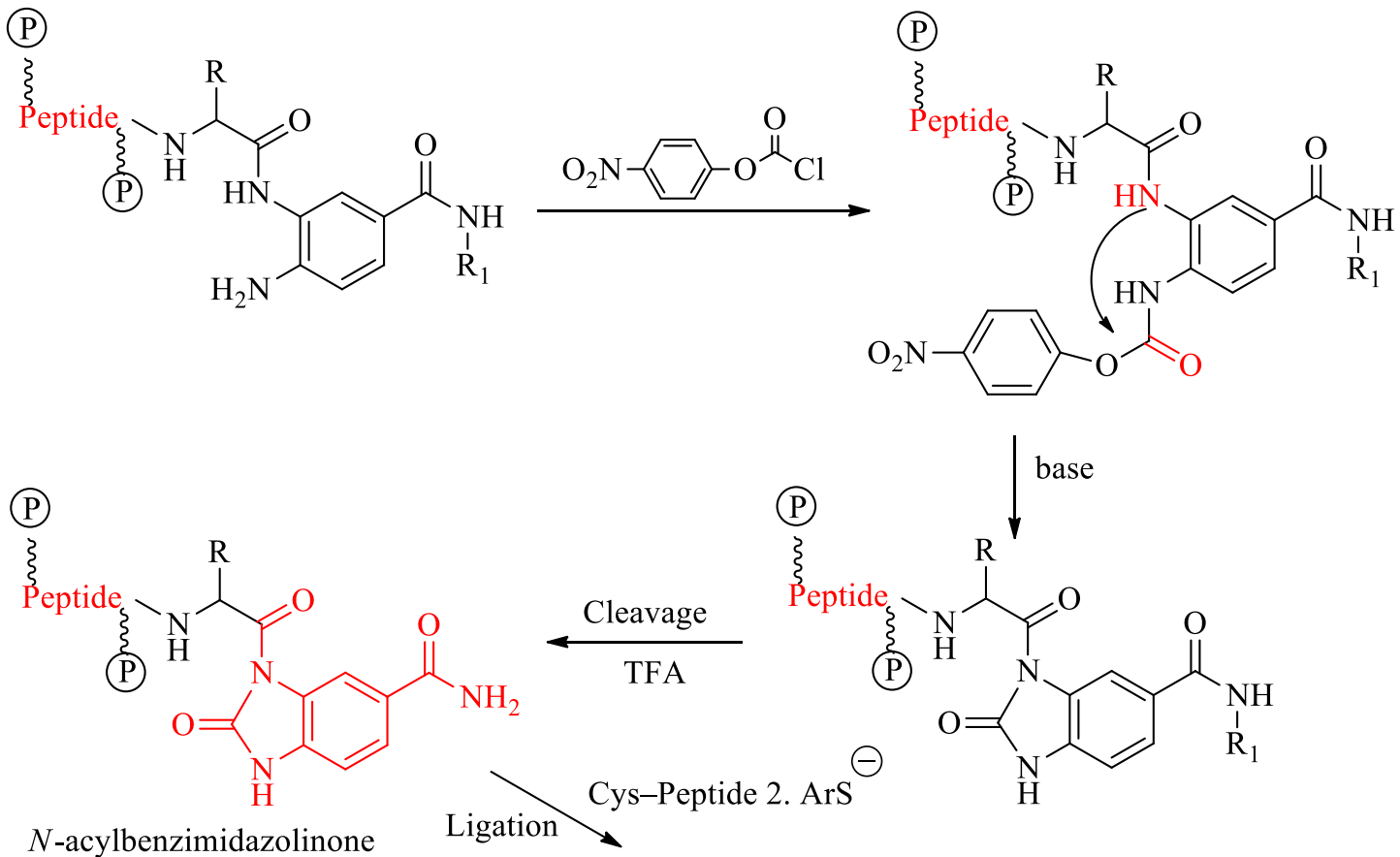

Target Peptide

Scheme 52. Preparation of peptide $N$-acylbenzimidazolinone.

A different way to circumvent the need for a cysteine residue is to use an amino acid surrogate containing a thiol moiety. After ligation the surrogate is converted into the desired amino acid. This operation has been accomplished in several ways: converting cysteine into serine; homocysteine coupling and subsequent methylation; installing a sulfhydryl group temporarily at a non-cysteine site; or using a gamma-thiol -valine as a valine surrogate. ${ }^{106}$

The $\alpha$-keto acid-hydroxylamine amide-forming ligation reaction that allows the two unprotected molecules to form an amide bond (4.1) present a synthetic challenge when applied to peptide chains that contain the requisite functional groups. A new $N$-sulfonyloxaziridine has been found to selectively oxidize primary amines to hydroxylamines for application to the synthesis of N-terminal peptide hydroxylamine for chemoselective ligation (Scheme 53). ${ }^{107}$

In addition, a general strategy for the preparation of C-terminal peptide $\alpha$-keto acids by solid phase peptide synthesis has been developed using a cyanosulfur-ylide based linker (Scheme 54). ${ }^{108}$ 
<smiles>O=Cc1ccccc1C12ON1S(=O)(=O)c1ccccc12</smiles>

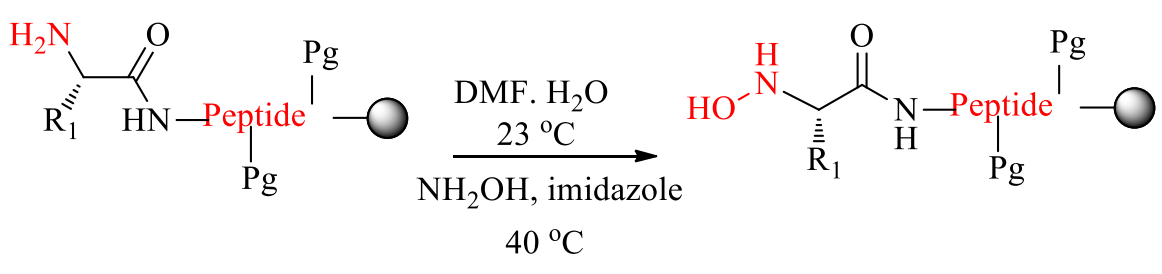

Scheme 53. Oxidation of a primary amine to a hydroxylamine.<smiles>[R7]C(CNP)C(=O)C(C#N)=S1CCCC1</smiles>

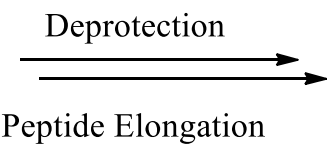

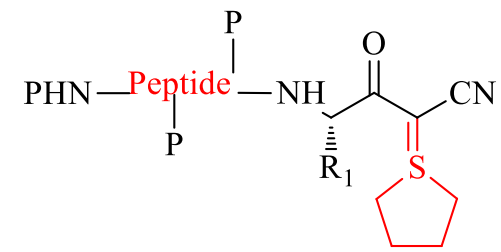

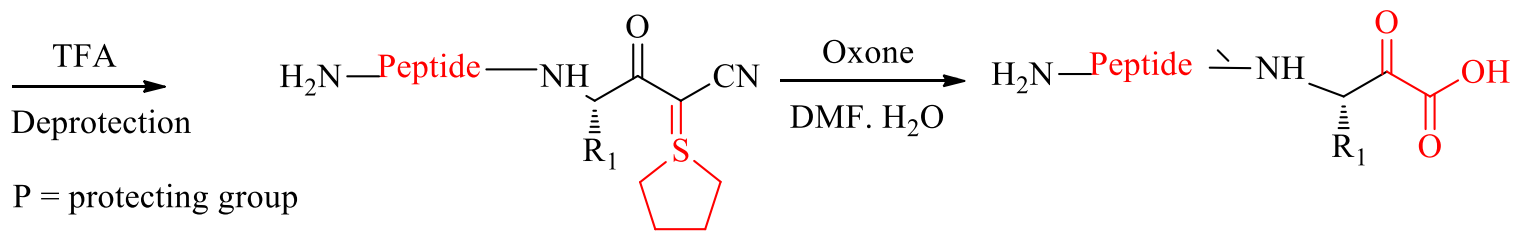

Scheme 54. Formation of a C-terminal peptide $\alpha$-keto acid.

Although both approaches need optimization they will enable further research and applications of the $\alpha$-keto acid-hydroxylamine amide-formation to complex peptide synthesis.

Peptides and proteins play a central role in numerous biological and physiological processes in living organisms. Understanding the control of the mechanisms that govern their properties at the molecular level is a challenging research topic. For this study the availability of large quantities of pure material is essential. Only chemical peptide synthesis will allow the production of large quantities of pure peptides and the production of unnatural amino acids that can be used in a variety of ways.

\section{Discussion}

Amide formation is the reaction involved in forming peptide bonds. Peptide bond formation was reported as early as 1881 by Curtius. ${ }^{109}$ Since the publication of glycylglycine by Emil Fischer in $1901,{ }^{110}$ peptide science has made amazing progress and it is now possible to synthesize rather long proteins. It may be seen from the previous description that a plethora of reagents and mechanisms is available for the formation of peptide bonds. The final choice rests on the substrate but a few generalizations emerge from the various investigations. 
Many coupling reactions are conducted under dry conditions. Purification and isolation of the activated acid-derivatives may be difficult, especially if they are unstable. In situ formation of the activated acid derivative is more convenient and advantageous, especially if the carboxylic acid is valuable. Reactions are usually conducted in less polar solvents, such as ethers, methylene chloride, tetrahydrofuran, and acetonitrile. In situ activation in water is practically limited to water-soluble carbodiimides.

The presence of side products is tied to the coupling methods. Efficient coupling reagents in combination with racemization suppressor additives and solvents of low dielectric constant should minimize the formation of epimers. Reducing the time of pre-activation of the carboxylic acid could also reduce racemization. Racemization is chromatographically difficult to detect because both products have similar chromatographic behavior. Aminium salts can form guanidino derivatives with free amino functions. Acids can terminate the peptide chain. Deprotection and cleavage may produce changes in the product, and appropriate scavengers should be used to avoid these pitfalls. Ureas from carbodiimides and aminium salts, phosphotriamides from phosphonium salts, coupling additives and scavengers used in the last step may be reaction by-products that will need to be removed.

For systems that undergo slow activation, sequential addition of more of the coupling reagent is advisable, since it may be hydrolyzed after a few minutes.

Intramolecular hydrogen bonds in a linear precursor may favor or disfavor the optimal conformation for cyclization. PyBroP was efficient in methylene chloride but not in DMF. Solvation of the linear precursor may favor an unfavorable conformation, while in methylene chloride or deuterated chloroform a bent conformation may be preferred. The proper turnconformation of a linear peptide in a dilute solution of an appropriate solvent may facilitate the macrocyclization.

Reagents containing imidazole, benzotriazoles and reagents with extra nitrogen in the ring have some sensitivity to friction that may produce burning or explosion. Also, certain coupling reagents and additives may show adverse effects such as contact dermatitis, skin irritation, and allergic reaction of the respiratory tract. ${ }^{45}$

\section{Conclusions}

The formation of amide bonds plays an important role in organic chemistry and biochemistry. Several cyclopeptides containing this moiety have unique biological properties and synthetic complexity. A plethora of peptides and proteins contain this bond and the synthesis of these products is increasingly important. A careful analysis of the mechanism involved in each method is essential for the optimization of the coupling step.

In addition, chemistry is of utmost importance in the study of biological processes. Chemical synthesis of large peptides by intermolecular coupling of smaller peptides using conventional peptide- bond- forming techniques did not realize its potential advantages until recently. Using 
an approach based on highly specific and efficient ligation of two peptide segments prior to the formation of the peptide bond, the two sub-units are ligated in such a way that the carboxyl terminus of one and the amino terminus of the other are close together so that a proximity-driven intramolecular acyl transfer can occur to form a new peptide bond.

To summarize, chemical ligation allows the covalent joining of unprotected peptides by the chemoselective reaction of unique, mutually reactive functional groups to give a product of defined covalent structure. Native chemical ligation extended the concept to form amides thereby creating a native peptide bond. ${ }^{111}$

Advances in the area of protein structure and function are based on strategies developed from a simple chemical reaction - amide bond formation - and illustrate the power of chemical science in elucidating protein-ligand interactions.

\section{Acknowledgements}

M.M.J. wishes to acknowledge her collaborators over the years for their contributions in this area, and the many dedicated researchers that have contributed to the evolution of simple amide bonds to protein engineering. We thank Dr. Michael Leonard for reading the manuscript and making useful suggestions.

\section{References}

1. Valeur, E.; Bradley, M. Chem. Soc. Rev. 2009, 38, 606.

2. Han, S.-Y.; Kim, Y.-A. Tetrahedron 2004, 60, 2447.

3. Montalbetti, C. A. G. N.; Falque, V. Tetrahedron 2005, 61, 10827.

4. Kemp, D. S. In The Peptides; Gross, E., Meienhofer, J. Eds. Ed.; Academic Press: New York, 1979; pp 317-378.

5. Bodansky, M. Principles of Peptide Synthesis; Springer-Verlag: New York, 1984.

6. Davies, J. S.; Howe, J.; Jayatilake, J.; Riley, T. Lett. Pept. Sci. 1997, 4, 441.

7. Kemp, D. S. Racemization in Peptide Synthesis; Academic Press, 1979; Vol. 1, Ch. 7.

8. Chincilla, R.; Dodsworth, D. J.; Nấjera, C.; Soriano, J. M. Tetrahedron Lett. 2001, 42, 4487.

9. König, W.; Geiger, R. Chem. Ber. 1970, 103, 788.

10. Carpino, L. A. J. Am. Chem. Soc. 1993, 115, 4397.

11. Sabatino, G.; Mulinacci, B.; Alcaro, M. C.; Chelli, M.; Rovero, P.; Papini, A. M. Letters in Peptide Science 2003, 9, 119.

12. Carpino, L., A.; Xia, J.; El-Faham, A. J. Org. Chem. 2004, 69, 54.

13. Malow, M.; Wehrstedt, K. D.; Neuenfeld, S. Tetrahedron Lett. 2007, 48, 1233.

14. Wehrstedt, K. D.; Wandrey, P. A.; Heitkamp, D. J. Hazard. Mater. 2005 126, 1. 
15. Jones, J. H. The Formation of Peptide Bonds:A General Survey; Academic Press, Inc., 1979; Vol. 1.

16. Shioiri, T.; Ninomiya, K.; Yamada, S. J. Am.Chem. Soc. 1972, 94, 6203.

17. Hai, S. M.; Lwowski, W. J. Org. Chem. 1973, 38, 2442.

18. Vandensavel, J.-M.; Smets, G.; L'Abbe, G. J. Org. Chem. 1973, 38, 675.

19. O'Brien, P. M.; Sliskovic, D. R.; Blankley, C. J.; Roth, B. D.; Wilson, M. W.; Hamelehle, K. L.; Kraus, B. R.; Stanfield, R. L. J. Med. Chem. 1994, 37, 1810.

20. Nilsson, B. L.; Hondal, R. J.; Soellner, M. B.; Raines, R. T. J. Am. Chem. Soc. 2003, 125, 5268.

21. Leonard, M. S.; Joullié, M. M. Encyclopedia of Reagents for Organic Synthesis, 2003; Vol. DOI: 10.1002/047084289X.rn00055.

22. Dudash, J., Jr.; Jiang, J.; Mayer, S. C.; Joullie, M. M. Synth. Commun. 1993, 23, 349.

23. Jiang, J.; Li, W. R.; Przeslawski, R. M.; Joullie, M. M. Tetrahedron Lett. 1993, 34, 6705.

24. Davis, A. P.; Walsh, J. J. Tetrahedron Lett. 1994, 35, 4865.

25. Carpino, L. A.; Beyermann, M.; Wenschuh, H.; Bienert, M. Acc. Chem. Res. 1996, 29, 268.

26. Schmidt, U.; Kroner, M.; Beutler, U. Synthesis 1988, 475.

27. Savdra, J.; Chertanova, L.; Wakselman, M. Tetrahedron 1994, 50, 5309.

28. Carpino, L. A.; El-Faham, A. J. Am. Chem. Soc. 1995, 117, 5401.

29. Carpino, L. A.; Ionescu, D.; El-Faham, A.; Beyermann, M.; Henklein, P.; Hanay, C. W., H.; Bienert, M. Org. Lett. 2003, 5, 975.

30. Belleau, B.; Malek, G. J. Am. Chem. Soc. 1968, 90.

31. Jaquadi, M.; Selve, C.; Dormoy, J. R.; Castro, B. Bull. Soc. Chim. Fr. 1984, 409.

32. Cabaret, D.; Wakselman, M. Tetrahedron Lett. 1994, 35, 9561.

33. Bosch, I.; Urpi, F.; Vilarrasa, J. J. Chem. Soc., Chem. Commun. 1995, 91.

34. Fuller, W. D.; Cohen, M. P.; Shabankareh, M.; Blair, R. K. J. Am. Chem. Soc. 1990, 112, 7414.

35. Sheehan, J. C.; Hess, G., P. J. Am. Chem. Soc. 1955, 77, 1067.

36. Benoiton, N. L. Biopolymers 1996, 40, 245.

37. Benoiton, N. L.; Chen, F. M. F. J. Chem.Soc. Chem. Comm. 1981, 543.

38. Dalpozzo, R.; Bartoli, G.; Bosco, M.; Melchiorre, P.; Sambri, L. Curr. Org. Synth. 2009, 6, 79.

39. Agami, C.; Couty, F. Tetrahedron 2002, 58, 2701.

40. Bodlaender, P.; Feinstein, G.; Shaw, E. Biochemistry 1969, 8, 4941.

41. Castro, B.; Dormoy, J. R. Tetrahedron Lett. 1972, 13, 4747.

42. Castro, B.; Dormoy, J. R. Tetrahedron Lett. 1973, 14, 3243.

43. Castro, B.; Dormoy, J. R.; Evin, G.; Selve, C. Tetrahedron Lett. 1975, 1219.

44. Frérot, E.; Coste, J.; Pantaloni, A.; Dufour, M.-N.; Jouin, P. Tetrahedron 1991, 259.

45. Marder, O.; Albericio, F. Chim. Oggi 2003, 35.

46. Li, P.; Xu, J.-C. Tetrahedron 2000, 56, 4437.

47. Li, P.; Xu, J. C. J. Org. Chem. 2000, 65, 2951. 
48. Ogura, H.; Nagai, S.; Takeda, K. Tetrahedron Lett. 1980, 21, 1467.

49. Goodman, M.; Zapf, C.; Rew, Y. Biopolymers 2001, 60, 229.

50. Li, H.; Jiang, X.; Ye, Y.; Fan, C.; Romoff, T.; Goodman, M. Org. Lett. 1999, 1, 91.

51. Ye, Y.; Li, H.; Jiang, X. Biopolymers 2005, 80, 172.

52. Van Der Auwera, C.; Anteunis, M. J. O. Int. J. Peptide Protein Res. 1987, 29, 574.

53. Bailen, M. A.; Cinchilla, R.; Dodsworth, D. J.; Najera, C. J. Org. Chem. 1999, 64, 8936.

54. Chesworth, R.; Zawistoski, M. P.; Lefker, B. A.; Cameron, K. O.; Day, R. F.; Mangano, F. M.; Rosati, R. L.; Colella, S.; Petersen, D. N.; Brault, A.; Lu, B.; Pan, L. C.; Perry, P.; Ng, O.; Castleberry, T. A.; Owen, T. A.; Brown, T. A.; Thompson, D. D.; DaSilva-Jardine, P. Bioorg. Med. Chem. Lett. 2004, 14.

55. Hartung, J.; Schwarz, M. Synlett (Accounts and Rapid Communications in Synthetic Organic Chemistry) 2003, 3, 371.

56. Bofill, J. M.; Albericio, F. Tetrahedron Letters 1999, 40, 2641.

57. Abdelmoty, i.; Albericio, F.; Carpino, L. A.; Foxman, B. M.; Kates, S. A. Lett. Pept. Sci. 1994, $1,57$.

58. Albericio, F.; Bofill, J., M.; El-faham, A.; Kates, S., A. J. Org. Chem. 1998, 63, 9678.

59. Carpino, L. A.; El-Faham, A. A.; Albericio, F. J. Org. Chem, 1995, 60, 3561.

60. Knorr, R.; A, T.; Bannwarth, W.; Gilessen, D. Tetrahedron Lett. 1989, 30, 1927.

61. Carpino, L., A.; Xia, J.; Zhang, C.; El-Faham, A. J. Org. Chem. 2004, 69, 62.

62. Appel, R.; Glaesel, U. Chem. Ber. 1980, 113, 3511.

63. Yasuhara, T.; Nagaoka, Y.; Tomioka, K. J. Chem. Soc., Perkin Trans. 1 1999, 2233.

64. Yasuhara, T.; Nagaoka, Y.; Tomioka, K. J. Chem. Soc., Perkin Trans. 1 2000, 2901.

65. Schmidt, U.; Griesser, H. J. Chem. Soc. Chem. Commun. 1993, 1461.

66. Kunishima, M.; Kawachi, C.; Hioki, K.; Tani, S. Tetrahedron 2001, 57, 1551.

67. Kunishima, M.; Kawachi, C.; Morita, J.; Terao, K.; Iwasaki, F.; Tani, S. Tetrahedron 1999, $55,13159$.

68. Li, P.; Xu, C. Tetrahedron Lett. 1999, 40, 8301.

69. Akaji, K.; Aimoto, S. Tetrahedron 2001, 57, 1749.

70. Akaji, K.; Kurihama, N.; Kiso, Y. Tetrahedron Lett. 1994, 35, 3315.

71. Akaji, K.; Tamai, Y.; Kiso, Y. Tetrahedron 1997, 53, 567.

72. Saha, A. K.; Schultz, P.; Rapoport, H. J. Am. Chem. Soc. 1989, 111, 4856.

73. Albericio, F.; Bailen, M. A.; Chinchilla, R.; Dodsworth, D. J.; Najera, C. Tetrahedron 2001, 57, 9607.

74. Gomez, L.; Ngouela, S.; Gellibert, F.; Wagner, A.; Mioskowski, C. Tetrahedron Lett. 2002, $43,7597$.

75. Bereznak, J. F.; Joullié, M. M. Synth. Commun. 1989, 19, 3573.

76. Slaitas, A.; Yeheskiely, E. J. Peptide Res. 2002, 60, 283.

77. Fang, J. B.; Sanghi, R.; Kohn, J.; Goldman, A. S. Inorg. Chim. Acta 2004, 357, 2415.

78. Bode, J. W.; Fox, R. M.; Baucom, K. D. Angew. Chem. Int. Ed. 2006, 45, 1248.

79. Hackenberger, C. P. R.; Scharzer, D. Angew. Chem. Int. Ed. 2008, 47, 10030. 
80. Bode, J. W. Curr. Opin. Drug Discovery Development 2006, 9, 765.

81. Gunanathan, C.; Ben-David, Y.; Milstein, D. Science 2007, 317, 790.

82. Schnolzer, M.; Kent, S. B. H. Science 1992, 256, 221.

83. Anthony-Cahill, S. J.; Magliery, T. J. Curr. Pharm. Biotech. 2002, 3, 299.

84. Coltart, D. M. Tetrahedron 2000, 56, 3449.

85. Dawson, P. E.; Kent, S. B. H. Ann. Rev. Biochem. 2000, 69, 923.

86. Kent, S. B. H. Chem. Soc. Rev. 2009, 38, 338.

87. Wieland, T.; Bokelmann, E.; Bauer, L.; Lang, H. U.; Lau, H. Justus Liebigs Ann. Chem. 1953, 583, 129.

88. Kemp, D. S.; Galakatos, N. G.; Kerkman, D. J. Tetrahedron Lett. 1981, 22, 181.

89. Kemp, D. S.; Kerkman, D. J. Tetrahedron Lett. 1981, 22, 185.

90. Kemp, D. S.; Galakatos, N. G.; Dranginis, S.; Ashton, C.; Fotouhi, N.; Curran, T. P. J. Org. Chem. 1986, 51, 1829.

91. Yeo, D. S. Y.; Srinivasan, R.; Chen, G., Y. J.; Yao, S. Q. Chem. Eur. J. 2004, 10, 4664.

92. Leleu, S.; Penhoat, M.; Bouet, A.; Dupas, G.; Papamicäel, C.; Marsais, F.; Levacher, V. J. Am. Chem. Soc 2005, 127, 15668.

93. Canne, L. E.; Bark, S. J.; Kent, S. B. H. J. Am. Chem. Soc. 1996, 118, 5891.

94. Kang, J.; P., R. J.; Macmillan, D. Chem. Comm. 2009, 407.

95. Nagaike, F.; Onuma, Y.; Kanasawa, C.; Hojo, H.; Ueki, A.; Nakahara, Y.; Nakahara, Y. Org. Lett. 2006, 8, 4465.

96. Kimmerlin, T.; Seebach, D. J. Peptide Res. 2005, 65, 229.

97. Offer, J.; Dawson, P. E. Org. Lett. 2000, 2, 23.

98. Mao, H.; Hart, S. A.; Schink, A.; Pollok, B. A. J. Am. Chem. Soc. 2004, 126, 2670.

99. Saxon, E.; Armstrong, J. I.; Bertozzi, C. R. Organic Lett. 2000, 2, 2141.

100. Botti, P.; Carrasco, M. R.; Kent, B. H. Tetrahedron Lett. 2001, 42, 1831.

101. Wu, B.; Chen, J.; Warren, J. D.; G., C.; Hua, Z.; Danishefsky, S. J. Angew. Chem. Int. Ed. 2006, 45, 4116.

102. Bang, D.; Pentelupe, B. L.; Gates, Z. P.; Kent, S. B. Org. Lett. 2006, 8, 1049.

103. Wan, Q.; Chen, J.; Yuan, Y.; Danishefsky, S. J. J. Am. Chem. Soc. 2008, 130, 15814.

104. Warren, J. D.; Miller, J. S.; Keding, S. J.; Danishefsky, S. J. J. Am. Chem. Soc. 2004, $126,6576$.

105. Blanco-Canosa, J. B.; Dawson, P. E. Angew. Chem. Int. Ed. 2008, 47, 6851.

106. Chen, J.; Wan, Q.; Zhu, J.; Danishefsky, S. J. Angew. Chem. Int. Ed. 2008, 47, 8521.

107. Fukuzumi, T.; Bode, J. W. J. Am. Chem. Soc. 2009, 131, 3864.

108. Ju, L.; Bode, J. W. Org. Biol. Chem. 2009, 7, 2259.

109. Curtius, T. J. Prakt. Chem. 1881, 24, 239.

110. Fischer, E.; Fourneau, E. Ber. Dtsch. Chem. Ges. 1901, 34.

111. Macmillan, D. Angew. Chem. Int. Ed. 2006, 45, 7668. 